Copper-promoted Tandem Reaction of Azobenzenes with Allyl Bromides via $\mathrm{N}=\mathrm{N}$ Bond Cleavage for the Regioselective Synthesis of Quinolines

$$
\text { Xiangli } \mathrm{Yi}^{\mathrm{a}} \text {, Chanjuan } \mathrm{Xi}^{\mathrm{ab}}
$$

${ }^{\mathrm{a}}$ Key Laboratory of Bioorganic Phosphorus Chemistry \& Chemical Biology

(Ministry of Education), Department of Chemistry, Tsinghua University, Beijing

100084, China;

${ }^{\mathrm{b}}$ State Key Laboratory of Elemento-Organic Chemistry, Nankai University, Tianjin 300071, China

E-mail: cjxi@tsinghua.edu.cn

\title{
List of the contents:
}

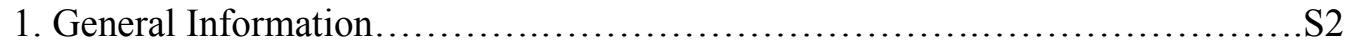

2. General procedures................................................. 3

3. Some experiment details for mechanism investigation.....................S6

4. Characterization data of products and some substrates......................S8

4. References........................................................... 19

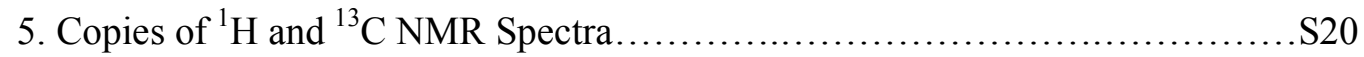




\section{General Information}

All the reactions were carried out in sealed tubes with Teflon-lined septum under $\mathrm{N}_{2}$ atmosphere. Chemicals were either purchased or synthesized according to relevant literatures and all reagents were used directly without further purification. Solvents including toluene and $\mathrm{CH}_{3} \mathrm{CN}$ were fresh distilled, while 1,2-dichloroethane (DCE) and $\mathrm{CHCl}_{3}$ were dried over activated $4 \AA$ molecular sieve. Gas Chromatography-Mass Spectrometer (GC-MS2010 SE) was used for reaction mixture analysis and product identification. Silica gel column chromatography was used for product separation (particle size 200-300 mesh). ${ }^{1} \mathrm{H}$ NMR and ${ }^{13} \mathrm{C}$ NMR spectra were recorded on $300 \mathrm{MHz}, 400 \mathrm{MHz}$ or $600 \mathrm{MHz}$ NMR spectrometer at ambient temperature with $\mathrm{CDCl}_{3}$ as the solvent. Chemical shifts $(\delta)$ were given in ppm, with the residual proton resonance of $\mathrm{CDCl}_{3}(7.26)$ and the carbon resonance of $\mathrm{CDCl}_{3}$ (77.16) as reference, respectively in ${ }^{1} \mathrm{H}$ - and ${ }^{13} \mathrm{C}-\mathrm{NMR}$ spectra. ${ }^{1} \mathrm{H}-\mathrm{NMR}$ yields were obtained using trichloroethylene as the internal standard $(9.00 \mu \mathrm{L}, 0.80$ mmol). All chemical yields are calculated based on the related azobenzenes $\mathbf{1}$. One molecule azobenzene $\mathbf{1}$ can form one molecule quinoline $\mathbf{3}$, because one equivalent $\mathbf{1}$ can only oxidize one equivalent $\mathrm{N}$-allylaniline $\mathbf{5}$ to generate 3 . When $\mathbf{1}$ acts only as oxidant and not construction unit, one equivalent 1 can form two equivalents of aniline 4 or $\mathrm{N}$-allylaniline 5 . 


\section{General procedures}

\subsection{Synthesis of azobenzenes 1}

Most of azobenzenes (1a-1m, 1o) were synthesized according to the literature reported. ${ }^{1}$ Aniline $(5 \mathrm{mmol}), \mathrm{CuBr}(0.3 \mathrm{mmol}, 43.2 \mathrm{mg})$ and toluene $(20 \mathrm{~mL}$, without drying) were added to a $50 \mathrm{~mL}$ flask. Then, pyridine $(0.9 \mathrm{mmol}, 73 \mu \mathrm{L})$ were added and the mixture were stirred at $60{ }^{\circ} \mathrm{C}$ for $20 \mathrm{~h}$, exposing to air in a fume cupboard. When the reaction completed, 2 gram silica gel were added to the mixture and the mixture were evaporated to give black powder. The products were obtained by eluting the powder over silica gel column (petroleum ether: ethyl acetate 25:1) and identified by ${ }^{1} \mathrm{H}-\mathrm{NMR}$.

Compound 1n was synthesized referring to the synthetic procedures of asymmetric azobenzene in literacture $2 .^{2}$

\subsection{Synthesis of substituted allyl bromide 2}

Allyl bromides $(\mathbf{2 b}, \mathbf{2 g}, \mathbf{2 h}$ and $\mathbf{2 i})$ were synthesized by bromination of the corresponding substituted allyl alcohol in a phase-vanishing reaction. ${ }^{3}$ The procedure is exemplified by the synthesis of $\mathbf{2} \mathbf{b}$. To a test tube were added $2 \mathrm{~mL}$ perflorohexane. Then, $\mathrm{PBr}_{3}$ (7 mmol, 1.05 equiv., $0.67 \mathrm{~mL}$ ) was added slowly with a syringe, sinking to the bottom to form two phases. The corresponding crotyl alcohol solution (20 mmol, dissolved in $10 \mathrm{~mL}$ diethyl ether) was added slowly above the perflorohexane, and a triphasic system was formed. The reaction was conducted at room temperature, carefully stirring in the bottom layer, for $20 \mathrm{~h}$. when the reaction completed, the upper layer was washed with aqueous sodium bicarbonate and then water. The organic phase was dried over $\mathrm{MgSO}_{4}$, and distilled to furnish the final product. Notably, 2g, 
$\mathbf{2 h}$ was obtained by distillation under reduced pressure, while $\mathbf{2 i}$ was obtained by direct evaporation to remove the solvent.

The corresponding allyl alcohols were purchased commercially except $\mathbf{2 g}$ and $\mathbf{2 h}$, which were prepared referring to literature., ${ }^{4,5}$

\subsection{Procedure for reaction of 1 with 2}

A sealed tube was charged with the mixture of $1(0.2 \mathrm{mmol}), \mathrm{CuI}(0.2 \mathrm{mmol}, 38.2$ $\mathrm{mg}), 2(0.4 \mathrm{mmol})$, then stirred in 1, 2-dichloroethane (DCE, $2 \mathrm{~mL})$ at given temperature under nitrogen atmosphere for a given reaction time. When the reaction completed, water $(2 \mathrm{~mL})$ and ammonia $(0.5 \mathrm{~mL}, 25 \%$ weight $)$ were added and sufficiently mixed with the organic phase. Then, the mixture was extracted with dichloromethane $(2 \mathrm{~mL} \times 3)$. After being dried over $\mathrm{MgSO}_{4}$, the organic phase was evaporated, and the final product 3 was obtained by eluting the residue over silica gel column [petroleum ether $(\mathrm{PE}) /$ ethyl acetate $(\mathrm{EA}) /$ triethylamine $\left.\left(\mathrm{NEt}_{3}\right)\right]$.

\subsection{Synthetic details in mechanism studies}

\subsubsection{Synthesis of hydrobromide of $\mathrm{N}$-allylaniline $\mathbf{6} \mathbf{f a}$}

$255 \mu \mathrm{L}(3.0 \mathrm{mmol})$ allylbromide was dropwisely added two a mixture of $0.56 \mathrm{~mL}$ aniline $(6.0 \mathrm{mmol})$ and $0.42 \mathrm{~g}$ potassium carbonate in $6 \mathrm{~mL}$ DMF. The reaction was conducted at $0{ }^{\circ} \mathrm{C}$ for 6 hours. Ethyl acetate $10 \mathrm{~mL}$ was added to reaction mixture and DMF was removed by washing with $5 \mathrm{~mL}$ water for 3 times. The residue obtained by 
evaporation of the organic phase was separated over a Silica gel column (PE:EA=50:1)

to afford N-allylaniline $\mathbf{5 f a}(0.165 \mathrm{~g}, 41 \%){ }^{6}$

$0.165 \mathrm{~g} \mathrm{~N}$-allylaniline was added to $5 \mathrm{~mL}$ diethyl ether, and then treated with a stream of $\mathrm{HBr}$ gas. White precipitate was formed, which was then filtrated to give the final product $6 \mathbf{f a}(0.162 \mathrm{~g}, 62 \%)$.

\subsubsection{Synthesis of hydrobromide of cinnamaldehyde anil 8ac}

$0.66 \mathrm{~g}$ cinnamaldehyde $(5.0 \mathrm{mmol})$ was dropwisely added two a mixture of $0.535 \mathrm{~g}$ (5.0 mmol) $p$-toluidine and $1.0 \mathrm{~g} \mathrm{MgSO}_{4}$ in $6 \mathrm{~mL}$ diethyl ether. The reaction was conducted at room temperature for 2 hours. The reaction mixture was heated to $30{ }^{\circ} \mathrm{C}$ and filtrated to remove $\mathrm{MgSO}_{4}$. Subsequent cooling to $0{ }^{\circ} \mathrm{C}$ gave yellow precipitate, which was filtrated and then washed twice with $2 \mathrm{~mL}$ cold diethyl ether. $0.70 \mathrm{~g}(63 \%)$ cinnamaldehyde anil 7ac was obtained.

$0.70 \mathrm{~g} 7 \mathbf{a c}$ was added to $10 \mathrm{~mL}$ diethyl ether, and then treated with a stream of $\mathrm{HBr}$ gas. After the reaction completed, diethyl ether was evaporated to give yellow solid, which was then washed twice with $3 \mathrm{~mL}$ dry toluene. $0.51 \mathrm{~g} \mathrm{(53 \% )} \mathrm{8ac} \mathrm{was} \mathrm{obtained.}$ 


\section{Some details for mechanism investigation}

\subsection{GC graph for reaction of $1 p$ with aqueous hydrogen bromide}
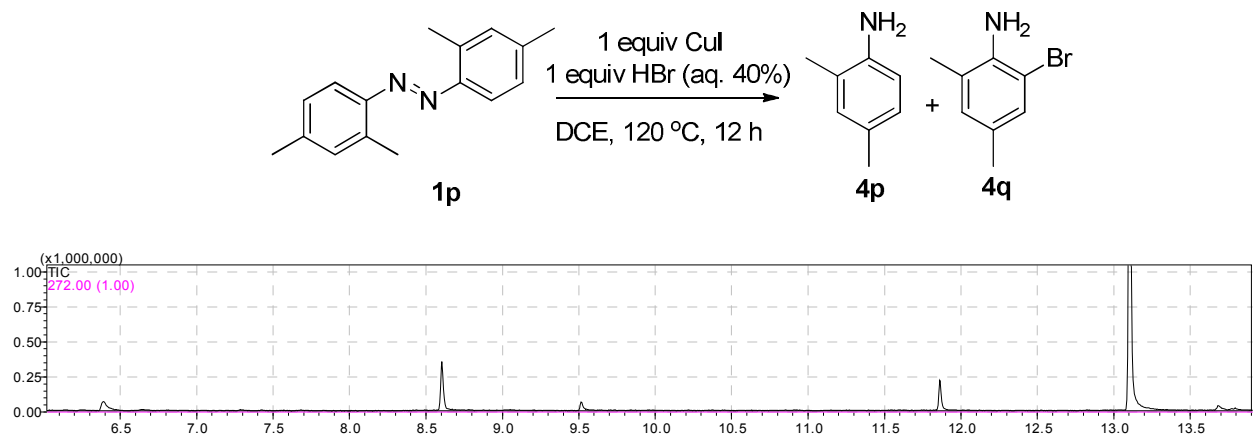

GC graph for reaction of $\mathbf{1 p}$ with aqueous hydrogen bromide

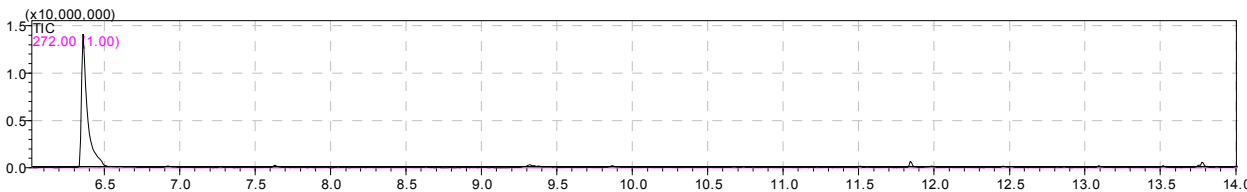

GC graph for $\mathbf{4 p}$

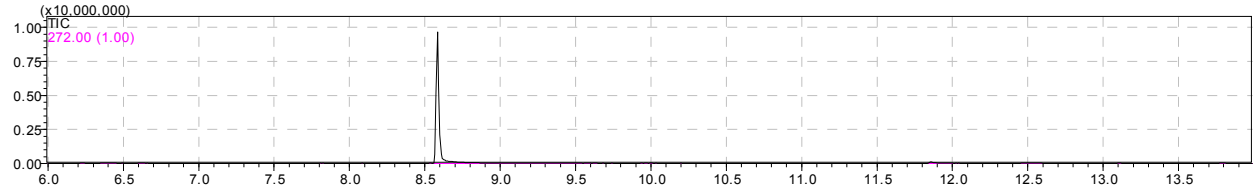

GC graph for $\mathbf{4 q}$

The GC graph for the reaction was compared to the GC graph of the pure substances $\mathbf{4 p}$ and $\mathbf{4 q}$ to verify the identity of the two products. The retention time and the MS graph both matched well (retention time: 1p 13.14 min; 4p 6.39 min; 4q 8.60 $\min )$. 


\subsection{Radical trap experiment}
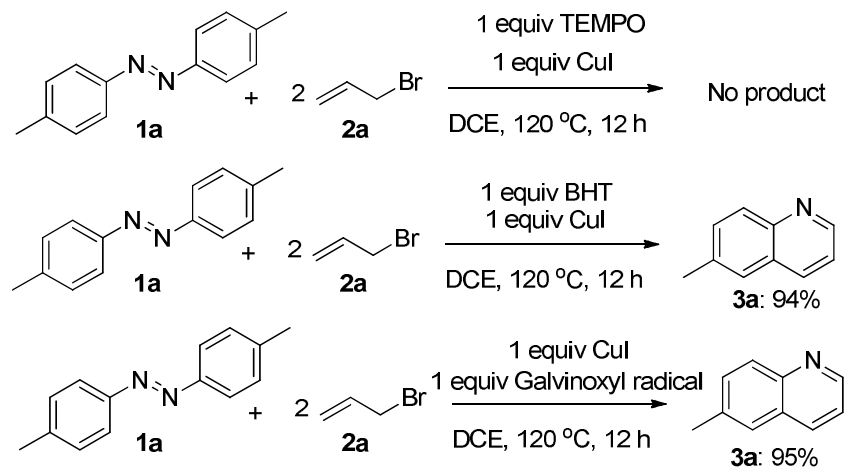

Radical trap experiment were conducted respectively with one equivalent of

TEMPO, BHT (butylated hydroxytoluene) and galvinoxyl radical. The first reagent completely inhibited the reaction while the latter two showed no impact. The

inhibition by TEMPO is not a reliable evidence for radical process because basic nitrogen atom in TEMPO may act as an inhibitor (addition of one equivalent of basic pyridine to our reaction could also result in a complete inhibition). However, we still believe the single electron process is possible and the failure of the latter two radical trap may have resulted from reactivity mismatch. 


\section{Characterization data of products and some substrates}<smiles>Cc1ccc2ncccc2c1</smiles>

6-Methylquinoline (3aa) ${ }^{7}$ : colorless oil, (23.4 mg, 82\% yield); ${ }^{1} \mathrm{H}$ NMR (400 MHz, $\left.\mathrm{CDCl}_{3}\right): \delta=8.85(\mathrm{~s}, 1 \mathrm{H}), 8.06(\mathrm{~d}, J=8.2 \mathrm{~Hz}, 1 \mathrm{H}), 8.00(\mathrm{~d}, J=8.5 \mathrm{~Hz}, 1 \mathrm{H}), 7.58-$ $7.52(\mathrm{~m}, 2 \mathrm{H}), 7.35(\mathrm{dd}, J=8.2,4.1 \mathrm{~Hz}, 1 \mathrm{H}), 2.53(\mathrm{~s}, 3 \mathrm{H}) ;{ }^{13} \mathrm{C}$ NMR $(100 \mathrm{MHz}$ $\left.\mathrm{CDCl}_{3}\right): \delta=149.6,147.0,136.5,135.4,131.8,129.2,128.4,126.7,121.2,21.7$; GCMS: 143.

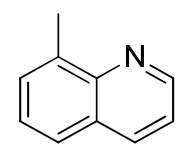

8-Methylquinoline (3ba) ${ }^{7}$ : colorless oil, (17.2 mg, 60\% yield); ${ }^{1} \mathrm{H}$ NMR (400 MHz, $\left.\mathrm{CDCl}_{3}\right): \delta=8.96(\mathrm{dd}, J=4.2,1.8 \mathrm{~Hz}, 1 \mathrm{H}), 8.14(\mathrm{dd}, J=8.2,1.8 \mathrm{~Hz}, 1 \mathrm{H}), 7.67(\mathrm{~d}, J=$ $8.2 \mathrm{~Hz}, 1 \mathrm{H}), 7.57(\mathrm{~d}, J=6.9 \mathrm{~Hz}, 1 \mathrm{H}), 7.48-7.38(\mathrm{~m}, 2 \mathrm{H}), 2.83(\mathrm{~s}, 3 \mathrm{H}) ;{ }^{13} \mathrm{C} \mathrm{NMR}$ $\left(100 \mathrm{MHz}, \mathrm{CDCl}_{3}\right): \delta=149.4,147.5,137.2,136.4,129.7,128.4,126.4,126.0,121.0$ 18.3; GC-MS: 143.

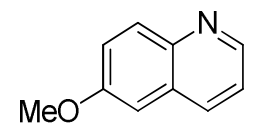

6-Methoxyquinoline (3ca) ${ }^{8}$ : colorless oil, $\left(18.8 \mathrm{mg}, 59 \%\right.$ yield); ${ }^{1} \mathrm{H}$ NMR (400 $\left.\mathrm{MHz}, \mathrm{CDCl}_{3}\right): \delta=8.76(\mathrm{~d}, J=3.4 \mathrm{~Hz}, 1 \mathrm{H}), 8.05(\mathrm{~d}, J=8.3 \mathrm{~Hz}, 1 \mathrm{H}), 8.01(\mathrm{~d}, J=9.2$ $\mathrm{Hz}, 1 \mathrm{H}), 7.39-7.34(\mathrm{~m}, 2 \mathrm{H}), 7.07(\mathrm{~d}, J=2.7 \mathrm{~Hz}, 1 \mathrm{H}), 3.93(\mathrm{~s}, 3 \mathrm{H}) ;{ }^{13} \mathrm{C}$ NMR $(100$ $\left.\mathrm{MHz}, \mathrm{CDCl}_{3}\right): \delta=157.9,148.0,144.4,135.0,130.9,129.5,122.5,121.5,105.3,55.7$

GC-MS: 159. 
$t$-Bu<smiles>[c]1ccc2cccnc2c1</smiles>

7-(tert-Butyl)quinoline (3da): yellowish oil, (33.9 mg, 92\% yield); Rf: 0.27 (PE: EA: $\left.\mathrm{NEt}_{3}=50: 2: 1\right) ;{ }^{1} \mathrm{H}$ NMR $\left(400 \mathrm{MHz}, \mathrm{CDCl}_{3}\right): \delta=8.88(\mathrm{~d}, J=3.7 \mathrm{~Hz}, 1 \mathrm{H}), 8.12$ $(\mathrm{d}, J=8.2 \mathrm{~Hz}, 1 \mathrm{H}), 8.08(\mathrm{~s}, 1 \mathrm{H}), 7.76(\mathrm{~d}, J=8.6 \mathrm{~Hz}, 1 \mathrm{H}), 7.65(\mathrm{~d}, J=8.6 \mathrm{~Hz}, 1 \mathrm{H})$, $7.35(\mathrm{dd}, J=8.2,4.3 \mathrm{~Hz}, 1 \mathrm{H}), 1.44(\mathrm{~s}, 9 \mathrm{H}) ;{ }^{13} \mathrm{C} \mathrm{NMR}\left(100 \mathrm{MHz}, \mathrm{CDCl}_{3}\right): \delta=152.8$, $150.4,148.6,135.6,127.3,126.4,125.6,124.8,120.6,35.2,31.2$; HRMS (ESI positive mode) calcd for $\mathrm{C}_{13} \mathrm{H}_{15} \mathrm{~N}+\mathrm{H}^{+}$186.1277, found 186.1272; GC-MS: 185 .

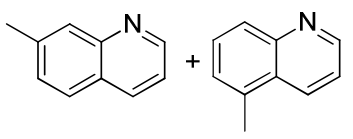

The mixture of 7-methylquinoline (3ea) ${ }^{9}$ and 5 -methylquinoline (3'ea) ${ }^{9}$ : colorless oil, (16.6 mg, 58\% yield, 1:0.20); ${ }^{1} \mathrm{H}$ NMR data for 3 ea $\left(400 \mathrm{MHz} \mathrm{CDCl}_{3}\right): \delta=8.87$ $(\mathrm{d}, J=3.5 \mathrm{~Hz}, 1 \mathrm{H}), 8.10(\mathrm{~d}, J=8.2 \mathrm{~Hz}, 1 \mathrm{H}), 7.88(\mathrm{~s}, 1 \mathrm{H}), 7.70(\mathrm{~d}, J=8.3 \mathrm{~Hz}, 1 \mathrm{H})$, $7.37(\mathrm{~d}, J=8.4 \mathrm{~Hz}, 1 \mathrm{H}), 7.32(\mathrm{dd}, J=8.1,4.2 \mathrm{~Hz}, 1 \mathrm{H}), 2.57(\mathrm{~s}, 3 \mathrm{H}) ; \mathrm{GC}-\mathrm{MS}: 143$ (two peaks).<smiles>c1ccc2ncccc2c1</smiles>

Quinoline (3fa) ${ }^{7}$ : colorless oil, (18.5 mg, 72\% yield); ${ }^{1} \mathrm{H}$ NMR (400 MHz, $\left.\mathrm{CDCl}_{3}\right): \delta=8.90(\mathrm{dd}, J=4.2,1.1 \mathrm{~Hz}, 1 \mathrm{H}), 8.13-8.09(\mathrm{~m}, 2 \mathrm{H}), 7.79(\mathrm{~d}, J=8.0 \mathrm{~Hz}$, 1H), $7.70(\mathrm{t}, J=7.7 \mathrm{~Hz}, 1 \mathrm{H}), 7.52$ (t, $J=7.1 \mathrm{~Hz}, 1 \mathrm{H}), 7.36(\mathrm{dd}, J=8.5,4.3 \mathrm{~Hz}, 1 \mathrm{H})$; ${ }^{13} \mathrm{C} \mathrm{NMR}\left(100 \mathrm{MHz}, \mathrm{CDCl}_{3}\right): \delta=150.5,148.4,136.2,129.6,128.4,127.9,126.7$, 121.2; GC-MS: 129.

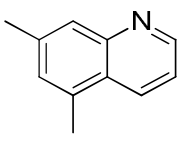


5,7-dimethylquinoline (3ga): yellowish oil, (22.5 mg, 66\% yield); Rf: 0.24 (PE:

EA: $\left.\mathrm{NEt}_{3}=50: 2: 1\right),{ }^{1} \mathrm{H}$ NMR $\left(600 \mathrm{MHz}, \mathrm{CDCl}_{3}\right): \delta=8.87(\mathrm{~s}, 1 \mathrm{H}), 8.26(\mathrm{~d}, J=8.4$ $\mathrm{Hz}, 1 \mathrm{H}), 7.73(\mathrm{~s}, 1 \mathrm{H}), 7.35(\mathrm{dd}, J=8.3,4.0 \mathrm{~Hz}, 1 \mathrm{H}), 7.21(\mathrm{~s}, 1 \mathrm{H}), 2.65(\mathrm{~s}, 3 \mathrm{H}), 2.52(\mathrm{~s}$, $3 \mathrm{H}) ;{ }^{13} \mathrm{C}$ NMR $\left(100 \mathrm{MHz}, \mathrm{CDCl}_{3}\right): \delta=150.0,148.9,139.4,134.2,132.3,129.4$, 126.7, 125.8, 120.0, 21.9, 18.6; HRMS (ESI positive mode) calcd for $\mathrm{C}_{11} \mathrm{H}_{11} \mathrm{~N}+\mathrm{H}^{+}$ 158.0964, found 158.0962; GC-MS: 157.

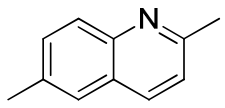

2,6-Dimethylquinoline (3ab) ${ }^{10}$ : colorless oil, (17.6 mg, 56\% yield); ${ }^{1} \mathrm{H}$ NMR (400 $\left.\mathrm{MHz}, \mathrm{CDCl}_{3}\right): \delta=7.94(\mathrm{~d}, J=8.4 \mathrm{~Hz}, 1 \mathrm{H}), 7.90(\mathrm{~d}, J=8.5 \mathrm{~Hz}, 1 \mathrm{H}), 7.54-7.46(\mathrm{~m}$, 2H), $7.23(\mathrm{~d}, J=8.4 \mathrm{~Hz}, 1 \mathrm{H}), 2.71(\mathrm{~s}, 3 \mathrm{H}), 2.50(\mathrm{~s}, 3 \mathrm{H}) ;{ }^{13} \mathrm{C} \mathrm{NMR}\left(100 \mathrm{MHz}, \mathrm{CDCl}_{3}\right)$ : $\delta=158.1,146.6,135.7,135.5,131.8,128.4,126.6,126.5,122.1,25.4,21.6 ;$ GC-MS: 157.

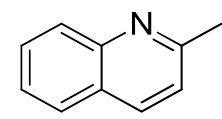

2-Methylquinoline (3fb) ${ }^{10}$ : colorless oil, (15.2 mg, 53\% yield); ${ }^{1} \mathrm{H}$ NMR (300 $\left.\mathrm{MHz}, \mathrm{CDCl}_{3}\right): \delta=8.06-8.00(\mathrm{~m}, 2 \mathrm{H}), 7.76(\mathrm{~d}, J=8.1 \mathrm{~Hz}, 1 \mathrm{H}), 7.70-7.64(\mathrm{~m}, 1 \mathrm{H})$, $7.51-7.44(\mathrm{~m}, 1 \mathrm{H}), 7.28(\mathrm{~d}, J=8.4 \mathrm{~Hz}, 1 \mathrm{H}), 2.75(\mathrm{~s}, 3 \mathrm{H}) ;{ }^{13} \mathrm{C}$ NMR $(100 \mathrm{MHz}$, $\left.\mathrm{CDCl}_{3}\right): \delta=159.1,148.0,136.3,129.5,128.7,127.6,126.6,125.8,122.1,25.5 ; \mathrm{GC}-$ MS: 143.<smiles>COc1ccc2ccc(C)nc2c1</smiles> 
7-Methoxy-2-methylquinoline (3hb) ${ }^{11}$ : yellowish oil, (9.6 mg, 28\% yield); ${ }^{1} \mathrm{H}$ NMR (400 MHz, $\left.\mathrm{CDCl}_{3}\right): \delta=7.94(\mathrm{~d}, J=8.3 \mathrm{~Hz}, 1 \mathrm{H}), 7.63(\mathrm{~d}, J=8.9 \mathrm{~Hz}, 1 \mathrm{H}), 7.35$ $(\mathrm{d}, J=2.5 \mathrm{~Hz}, 1 \mathrm{H}), 7.16-7.10(\mathrm{~m}, 2 \mathrm{H}), 3.93(\mathrm{~s}, 3 \mathrm{H}), 2.70(\mathrm{~s}, 3 \mathrm{H}) ;{ }^{13} \mathrm{C}$ NMR $(100$ $\left.\mathrm{MHz}, \mathrm{CDCl}_{3}\right): \delta=160.9,159.3,149.7,136.0,128.6,121.7,119.9,118.8,107.0,55.6$, 25.4; GC-MS:173.<smiles>Cc1ccc2cc(Cl)ccc2n1</smiles>

6-Chloro-2-methylquinoline (3ib) ${ }^{10}$ : white solid, (28.0 mg, 79\% yield); ${ }^{1} \mathrm{H}$ NMR $\left(400 \mathrm{MHz}, \mathrm{CDCl}_{3}\right): \delta=7.97-7.92(\mathrm{~m}, 2 \mathrm{H}), 7.74(\mathrm{~d}, J=1.8 \mathrm{~Hz}, 1 \mathrm{H}), 7.60(\mathrm{dd}, J=$ 9.1, $2.0 \mathrm{~Hz}, 1 \mathrm{H}), 7.29(\mathrm{~d}, J=8.4 \mathrm{~Hz}, 1 \mathrm{H}), 2.73(\mathrm{~s}, 3 \mathrm{H}) ;{ }^{13} \mathrm{C} \mathrm{NMR}\left(100 \mathrm{MHz}, \mathrm{CDCl}_{3}\right)$ : $\delta=159.5,146.4,135.4,131.4,130.4,127.2,126.3,123.0,25.5 ;$ GC-MS: 177<smiles>Cc1ccc2c(Cl)cccc2n1</smiles>

The mixture of 7- chloro-2-methylquinoline (3jb) 11 and 5-chloro-2methylquinoline (3'jb): white solid, (15.6 mg, 44\% yield, 1:0.24); ${ }^{1} \mathrm{H}$ NMR data for 3jb $\left(400 \mathrm{MHz}, \mathrm{CDCl}_{3}\right)$ : $\delta=7.99(\mathrm{~m}, 2 \mathrm{H}), 7.67(\mathrm{~d}, J=8.6 \mathrm{~Hz}, 1 \mathrm{H}), 7.40(\mathrm{dd}, J=8.7$, $2.1 \mathrm{~Hz}, 1 \mathrm{H}), 7.25$ (d, $J=8.2 \mathrm{~Hz}, 1 \mathrm{H}), 2.72$ (s, 3H); GC-MS: 177 (two peaks).<smiles>Cc1ccc2cc(F)ccc2n1</smiles>

6-Floro-2-methylquinoline (3kb) ${ }^{10}$ : white solid, (16.7 mg, 52\% yield); ${ }^{1} \mathrm{H}$ NMR 
$\left(400 \mathrm{MHz}, \mathrm{CDCl}_{3}\right): \delta=8.01-7.95(\mathrm{~m}, 2 \mathrm{H}), 7.43(\mathrm{t}, J=8.8 \mathrm{~Hz}, 1 \mathrm{H}), 7.36(\mathrm{~d}, J=8.8$ $\mathrm{Hz}, 1 \mathrm{H}), 7.28(\mathrm{~d}, J=8.2 \mathrm{~Hz}, 1 \mathrm{H}), 2.72(\mathrm{~s}, 3 \mathrm{H}) ;{ }^{13} \mathrm{C} \mathrm{NMR}\left(100 \mathrm{MHz}, \mathrm{CDCl}_{3}\right): \delta=$ $160.1(\mathrm{~d}, J=246.4 \mathrm{~Hz}), 158.4,145.0,135.6(\mathrm{~d}, J=5.2 \mathrm{~Hz}), 131.2(\mathrm{~d}, J=9.2 \mathrm{~Hz}), 127.1$ (d, $J=9.9 \mathrm{~Hz}), 122.8,119.5$ (d, $J=25.5 \mathrm{~Hz}), 110.6$ (d, $J=21.5 \mathrm{~Hz}), 25.3$; GC-MS: 161 .<smiles>Cc1ccc2cc(C(F)(F)F)ccc2n1</smiles>

2-Methyl-6-(trifloromethyl)quinoline (3lb) ${ }^{12}$ : white solid, (16.2 mg, 38\% yield);

${ }^{1} \mathrm{H}$ NMR $\left(400 \mathrm{MHz}, \mathrm{CDCl}_{3}\right): \delta=8.13-8.08(\mathrm{~m}, 3 \mathrm{H}), 7.85(\mathrm{dd}, J=8.8,2.0 \mathrm{~Hz}, 1 \mathrm{H})$, $7.39(\mathrm{~d}, J=8.5 \mathrm{~Hz}, 1 \mathrm{H}), 2.78(\mathrm{~s}, 3 \mathrm{H}) ;{ }^{13} \mathrm{C} \mathrm{NMR}\left(100 \mathrm{MHz}, \mathrm{CDCl}_{3}\right): \delta=161.7,149.0$, 136.9, 130.0, 127.8 (q, $J=32.6 \mathrm{~Hz}), 125.6,123.4,122.9,25.7$; GC-MS: 211.<smiles>Cc1ccc2ccc(C(F)(F)F)cc2n1</smiles>

2-Methyl-7-(trifloromethyl)quinoline $(\mathbf{3 m b})^{13}$ : white solid, (16.0 mg, 38\% yield); ${ }^{1} \mathrm{H}$ NMR $\left(400 \mathrm{MHz}, \mathrm{CDCl}_{3}\right): \delta=8.33(\mathrm{~s}, 1 \mathrm{H}), 8.08(\mathrm{~d}, J=8.5 \mathrm{~Hz}, 1 \mathrm{H}), 7.87(\mathrm{~d}, J=$ $8.5 \mathrm{~Hz}, 1 \mathrm{H}), 7.64(\mathrm{dd}, J=8.5,1.4 \mathrm{~Hz}, 1 \mathrm{H}), 7.39(\mathrm{~d}, J=8.4 \mathrm{~Hz}, 1 \mathrm{H}), 2.77(\mathrm{~s}, 3 \mathrm{H}) ;{ }^{13} \mathrm{C}$ NMR (76 MHz, $\left.\mathrm{CDCl}_{3}\right): \delta=160.8,147.0,136.0,131.2(\mathrm{q}, J=32.4 \mathrm{~Hz}), 128.7,128.1$, 126.7, 124.0, 121.4, 25.5; GC-MS:211.<smiles>Cc1ccc2nc(-c3ccccc3)ccc2c1</smiles>

6-Methyl-2-phenylquinoline (3ac) ${ }^{14}$ : white solid, (25.5 mg, 58\% yield); ${ }^{1} \mathrm{H}$ NMR $\left(400 \mathrm{MHz}, \mathrm{CDCl}_{3}\right): \delta=8.18-8.12(\mathrm{~m}, 3 \mathrm{H}), 8.07(\mathrm{~d}, J=8.5 \mathrm{~Hz}, 1 \mathrm{H}), 7.84(\mathrm{~d}, J=8.6$ $\mathrm{Hz}, 1 \mathrm{H}), 7.62-7.50(\mathrm{~m}, 4 \mathrm{H}), 7.48-7.43(\mathrm{~m}, 1 \mathrm{H}), 2.55(\mathrm{~s}, 3 \mathrm{H}) ;{ }^{13} \mathrm{C}$ NMR $(100 \mathrm{MHz}$, 
$\left.\mathrm{CDCl}_{3}\right): \delta=156.6,147.0,140.0,136.3,136.2,132.1,129.5,129.2,128.9,127.6,127.3$ 126.5, 119.1, 21.7; GC-MS:219.

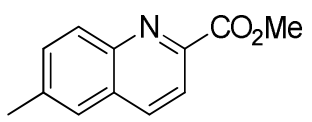

Methyl 6-methylquinoline-2-carboxylate (3ad) ${ }^{15}$ : white solid, $(29.0 \mathrm{mg}, 72 \%$ yield); ${ }^{1} \mathrm{H}$ NMR $\left(400 \mathrm{MHz}, \mathrm{CDCl}_{3}\right): \delta=8.23-8.11(\mathrm{~m}, 3 \mathrm{H}), 7.68-7.58(\mathrm{~m}, 2 \mathrm{H})$, $4.07(\mathrm{~s}, 3 \mathrm{H}), 2.56(\mathrm{~s}, 3 \mathrm{H}) ;{ }^{13} \mathrm{C} \mathrm{NMR}\left(100 \mathrm{MHz}, \mathrm{CDCl}_{3}\right): \delta=166.2,147.2,146.3$, 139.1, 136.6, 132.9, 130.5, 129.6, 126.5, 121.2, 53.3, 21.9; GC-MS:201.

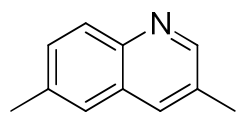

3,6-Dimethylquinoline (3ae) ${ }^{16}$ : yellowish oil, (15.6 mg, 49\% yield); ${ }^{1} \mathrm{H}$ NMR (300 $\left.\mathrm{MHz}, \mathrm{CDCl}_{3}\right): \delta=8.69(\mathrm{~d}, J=2.0 \mathrm{~Hz}, 1 \mathrm{H}), 7.95(\mathrm{~d}, J=8.3 \mathrm{~Hz}, 1 \mathrm{H}), 7.84-7.78(\mathrm{~m}$, $1 \mathrm{H}), 7.53-7.44(\mathrm{~m}, 2 \mathrm{H}), 2.52(\mathrm{~s}, 3 \mathrm{H}), 2.49(\mathrm{~s}, 3 \mathrm{H}) ;{ }^{13} \mathrm{C} \mathrm{NMR}\left(76 \mathrm{MHz}, \mathrm{CDCl}_{3}\right): \delta=$ 151.7, 145.3, 136.5, 134.2, 130.8, 130.5, 129.0, 128.3, 126.1, 21.7, 18.9; GC-MS:157.

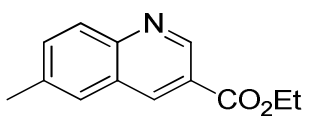

Ethyl 6-methylquinoline-3-carboxylate (3af): white solid, (30.8 mg, 72\% yield);

Mp: $55-57{ }^{\circ} \mathrm{C}$; Rf: 0.31 (PE: EA: $\left.\mathrm{NEt}_{3}=50: 2: 1\right) ;{ }^{1} \mathrm{H}$ NMR (400 MHz, $\left.\mathrm{CDCl}_{3}\right): \delta=$ $9.37(\mathrm{~d}, J=1.5 \mathrm{~Hz}, 1 \mathrm{H}), 8.74(\mathrm{~d}, J=1.8 \mathrm{~Hz}, 1 \mathrm{H}), 8.04$ (d, $J=8.6 \mathrm{~Hz}, 1 \mathrm{H}), 7.73-$ $7.62(\mathrm{~m}, 2 \mathrm{H}), 4.47(\mathrm{q}, J=7.1 \mathrm{~Hz}, 2 \mathrm{H}), 2.56(\mathrm{~s}, 3 \mathrm{H}), 1.45(\mathrm{t}, J=7.1 \mathrm{~Hz}, 3 \mathrm{H}) ;{ }^{13} \mathrm{C}$ NMR $\left(100 \mathrm{MHz}, \mathrm{CDCl}_{3}\right): \delta=165.7,149.4,148.6,138.1,137.6,134.2,129.2,128.0$, 127.1, 123.4, 61.6, 21.7, 14.5; HRMS (ESI positive mode) calcd for $\mathrm{C}_{13} \mathrm{H}_{13} \mathrm{NO}_{2}+\mathrm{H}^{+}$ 
216.1019, found 216.1015; GC-MS:215.

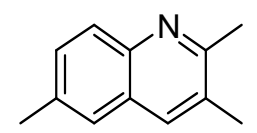

2,3,6-Trimethylquinoline (3ag): white solid, (14.3 mg, 42\% yield); $\mathrm{Mp}: 76-78{ }^{\circ} \mathrm{C}$;

Rf: 0.32 (PE: EA: $\left.\mathrm{NEt}_{3}=50: 2: 1\right) ;{ }^{1} \mathrm{H}$ NMR $\left(400 \mathrm{MHz}, \mathrm{CDCl}_{3}\right): \delta=7.87(\mathrm{~d}, J=8.5$ $\mathrm{Hz}, 1 \mathrm{H}), 7.71(\mathrm{~s}, 1 \mathrm{H}), 7.44-7.40(\mathrm{~m}, 2 \mathrm{H}), 2.64(\mathrm{~s}, 3 \mathrm{H}), 2.48(\mathrm{~s}, 3 \mathrm{H}), 2.40(\mathrm{~s}, 3 \mathrm{H}) ;{ }^{13} \mathrm{C}$ NMR (100 MHz, $\left.\mathrm{CDCl}_{3}\right): \delta=158.0,145.2,135.4,134.8,130.7,130.0,128.0,127.6$, 125.7, 23.5, 21.6, 19.7; HRMS (ESI positive mode) calcd for $\mathrm{C}_{12} \mathrm{H}_{13} \mathrm{~N}+\mathrm{H}^{+}$172.1121, found 172.1123; GC-MS:171.

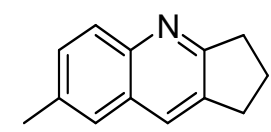

7-Methyl-2,3-dihydro-1H-cyclopenta[b]quinoline (3ah): white solid, (10.2 mg, 28\% yield); Mp: $65-68{ }^{\circ} \mathrm{C}$; Rf: 0.32 (PE: EA: $\left.\mathrm{NEt}_{3}=50: 2: 1\right) ;{ }^{1} \mathrm{H}$ NMR $(400 \mathrm{MHz}$, $\left.\mathrm{CDCl}_{3}\right): \delta=7.90(\mathrm{~d}, J=8.5 \mathrm{~Hz}, 1 \mathrm{H}), 7.79(\mathrm{~s}, 1 \mathrm{H}), 7.48(\mathrm{~s}, 1 \mathrm{H}), 7.44(\mathrm{dd}, J=8.5,1.9$ Hz, 1H), 3.14 (t, $J=7.6 \mathrm{~Hz}, 2 \mathrm{H}), 3.06$ (td, $J=7.5,1.4 \mathrm{~Hz}, 2 \mathrm{H}), 2.50(\mathrm{~s}, 3 \mathrm{H}), 2.19$ (p, $J=7.6 \mathrm{~Hz}, 2 \mathrm{H}) ;{ }^{13} \mathrm{C} \mathrm{NMR}\left(100 \mathrm{MHz}, \mathrm{CDCl}_{3}\right): \delta=167.1,146.2,135.7,135.3,130.7$, 129.9, 128.4, 127.6, 126.6, 34.7, 30.7, 23.8, 21.6; HRMS (ESI positive mode) calcd for $\mathrm{C}_{13} \mathrm{H}_{13} \mathrm{~N}+\mathrm{H}^{+}$184.1121, found 184.1122; GC-MS:183.<smiles>C=CCNc1c(C)cc(C)cc1C</smiles>

N-allyl-2,4,6-trimethylaniline (5oa): liquid, (7.6 mg, 11\% yield, isolated from 
reaction in eq. 3$) ;{ }^{1} \mathrm{H}$ NMR $\left(400 \mathrm{MHz}, \mathrm{CDCl}_{3}\right): \delta=6.84(\mathrm{~s}, 2 \mathrm{H}), 6.06-5.95(\mathrm{~m}, 1 \mathrm{H})$, $5.28(\mathrm{dq}, J=17.1,1.6 \mathrm{~Hz}, 1 \mathrm{H}), 5.13(\mathrm{ddt}, J=10.2,1.6,1.3 \mathrm{~Hz}, 1 \mathrm{H}), 3.57(\mathrm{~d}, J=6.1$ $\mathrm{Hz}, 2 \mathrm{H}), 2.28(\mathrm{~s}, 6 \mathrm{H}), 2.24(\mathrm{~s}, 3 \mathrm{H}) ;{ }^{13} \mathrm{C} \mathrm{NMR}\left(100 \mathrm{MHz}, \mathrm{CDCl}_{3}\right): \delta=143.1,136.7$, 131.7, 130.0, 129.6, 116.2, 51.7, 20.7, 18.5; GC-MS:175.

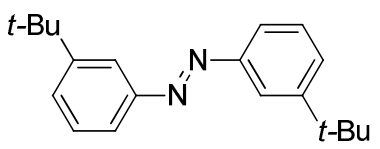

1,2-Bis(3-tert-butylphenyl)diazene (1d): yellow solid, (0.365 g, 50\% yield); ${ }^{1} \mathrm{H}$ NMR (400 MHz, $\left.\mathrm{CDCl}_{3}\right): \delta=7.98(\mathrm{~s}, 2 \mathrm{H}), 7.75-7.70(\mathrm{~m}, 2 \mathrm{H}), 7.55-7.50(\mathrm{~m}, 2 \mathrm{H})$, $7.44(\mathrm{t}, J=7.8 \mathrm{~Hz}, 2 \mathrm{H}), 1.41(\mathrm{~s}, 18 \mathrm{H})$.

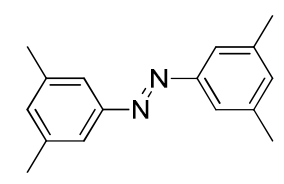

1,2-Bis(3,5-dimethylphenyl)diazene (1g): yellow solid, (0.449 g, 75\% yield); ${ }^{1} \mathrm{H}$ NMR (300 MHz, $\left.\mathrm{CDCl}_{3}\right): \delta=7.53(\mathrm{~s}, 4 \mathrm{H}), 7.12(\mathrm{~s}, 2 \mathrm{H}), 2.42(\mathrm{~s}, 12 \mathrm{H})$.

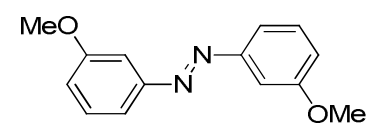

1,2-Bis(3-methoxyphenyl)diazene (1h): yellow solid, (0.314 g, 52\% yield); ${ }^{1} \mathrm{H}$ NMR $\left(300 \mathrm{MHz}, \mathrm{CDCl}_{3}\right): \delta=7.60-7.55(\mathrm{~m}, 2 \mathrm{H}), 7.48-7.40(\mathrm{~m}, 4 \mathrm{H}), 7.05(\mathrm{dd}, J=$ 8.1, $2.6 \mathrm{~Hz}, 2 \mathrm{H}), 3.91$ (s, 6H).

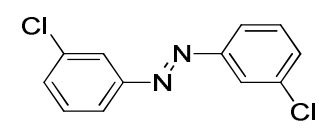

1,2-Bis(3-chlorophenyl)diazene (1j): yellow solid, (0.504 g, 80\% yield); ${ }^{1} \mathrm{H}$ NMR $\left(300 \mathrm{MHz}, \mathrm{CDCl}_{3}\right): \delta=7.92-7.89(\mathrm{~m}, 2 \mathrm{H}), 7.87-7.81(\mathrm{~m}, 2 \mathrm{H}), 7.55-7.40(\mathrm{~m}, 4 \mathrm{H})$. 


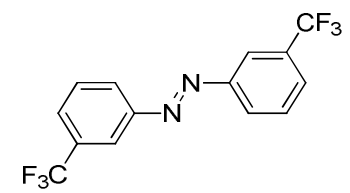

1,2-Bis((3-trifloromethyl)phenyl)diazene (1m): red solid, $\left(0.527 \mathrm{~g}, 66 \%\right.$ yield); ${ }^{1} \mathrm{H}$ NMR (300 MHz, $\left.\mathrm{CDCl}_{3}\right): \delta=8.22(\mathrm{~s}, 2 \mathrm{H}), 8.14(\mathrm{~d}, J=8.0 \mathrm{~Hz}, 2 \mathrm{H}), 7.77(\mathrm{~d}, J=7.7$ $\mathrm{Hz}, 2 \mathrm{H}), 7.67(\mathrm{t}, J=7.9 \mathrm{~Hz}, 2 \mathrm{H})$.

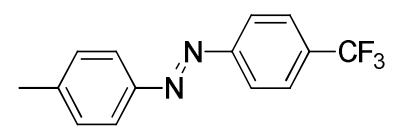

1-(p-Tolyl)-2-(4-(trifluoromethyl)phenyl)diazene (1n): yellow solid, (64 mg, 24\% yield); ${ }^{1} \mathrm{H}$ NMR $\left(400 \mathrm{MHz}, \mathrm{CDCl}_{3}\right): \delta=7.98(\mathrm{~d}, J=8.2 \mathrm{~Hz}, 2 \mathrm{H}), 7.86(\mathrm{~d}, J=8.3 \mathrm{~Hz}$, 2H), $7.77(\mathrm{~d}, J=8.5 \mathrm{~Hz}, 2 \mathrm{H}), 7.34(\mathrm{~d}, J=8.2 \mathrm{~Hz}, 2 \mathrm{H}), 2.46(\mathrm{~s}, 3 \mathrm{H})$.

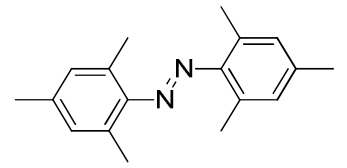

(E)-1,2-dimesityldiazene (10): red solid, (0.350 g, 53\% yield); ${ }^{1} \mathrm{H}$ NMR (400 MHz, $\left.\mathrm{CDCl}_{3}\right): \delta=6.98(\mathrm{~s}, 4 \mathrm{H}), 2.43(\mathrm{~s}, 12 \mathrm{H}), 2.35(\mathrm{~s}, 6 \mathrm{H})$.<smiles>C/C=C/CBr</smiles>

1-Bromobut-2-ene (2b): colorless liquid, (1.347 g, 51\% yield); ${ }^{1} \mathrm{H}$ NMR (400 MHz, $\left.\mathrm{CDCl}_{3}\right): \delta=5.88-5.66(\mathrm{~m}, 2 \mathrm{H}), 3.94(\mathrm{~d}, J=7.2 \mathrm{~Hz}, 2 \mathrm{H}), 1.73(\mathrm{~d}, J=6.1 \mathrm{~Hz}, 3 \mathrm{H})$.<smiles>CC=C(C)CBr</smiles>

(E)-1-Bromo-2-methylbut-2-ene (2g): colorless liquid, (0.852 g, 53\% yield); ${ }^{1} \mathrm{H}$ NMR (400 MHz, $\left.\mathrm{CDCl}_{3}\right): \delta=5.73-5.65(\mathrm{~m}, 1 \mathrm{H}), 3.98(\mathrm{~s}, 2 \mathrm{H}), 1.75(\mathrm{~s}, 3 \mathrm{H}), 1.63(\mathrm{~d}$, 
$J=6.8 \mathrm{~Hz}, 3 \mathrm{H})$.

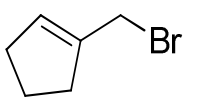

1-(Bromomethyl)cyclopent-1-ene (2h): colorless liquid, (1.503 g, 52\% yield); ${ }^{1} \mathrm{H}$

NMR (400 MHz, $\left.\mathrm{CDCl}_{3}\right): \delta=5.79(\mathrm{~s}, 1 \mathrm{H}), 4.08(\mathrm{~s}, 2 \mathrm{H}), 2.45-2.39(\mathrm{~m}, 2 \mathrm{H}), 2.39-$

$2.34(\mathrm{~m}, 2 \mathrm{H}), 1.99-1.91(\mathrm{~m}, 2 \mathrm{H})$.

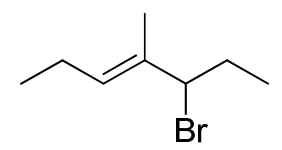

(E)-5-Bromo-4-methylhept-3-ene (2i): colorless liquid, (0.331 g, 58\% yield); ${ }^{1} \mathrm{H}$

NMR (400 MHz, $\left.\mathrm{CDCl}_{3}\right): \delta=5.86(\mathrm{t}, J=7.0 \mathrm{~Hz}, 1 \mathrm{H}), 4.83(\mathrm{t}, J=7.5 \mathrm{~Hz}, 1 \mathrm{H}), 2.42-$ $2.29(\mathrm{~m}, 3 \mathrm{H}), 2.27-2.16(\mathrm{~m}, 1 \mathrm{H}), 2.04(\mathrm{~s}, 3 \mathrm{H}), 1.32-1.21(\mathrm{~m}, 6 \mathrm{H})$.<smiles>C=CCNc1ccccc1</smiles>

N-allylaniline (5fa): colorless liquid, (0.165 g, 41\% yield); ${ }^{1} \mathrm{H}$ NMR (400 MHz, $\left.\mathrm{CDCl}_{3}\right): \delta=7.22-7.14(\mathrm{~m}, 2 \mathrm{H}), 6.74-6.68(\mathrm{~m}, 1 \mathrm{H}), 6.65-6.60(\mathrm{~m}, 2 \mathrm{H}), 5.96(\mathrm{ddt}, J$ $=17.0,10.4,5.3 \mathrm{~Hz}, 1 \mathrm{H}), 5.29(\mathrm{dq}, J=17.0,1.5 \mathrm{~Hz}, 1 \mathrm{H}), 5.17(\mathrm{dq}, J=10.1,1.4 \mathrm{~Hz}$ $1 \mathrm{H}), 3.78(\mathrm{~d}, J=5.4 \mathrm{~Hz}, 2 \mathrm{H})$.<smiles>C=CCNc1ccccc1Br</smiles>

Hydrobromide of $\mathrm{N}$-allylaniline (6fa): white solid, (0.162 g, 62\% yield); ${ }^{1} \mathrm{H}$ NMR $\left(400 \mathrm{MHz}, \mathrm{CDCl}_{3}\right): \delta=11.65(\mathrm{~s}, 2 \mathrm{H}), 7.60-7.56(\mathrm{~m}, 2 \mathrm{H}), 7.44-7.37(\mathrm{~m}, 3 \mathrm{H}), 6.05$ (ddt, $J=17.2,10.3,7.1 \mathrm{~Hz}, 1 \mathrm{H}), 5.34-5.24(\mathrm{~m}, 2 \mathrm{H}), 3.89(\mathrm{~d}, J=7.1 \mathrm{~Hz}, 2 \mathrm{H})$. 


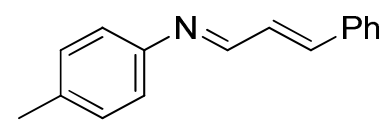

cinnamaldehyde anil (7ac): yellowish crystal, (0.70 g, 63\% yield); ${ }^{1} \mathrm{H}$ NMR (400

$\left.\mathrm{MHz}, \mathrm{CDCl}_{3}\right): \delta=8.28(\mathrm{dd}, J=5.4,2.6 \mathrm{~Hz}, 1 \mathrm{H}), 7.53(\mathrm{~d}, J=7.8 \mathrm{~Hz}, 2 \mathrm{H}), 7.42-7.31$

(m, 3H), $7.18(\mathrm{~d}, J=8.2 \mathrm{~Hz}, 2 \mathrm{H}), 7.14-7.09(\mathrm{~m}, 4 \mathrm{H}), 2.36(\mathrm{~s}, 3 \mathrm{H})$.

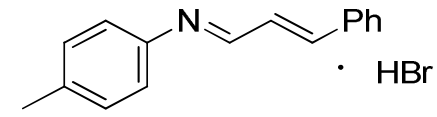

Hydrochloride of cinnamaldehyde anil (8ac): yellow solid, (0.51 g, 53\% yield);

${ }^{1} \mathrm{H}$ NMR $\left(400 \mathrm{MHz}, \mathrm{CDCl}_{3}\right): \delta=8.84(\mathrm{~d}, J=10.1 \mathrm{~Hz}, 1 \mathrm{H}), 8.01(\mathrm{dd}, J=15.6,10.0$

$\mathrm{Hz}, 1 \mathrm{H}), 7.79(\mathrm{~d}, J=15.6 \mathrm{~Hz}, 1 \mathrm{H}), 7.65(\mathrm{~d}, J=8.3 \mathrm{~Hz}, 2 \mathrm{H}), 7.56(\mathrm{~d}, J=8.1 \mathrm{~Hz}, 2 \mathrm{H})$,

$7.49-7.39(\mathrm{~m}, 1 \mathrm{H}), 7.35(\mathrm{t}, J=7.5 \mathrm{~Hz}, 2 \mathrm{H}), 7.11(\mathrm{~d}, J=8.2 \mathrm{~Hz}, 2 \mathrm{H}), 2.26(\mathrm{~s}, 3 \mathrm{H})$.<smiles>C/C=C(\C)CO</smiles>

(E)-2-Methylbut-2-en-1-ol (11g): colorless liquid, (0.920 g, 54\% yield); ${ }^{1} \mathrm{H}$ NMR

$\left(400 \mathrm{MHz}, \mathrm{CDCl}_{3}\right): \delta=5.57-5.44(\mathrm{~m}, 1 \mathrm{H}), 3.99(\mathrm{~s}, 2 \mathrm{H}), 1.66(\mathrm{~s}, 3 \mathrm{H}), 1.62(\mathrm{~d}, J=$ $6.7 \mathrm{~Hz}, 3 \mathrm{H}), 1.46(\mathrm{~s}, 1 \mathrm{H})$.<smiles>OCC1=CCCC1</smiles>

Cyclopent-1-en-1-ylmethanol (11h): colorless liquid, (1.765 g, 90\% yield); ${ }^{1} \mathrm{H}$ NMR (400 MHz, $\left.\mathrm{CDCl}_{3}\right): \delta=5.62-5.58(\mathrm{~m}, 1 \mathrm{H}), 4.17(\mathrm{~s}, 2 \mathrm{H}), 2.38-2.28(\mathrm{~m}, 4 \mathrm{H})$, $1.98-1.85(\mathrm{~m}, 2 \mathrm{H}), 1.61(\mathrm{~s}, 1 \mathrm{H})$. 


\section{References}

(1) Zhang, C.; Jiao, N. Angew. Chem. Int. Ed. 2010, 49, 6174.

(2) Takeda, Y.; Okumura, S.; Minakata, S. Angew. Chem. Int. Ed. 2012, 51, 7804.

(3) Jana, N. K.; Verkade, J. G. Org. Lett. 2003, 5, 3787.

(4) Fotiadou, A. D.; Zografos, A. L. Org. Lett. 2011, 13, 4592.

(5) Kapat, A.; Nyfeler, E.; Giuffredi, G. T.; Renaud, P. J. Am. Chem. Soc. 2009, 131, 17746.

(6) Fang, X.; Liu, K.; Li, C. J. Am. Chem. Soc. 2010, 132, 2274

(7) Chakraborty, S.; Brennessel, W. W.; Jones, W. D. J. Am. Chem. Soc. 2014, 136, 8564.

(8) Shakya, N.; Roy, K. K.; Saxena, A. K. Bioorgan Med. Chem. 2009, 17, 830.

(9) Shields, J. D.; Ahneman, D. T.; A. Graham, T. J.; Doyle, A. G. Org. Lett. 2014, 16, 142.

(10) Wu, J.; Talwar, D.; Johnston, S.; Yan, M.; Xiao, J. Angew. Chem. Int. Ed. 2013, 52,6983 .

(11) Matsubara, Y.; Hirakawa, S.; Yamaguchi, Y.; Yoshida, Z. Angew. Chem. Int. Ed. 2011, 50, 37670 .

(12) Chandrashekarappa, K. K. H.; Mahadevan, K. M.; Manjappa, K. B. Tetrahedron Lett. 2013, 54, 1368.

(13) Polanski, J.; Zouhiri, F.; Jeanson, L.; Desmaële, D.; d'Angelo, J.; Mouscadet, J.F.; Gieleciak, R.; Gasteiger, J.; Bret, M. L. J. Med. Chem. 2002, 45, 4647.

(14) Gómez, C. M. M.; Kouznetsov, V. V.; Sortino, M. A.; Álvarez, S. L.; Zacchino, S. A. Bioorgan. Med. Chem. 2008, 16, 7908.

(15) Zheng, Q.; Wang, S.; Liu, W. Tetrahedron 2014, 70, 7686.

(16) Monrad, R. N.; Madsen, R. Org. Biomol. Chem. 2011, 9, 610. 
6. Copies of ${ }^{1} \mathrm{H}$ and ${ }^{13} \mathrm{C}$ NMR Spectra

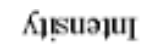

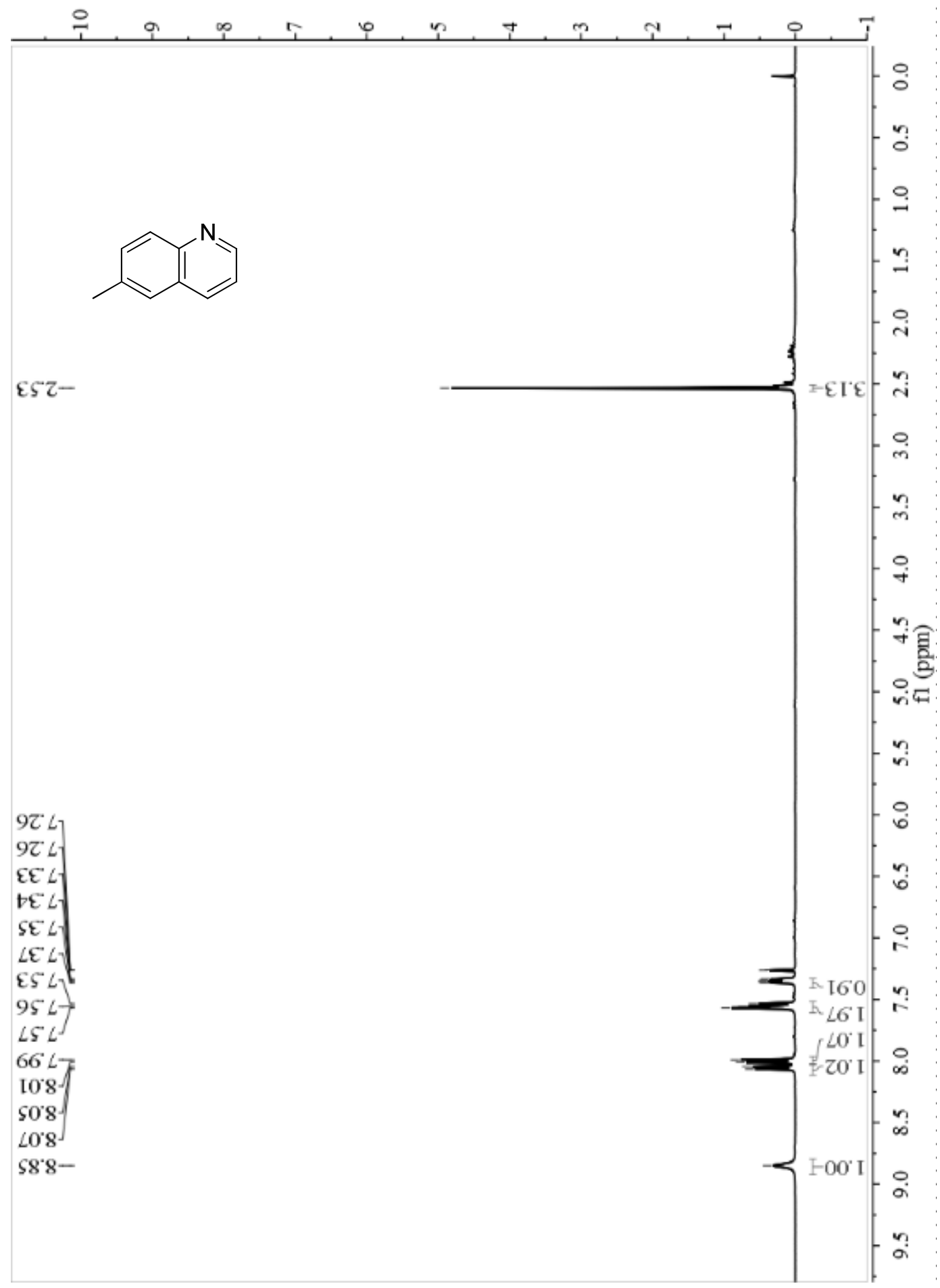

${ }^{1} \mathrm{H}$ NMR of Compound $\mathbf{3 a a}$ 


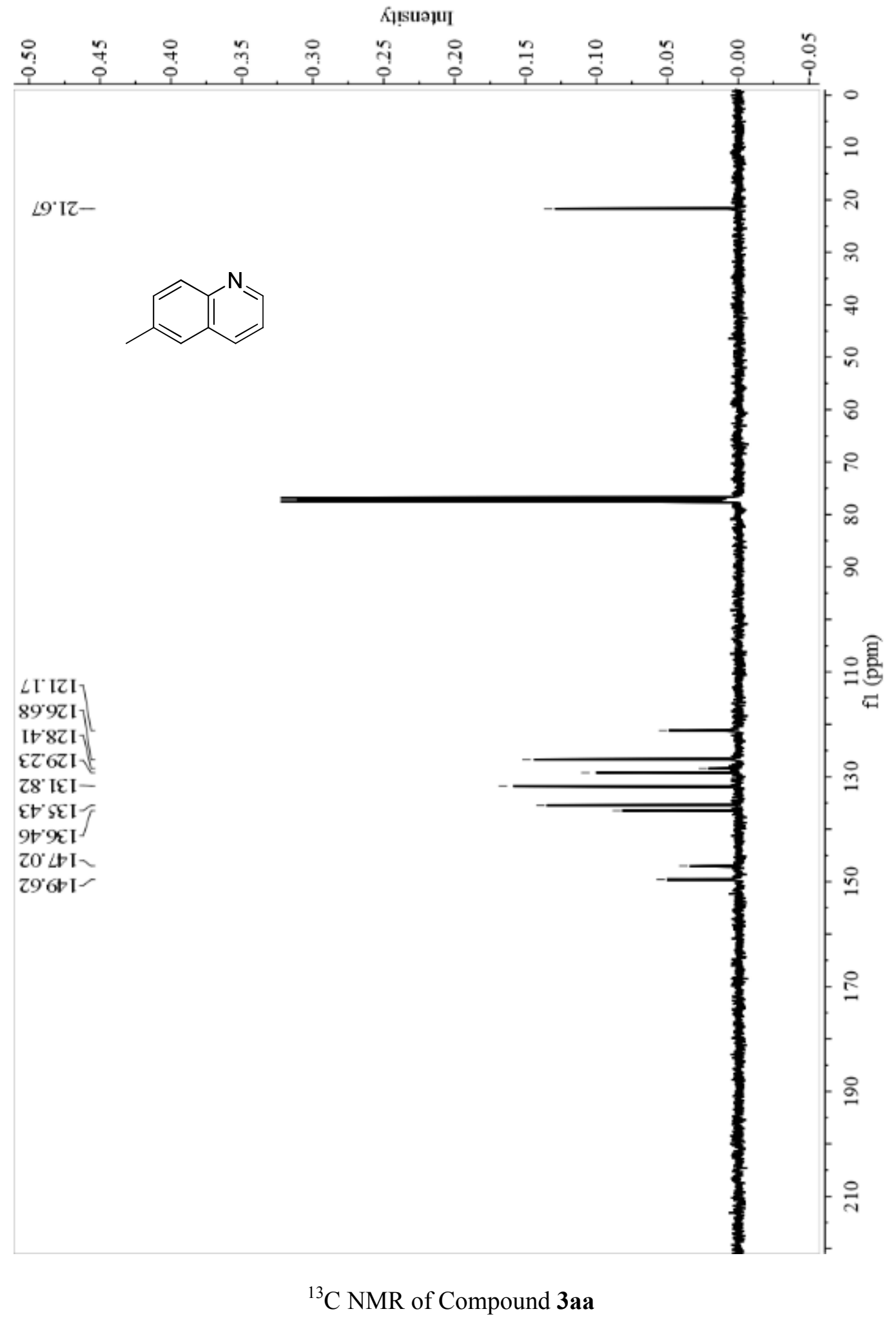




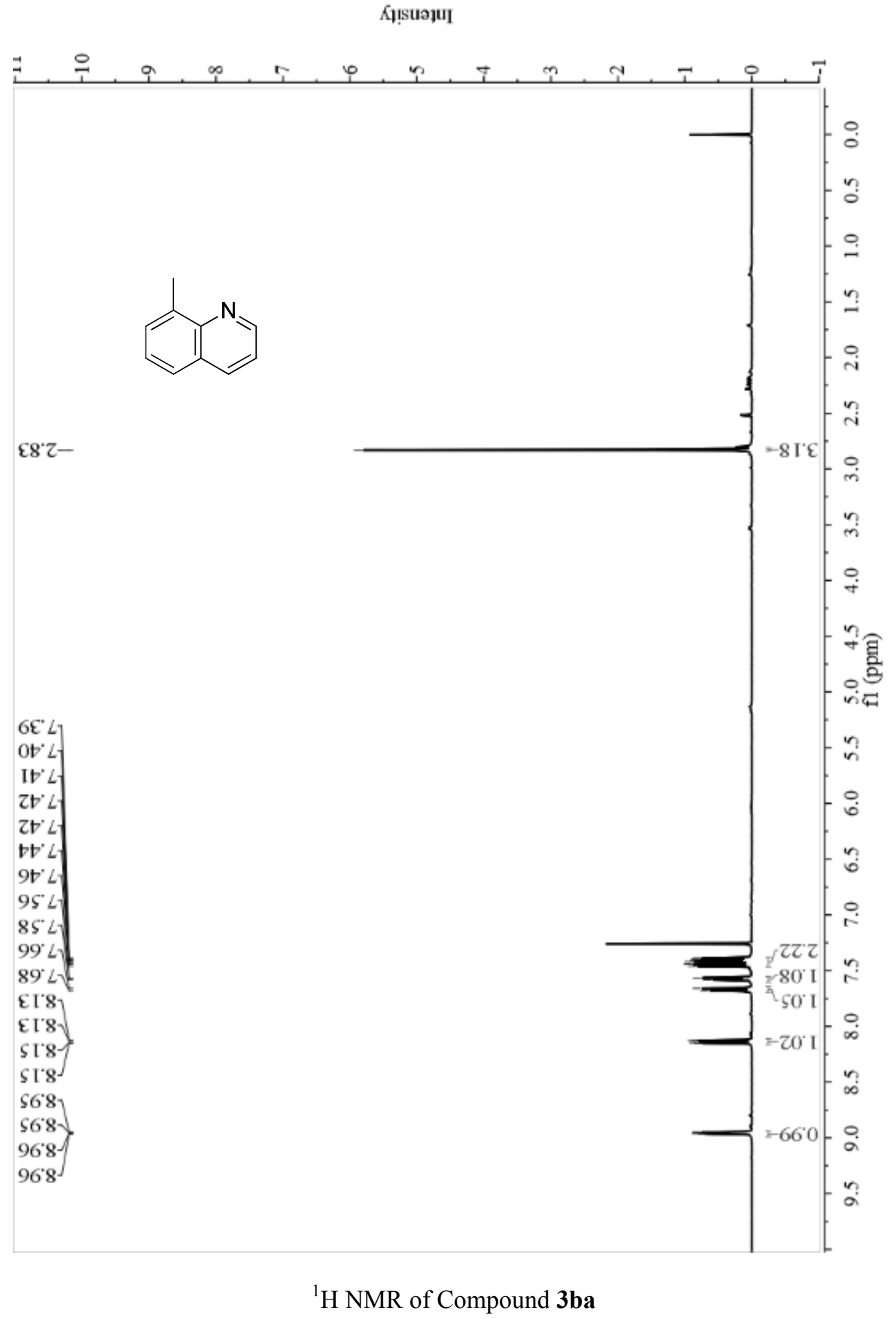




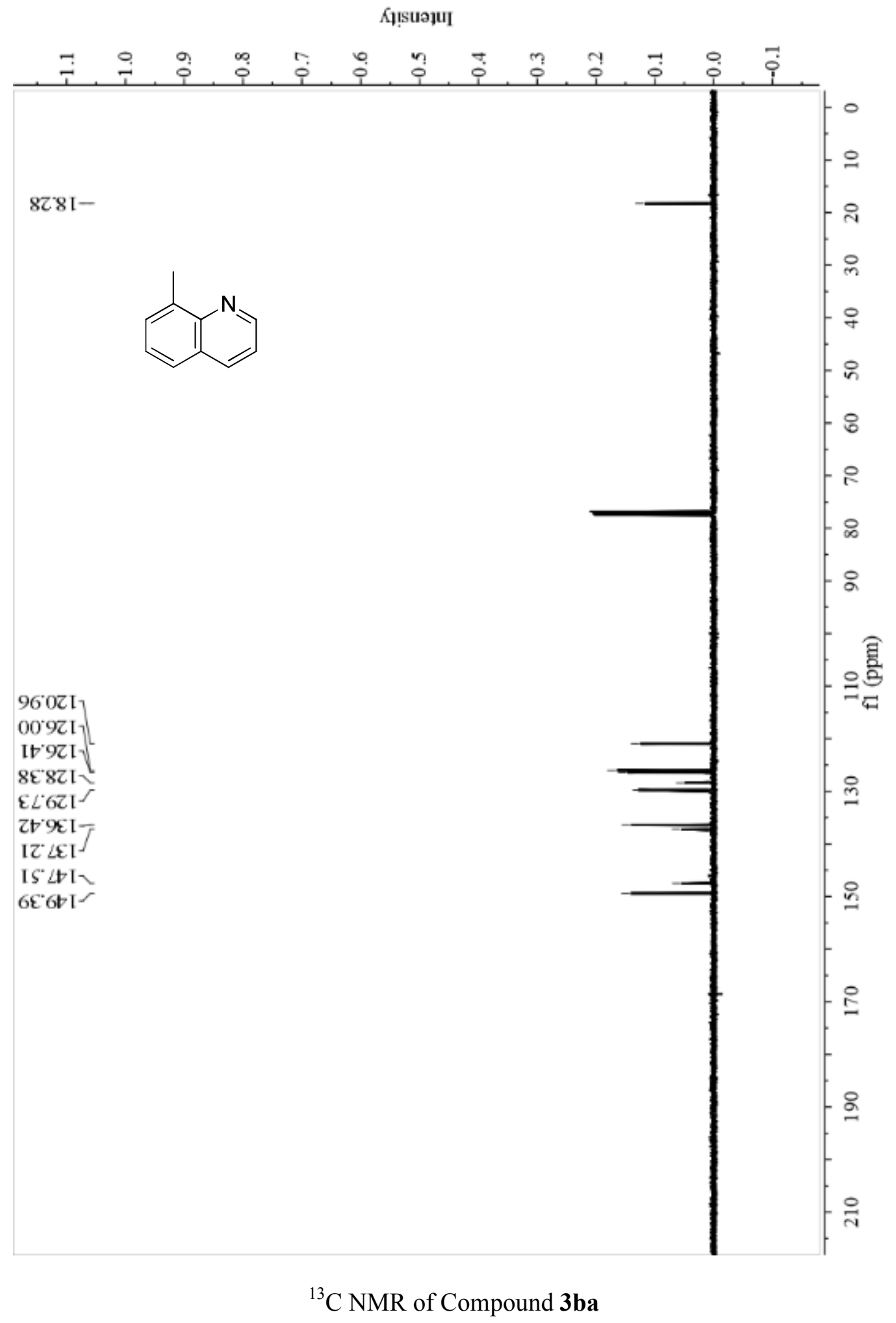




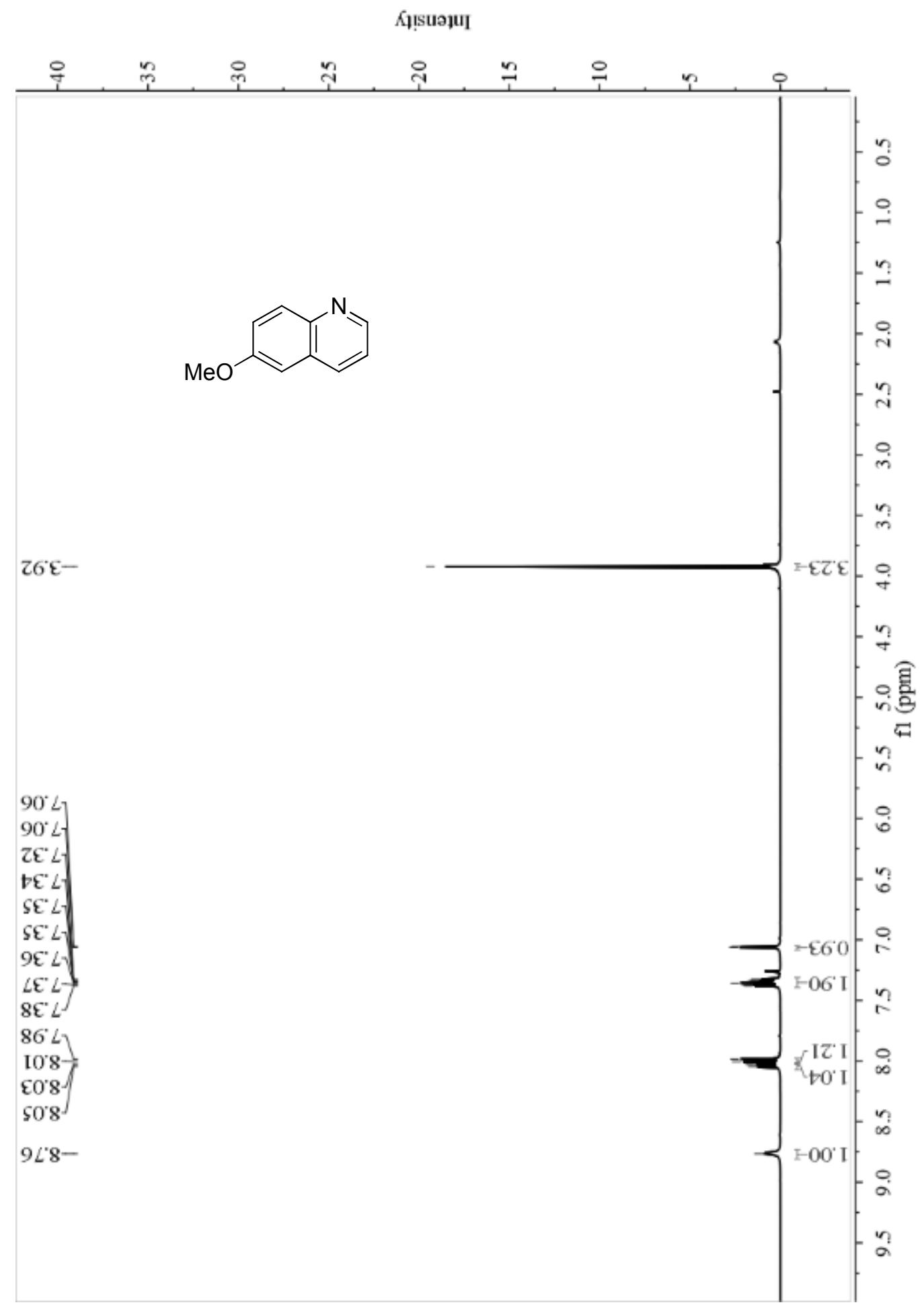

${ }^{1} \mathrm{H}$ NMR of Compound 3ca 


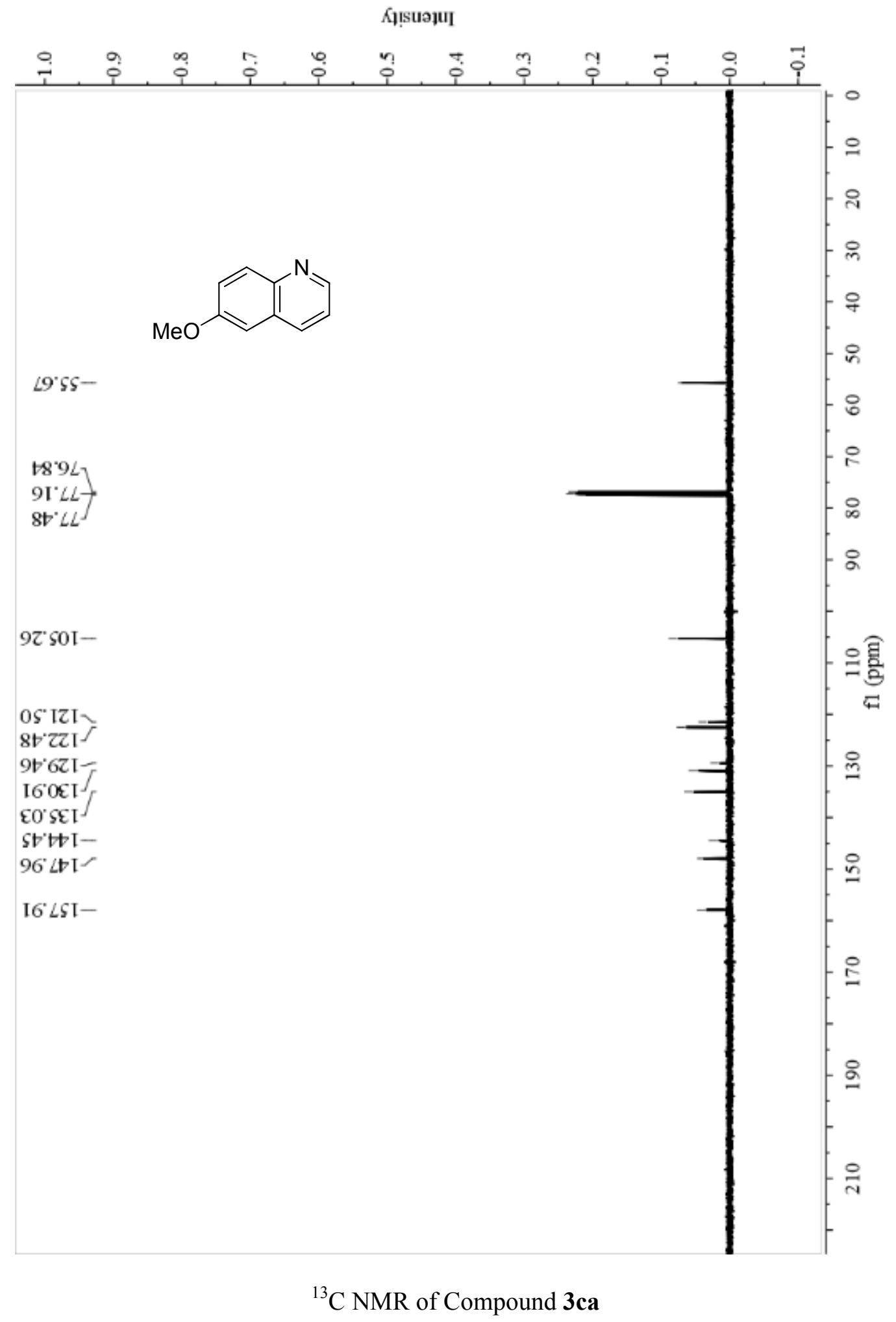




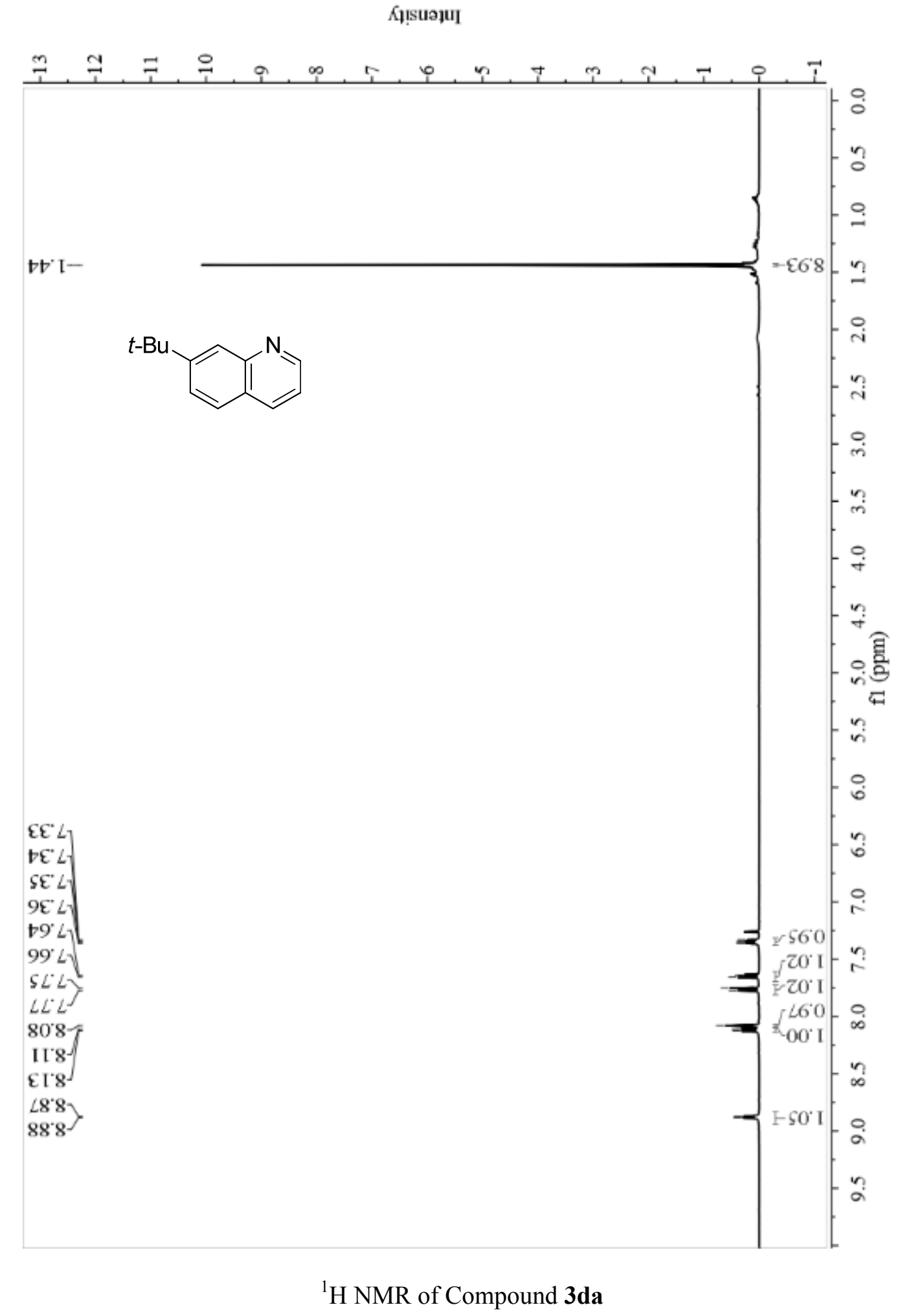




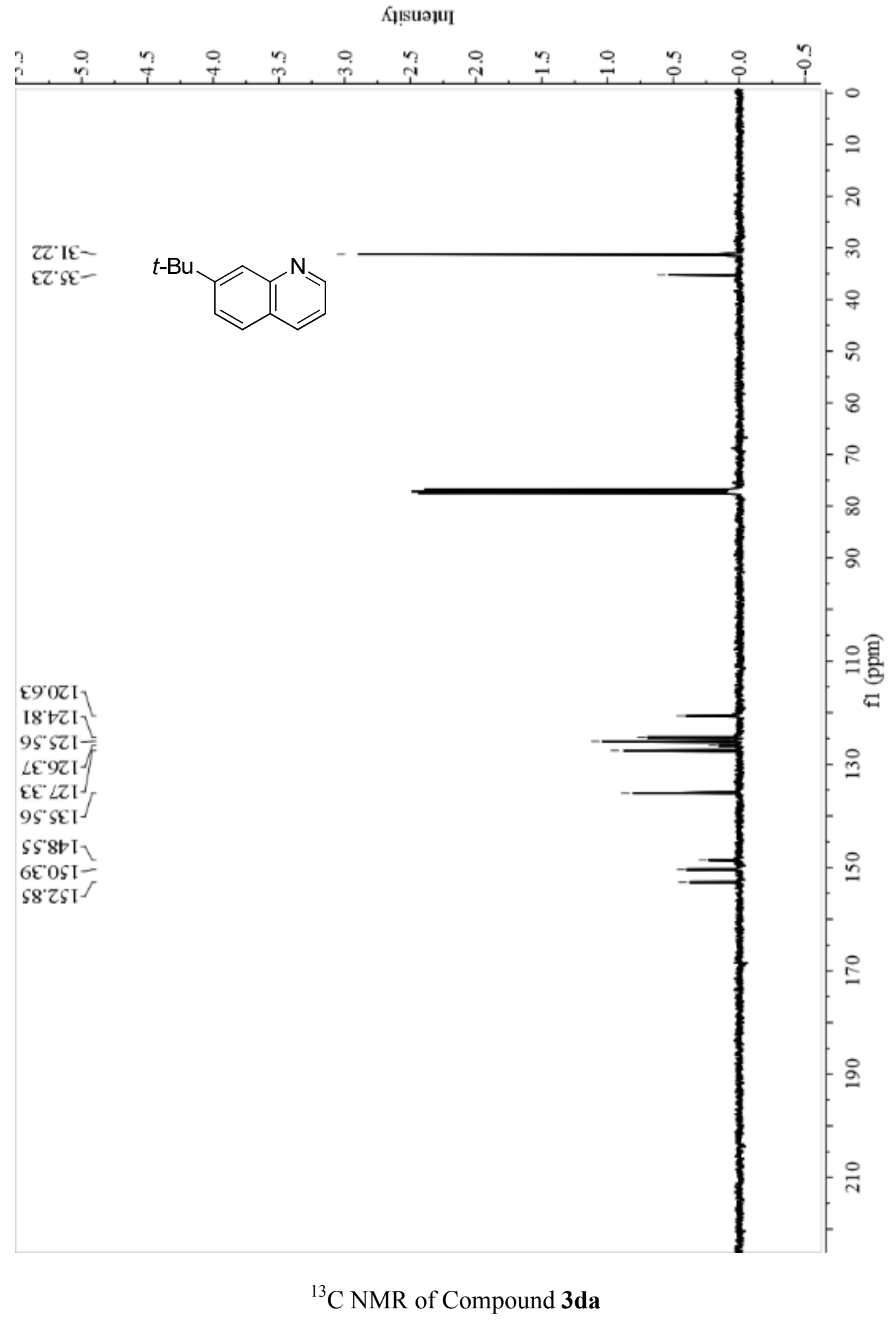




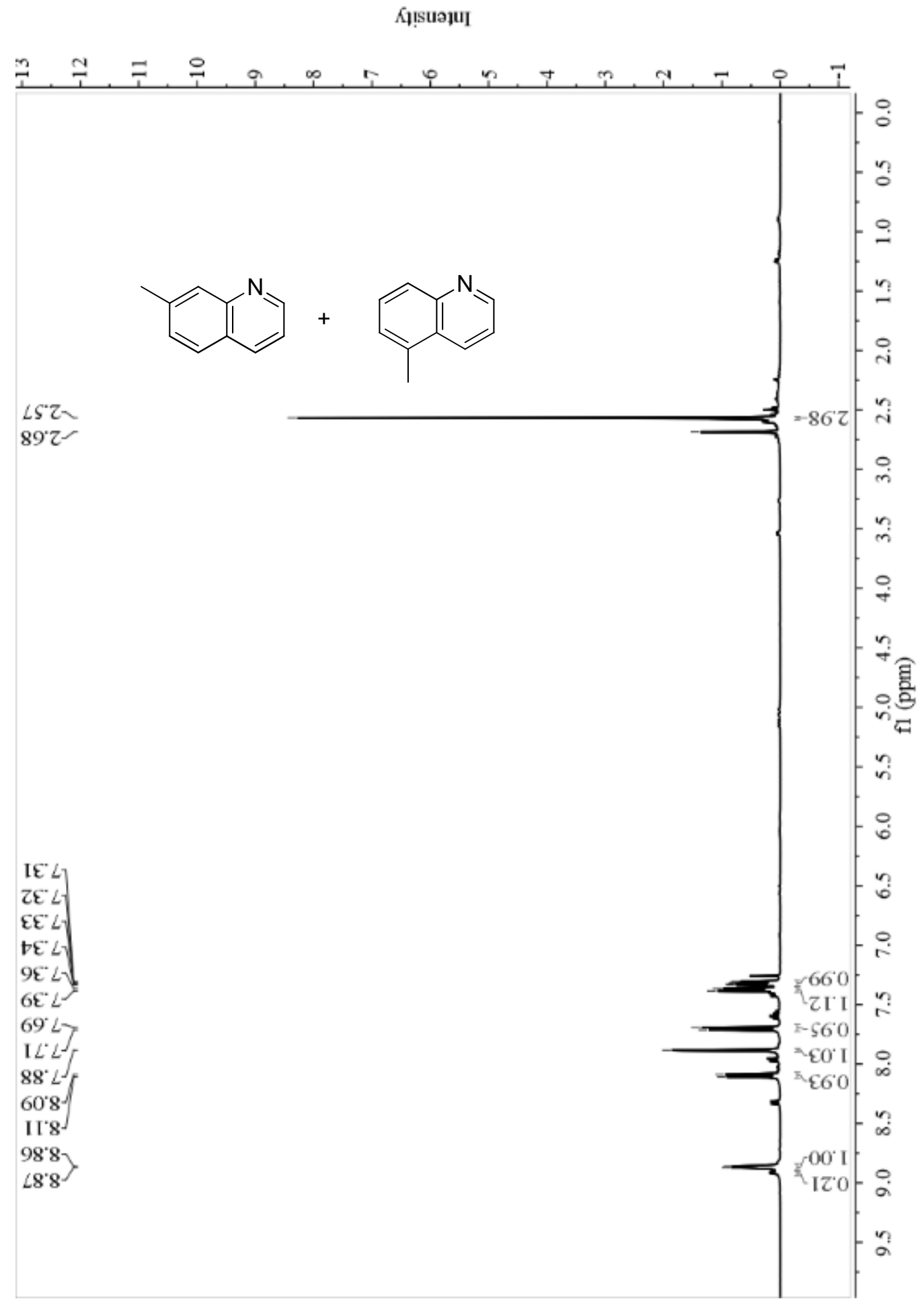

${ }^{1} \mathrm{H}$ NMR of Mixture of Compound 3ea and 3'ea 


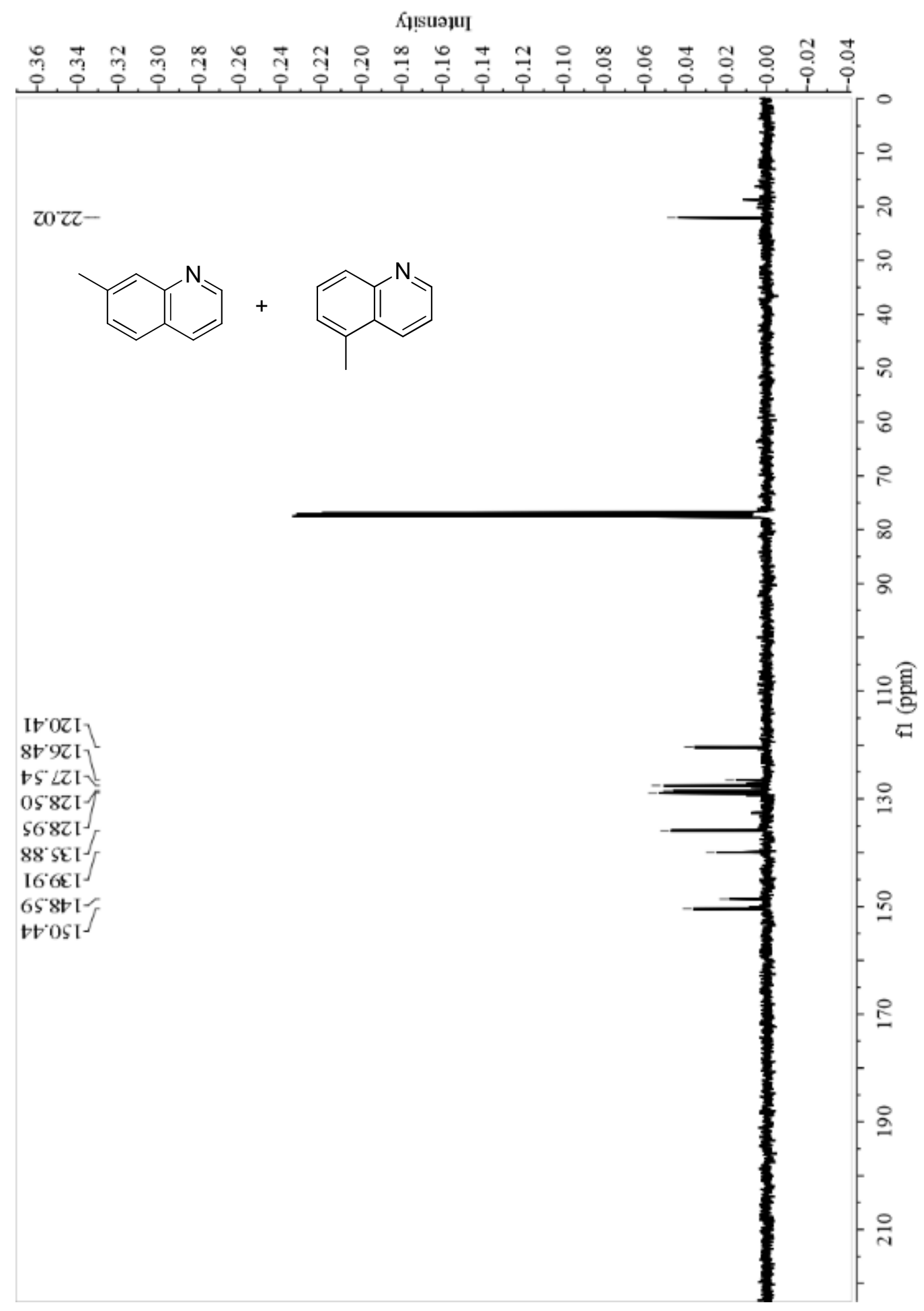

${ }^{13} \mathrm{C}$ NMR of Mixture of Compound 3ea and $\mathbf{3}$ 'ea 


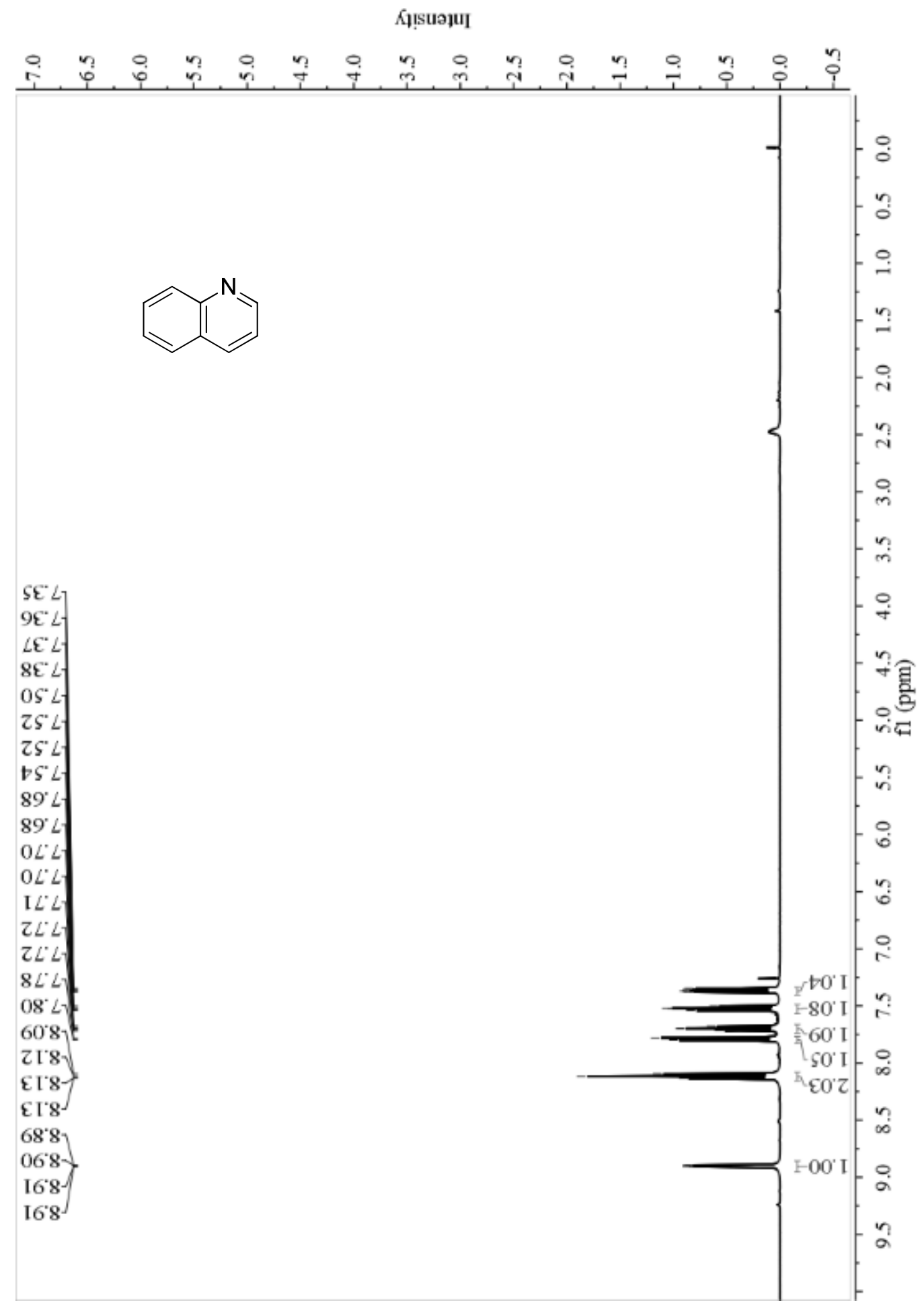

${ }^{1} \mathrm{H}$ NMR of Compound $\mathbf{3 f a}$ 


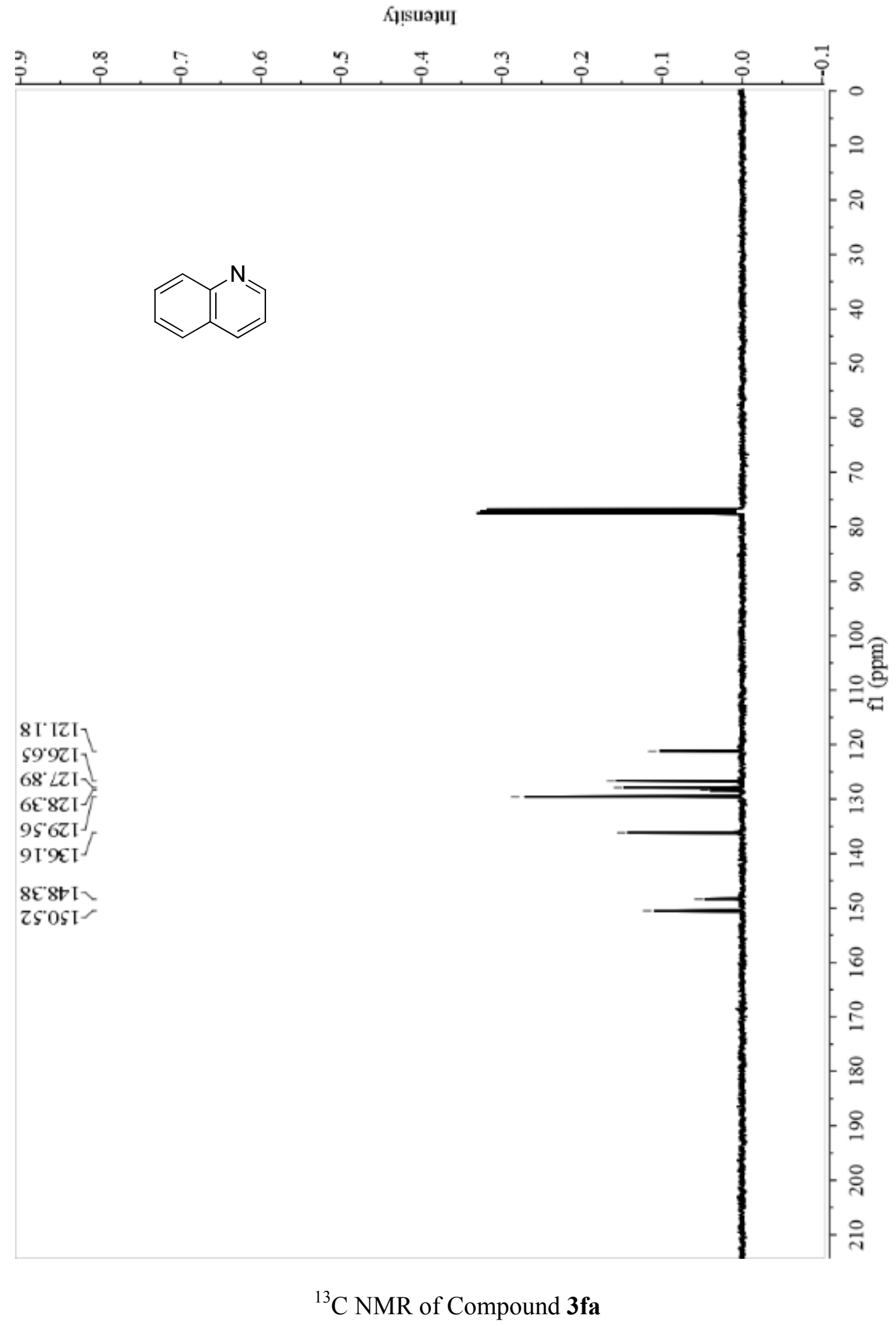




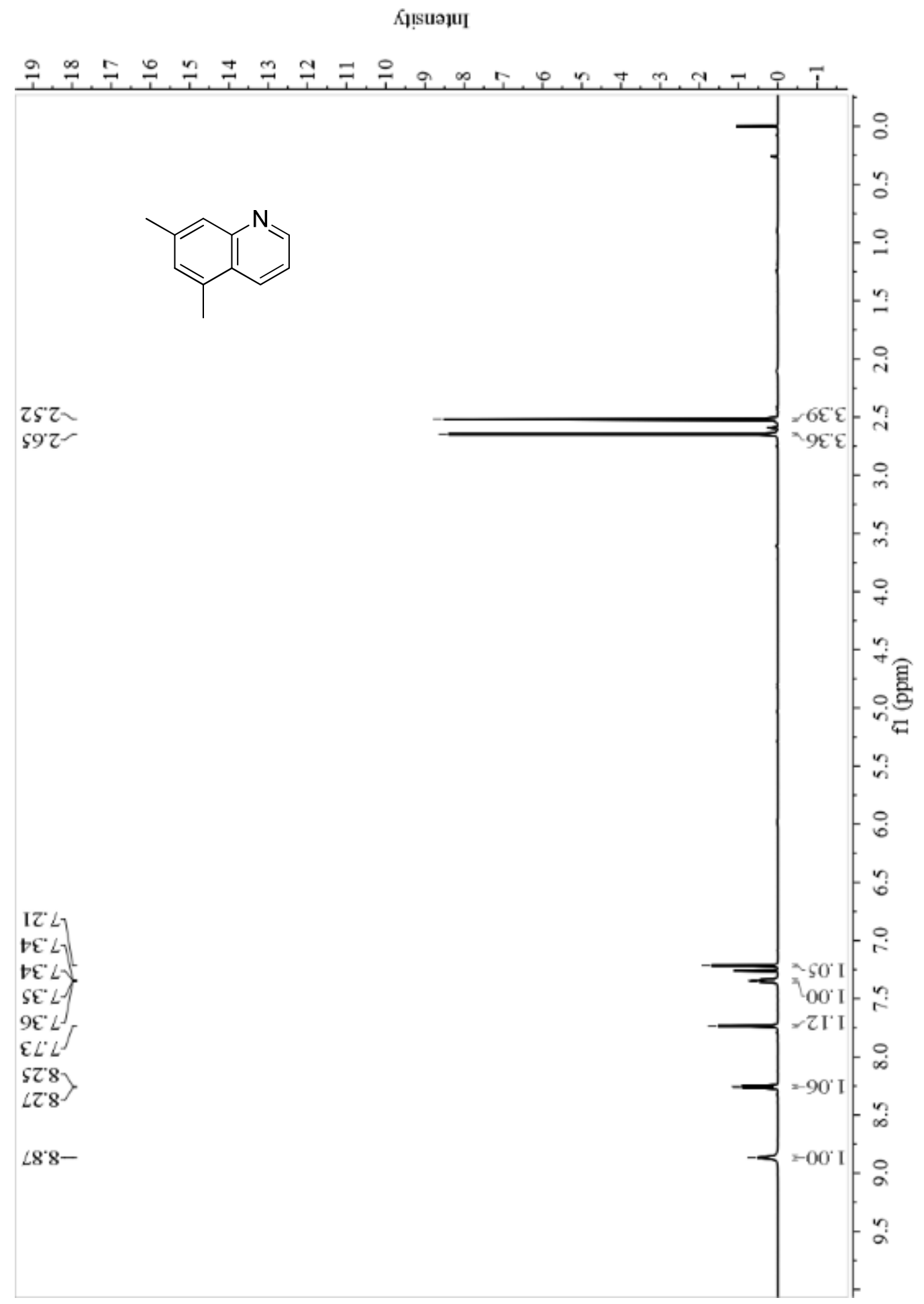

${ }^{1} \mathrm{H}$ NMR of Compound 3ga 


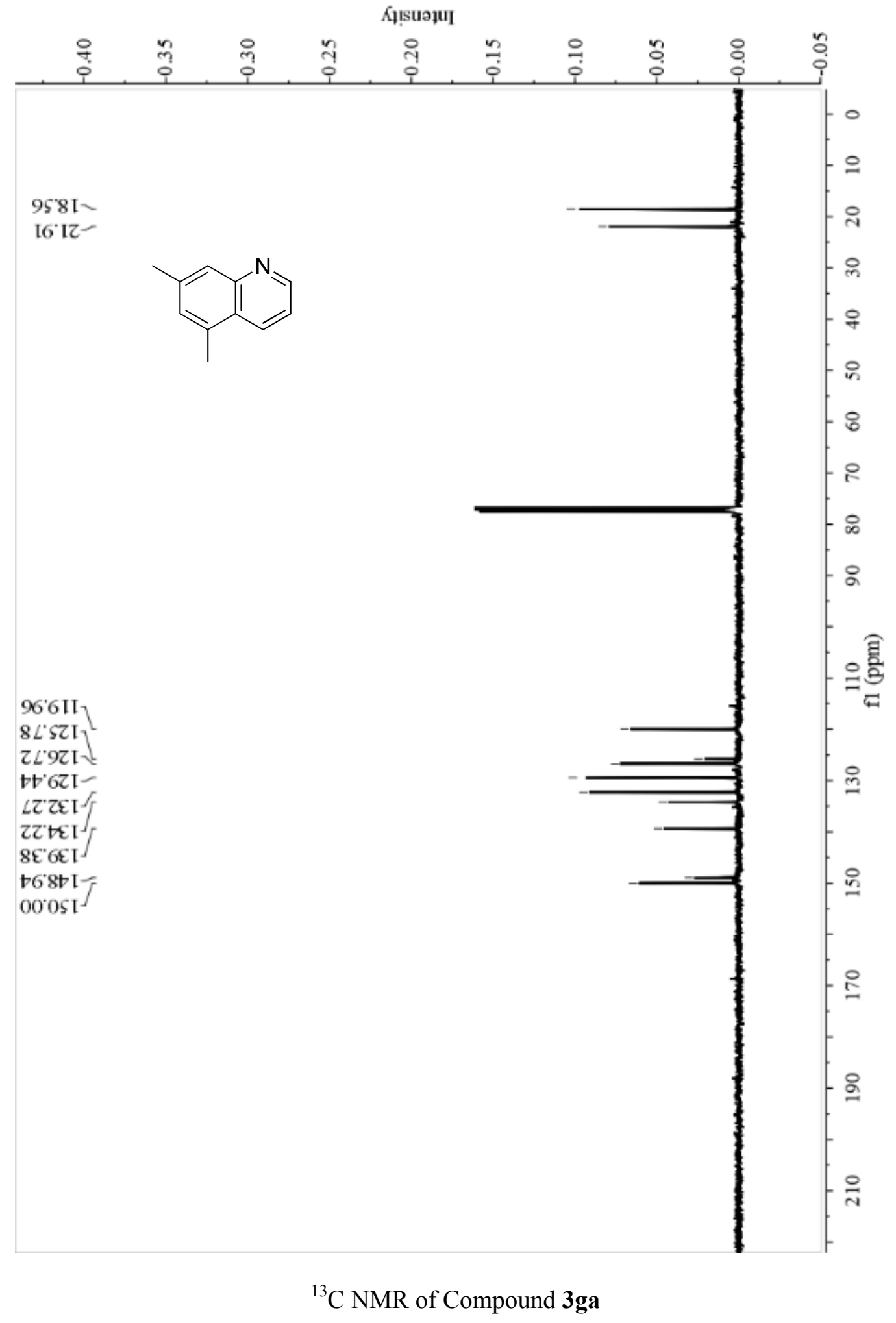




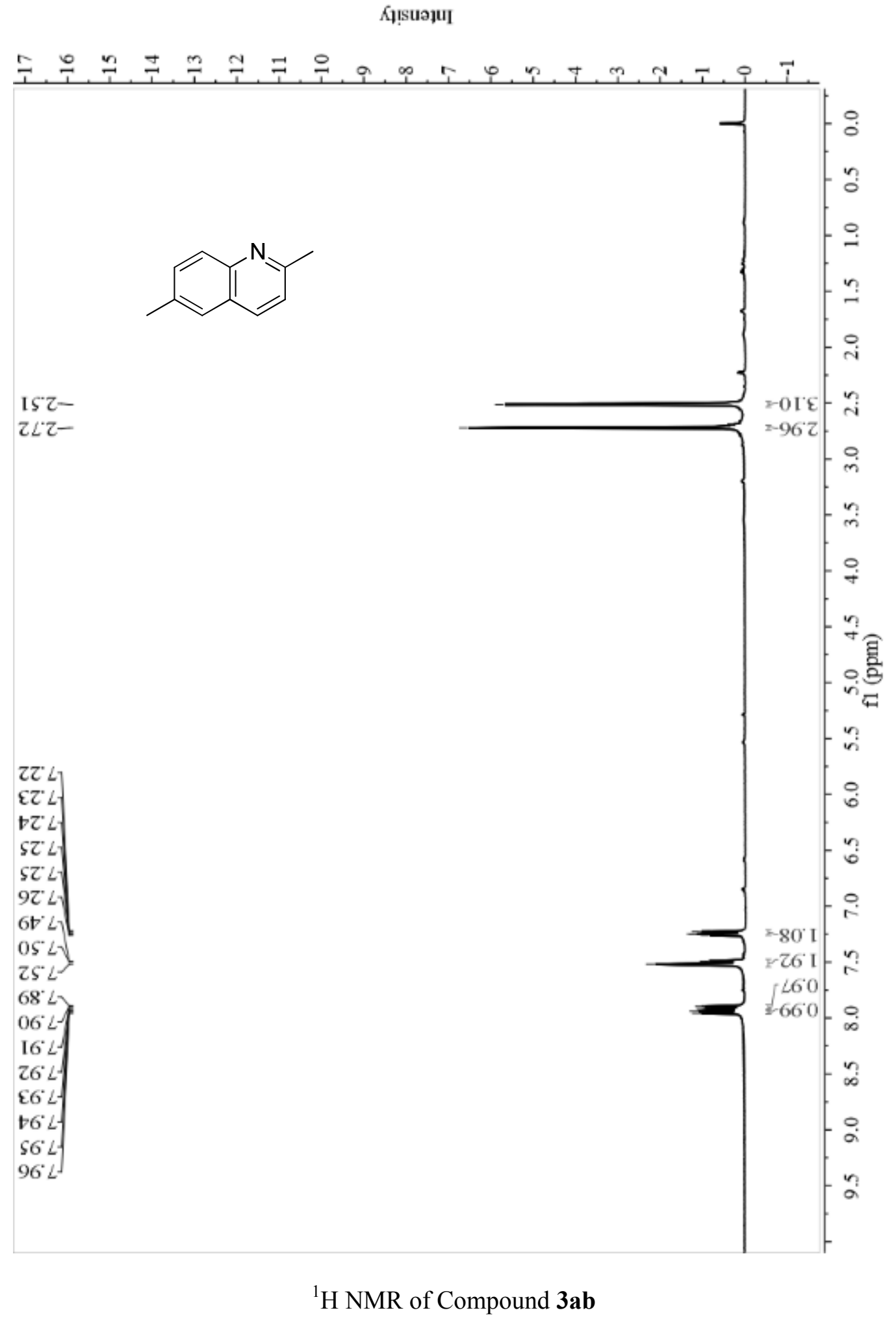


$65^{\circ} \mathrm{I} z-$

$\angle \varepsilon+s z-$

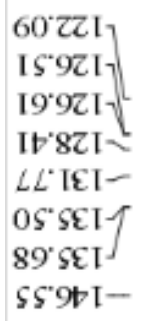

II'8SI-<smiles>Cc1ccc2nc(C)ccc2c1</smiles>

${ }^{13} \mathrm{C}$ NMR of Compound 3ab 


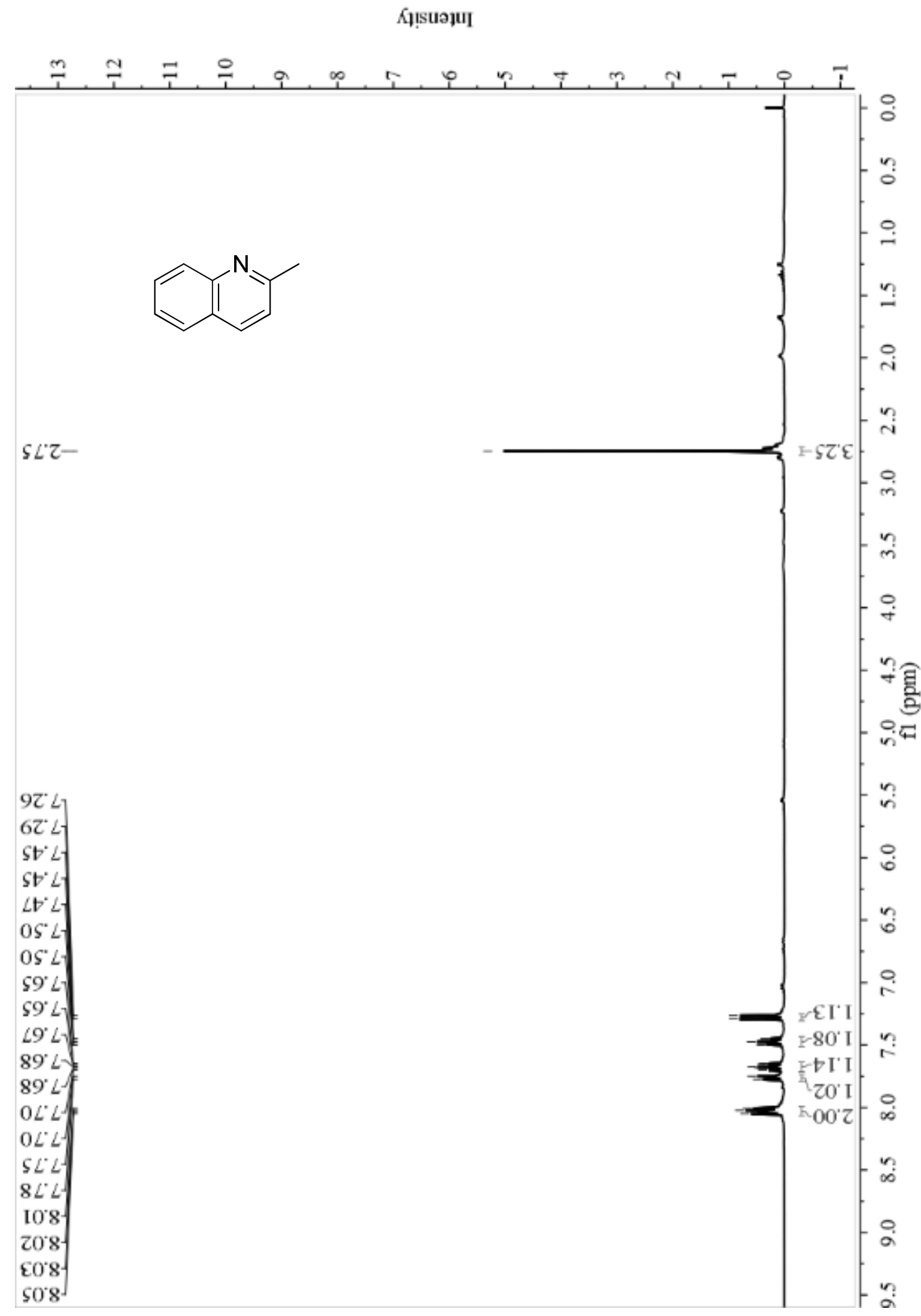

${ }^{1}$ H NMR of Compound $\mathbf{3 f b}$ 


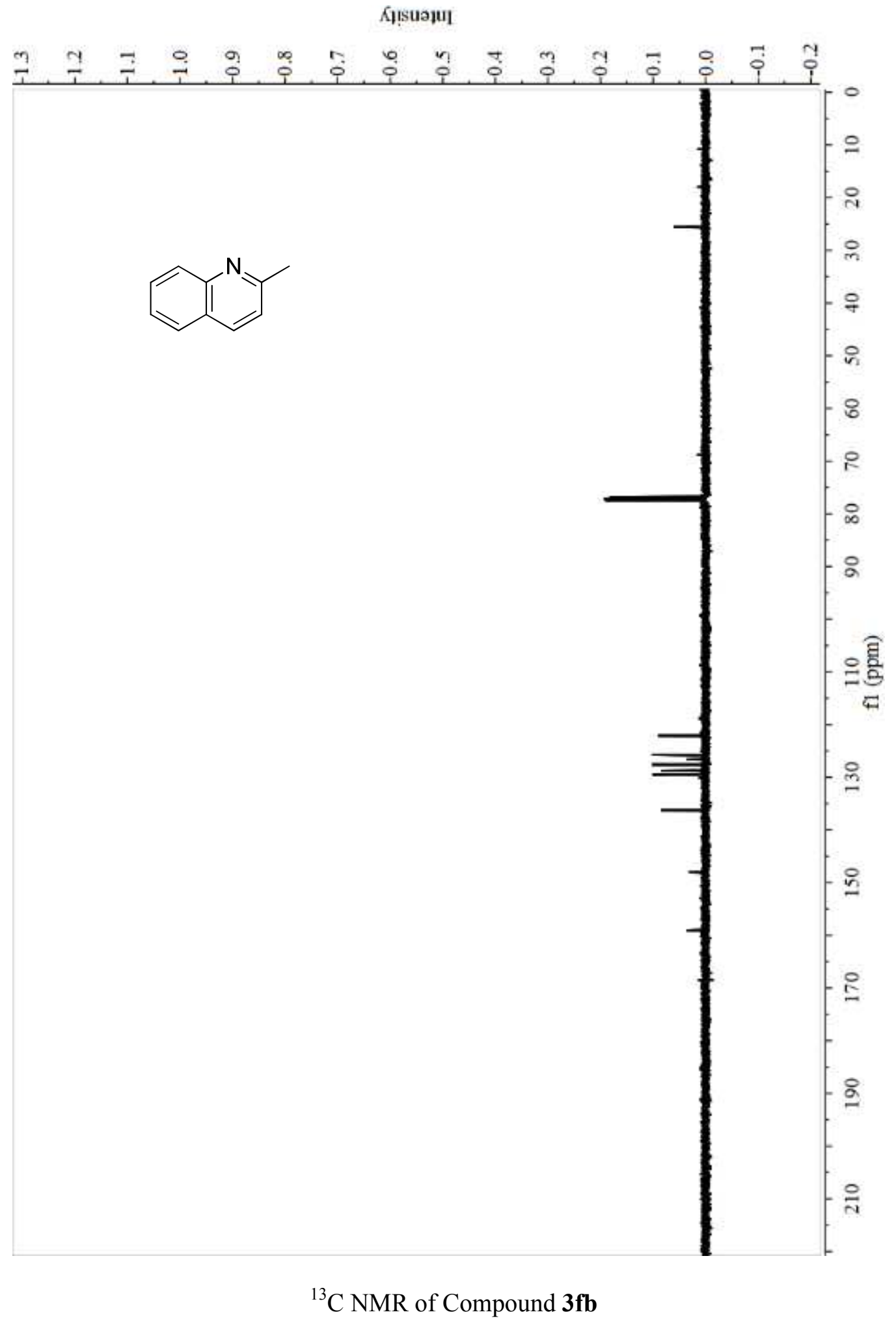




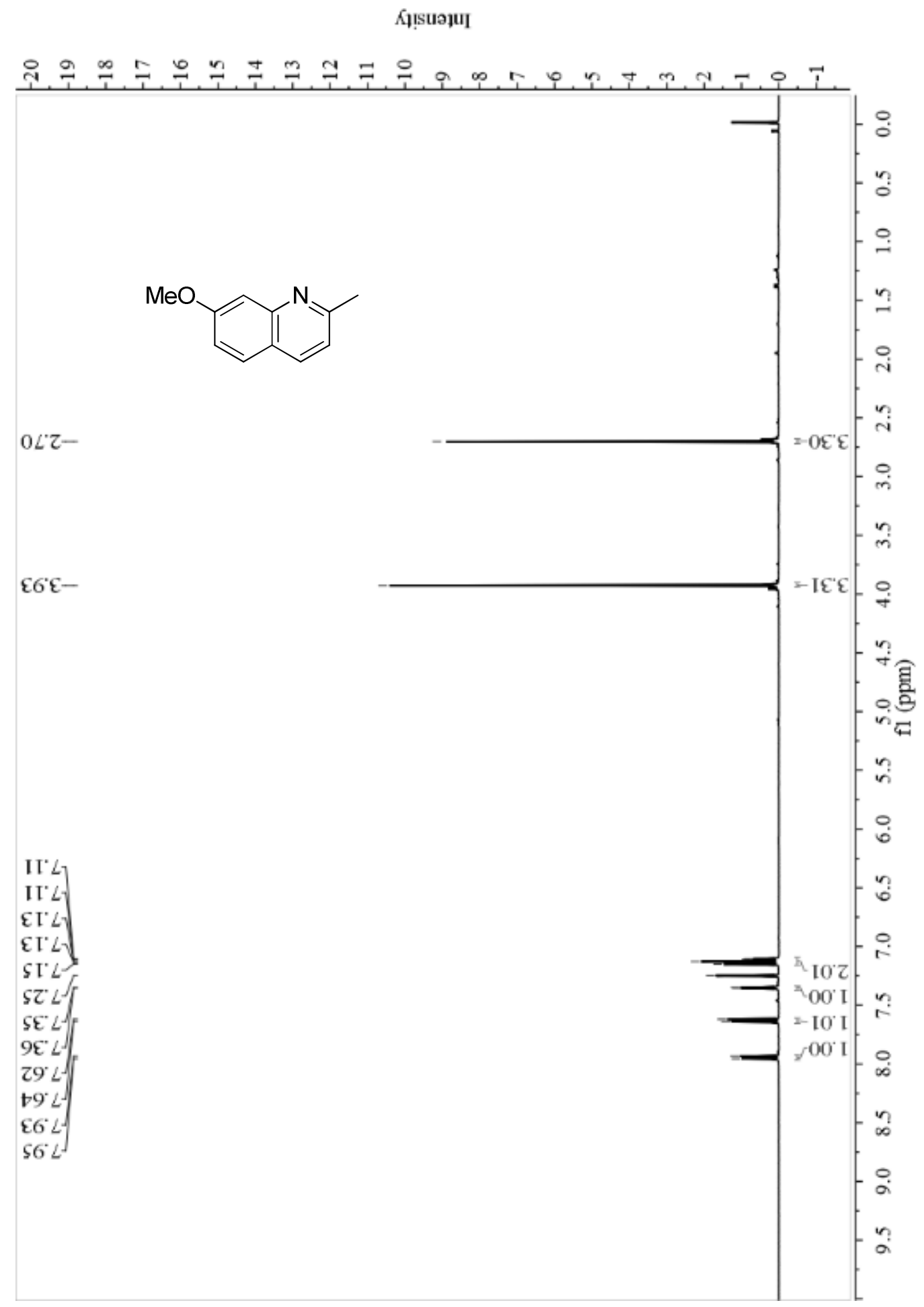

${ }^{1} \mathrm{H}$ NMR of Compound $\mathbf{3 h b}$ 


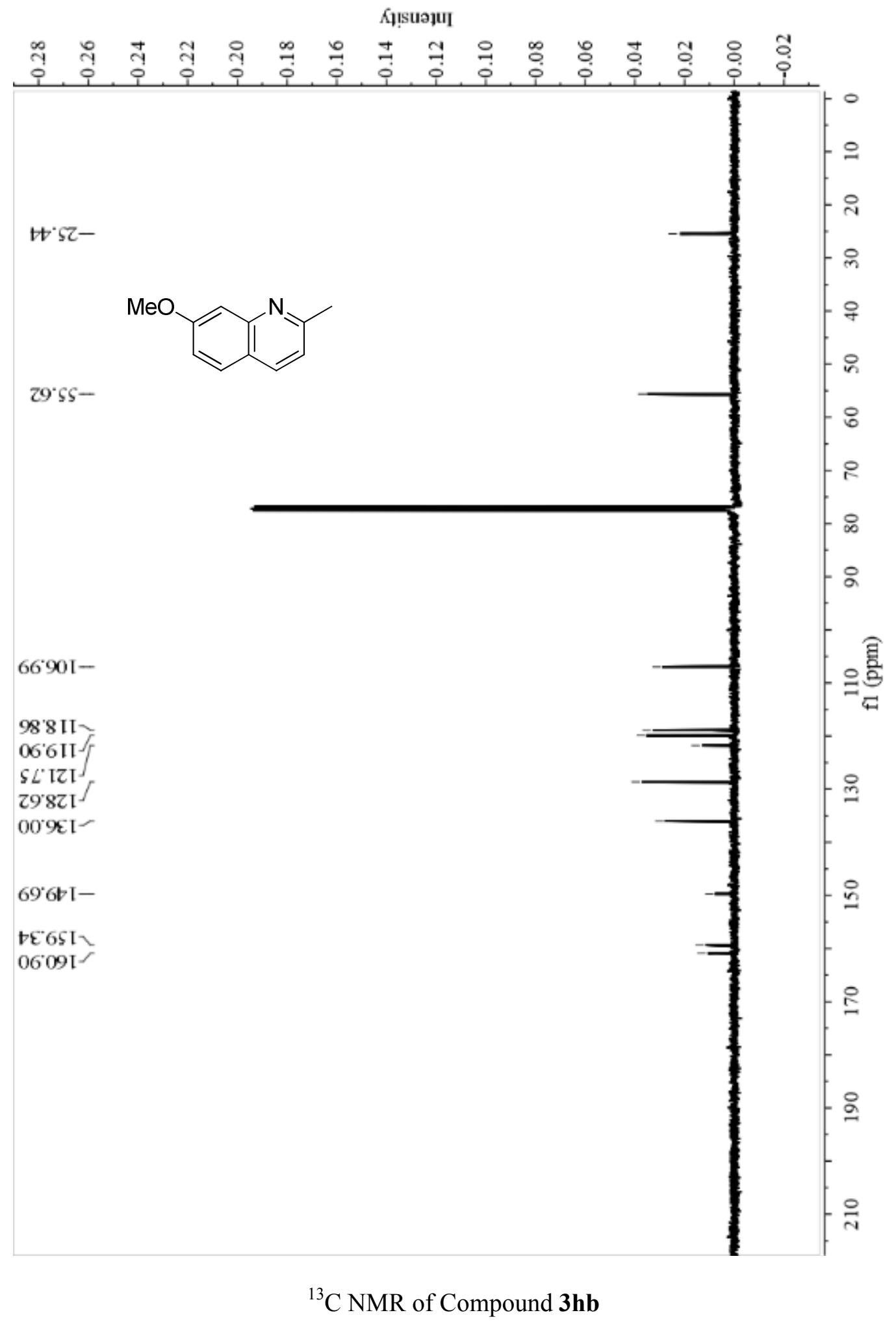




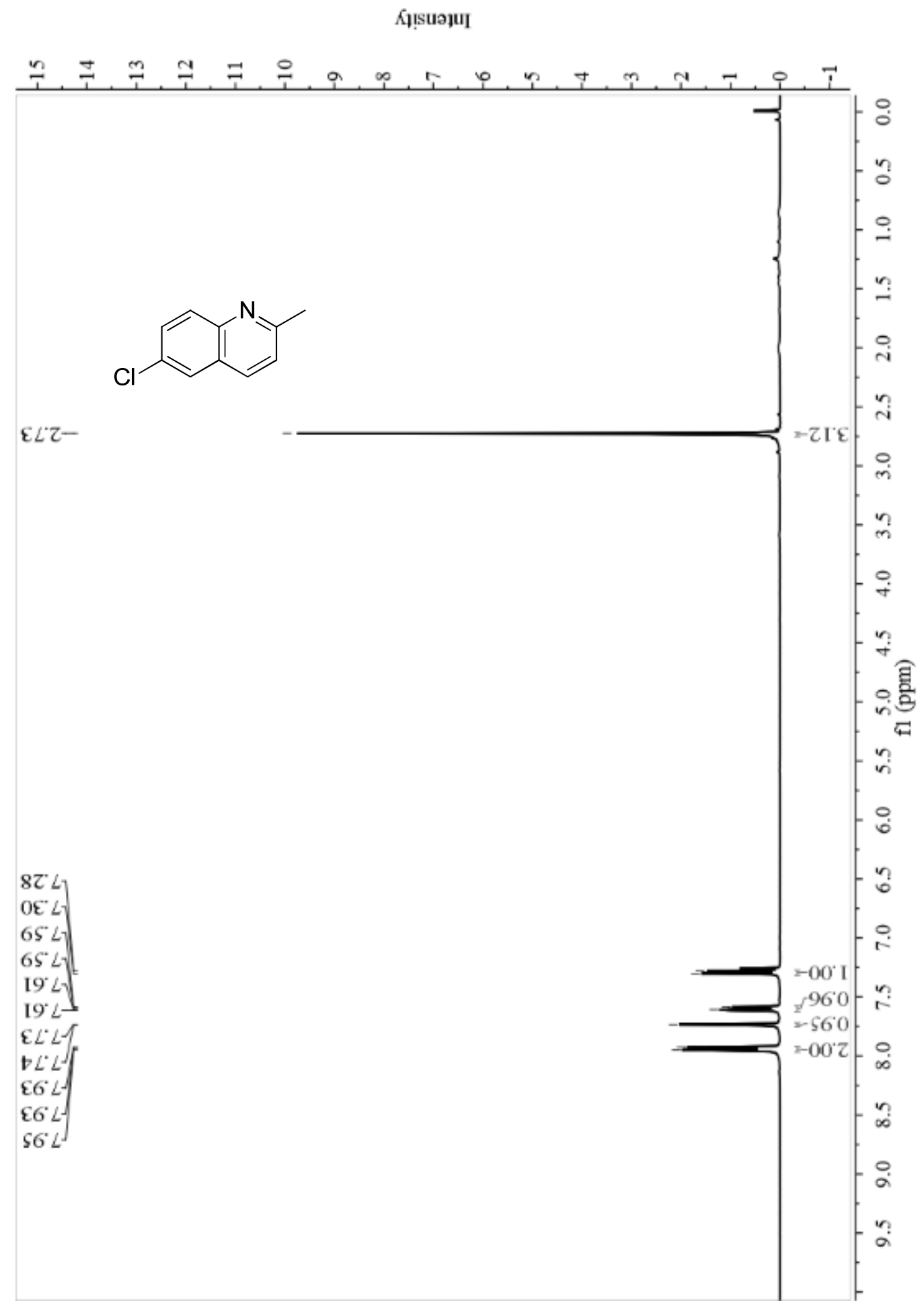

${ }^{1} \mathrm{H}$ NMR of Compound 3ib 


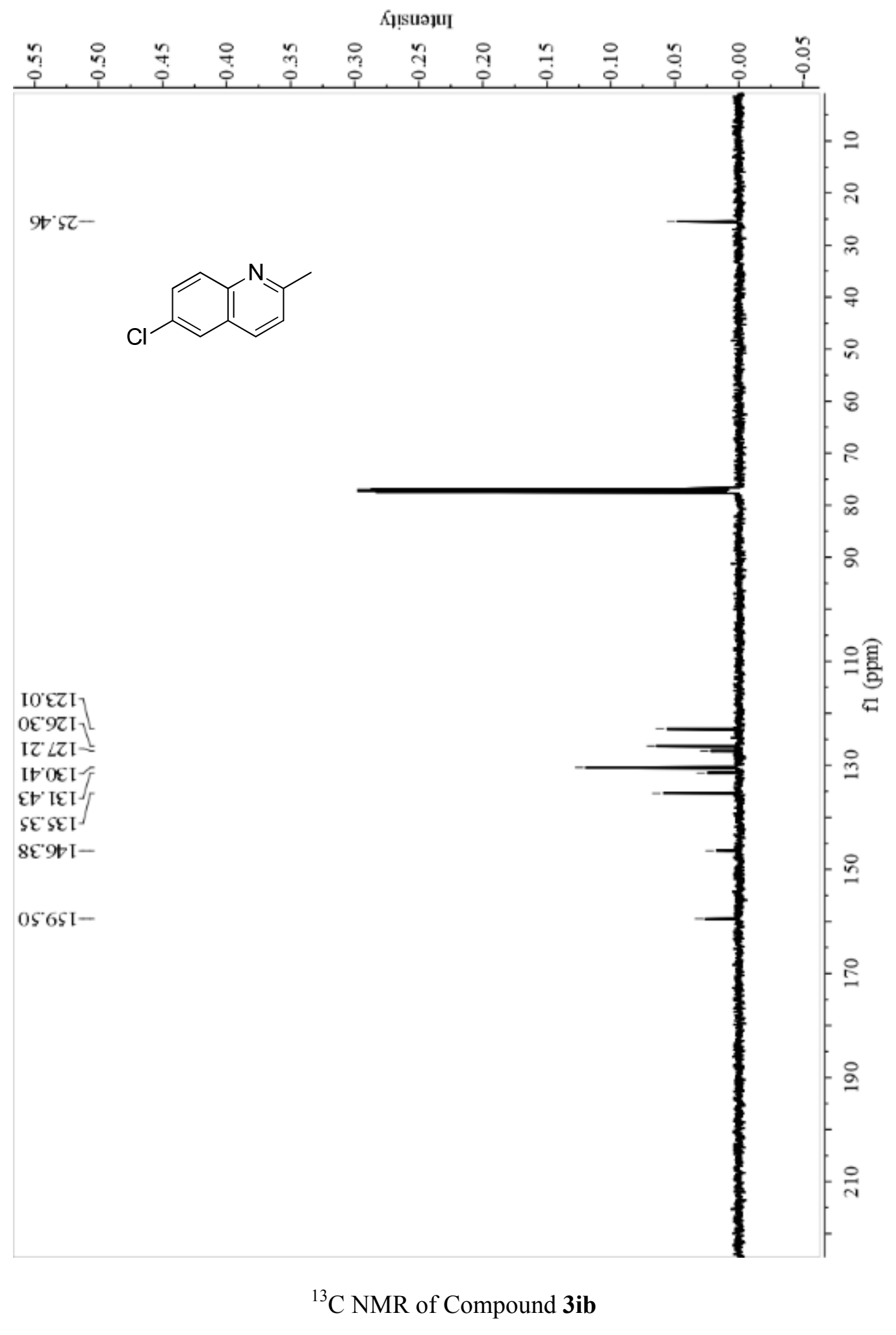




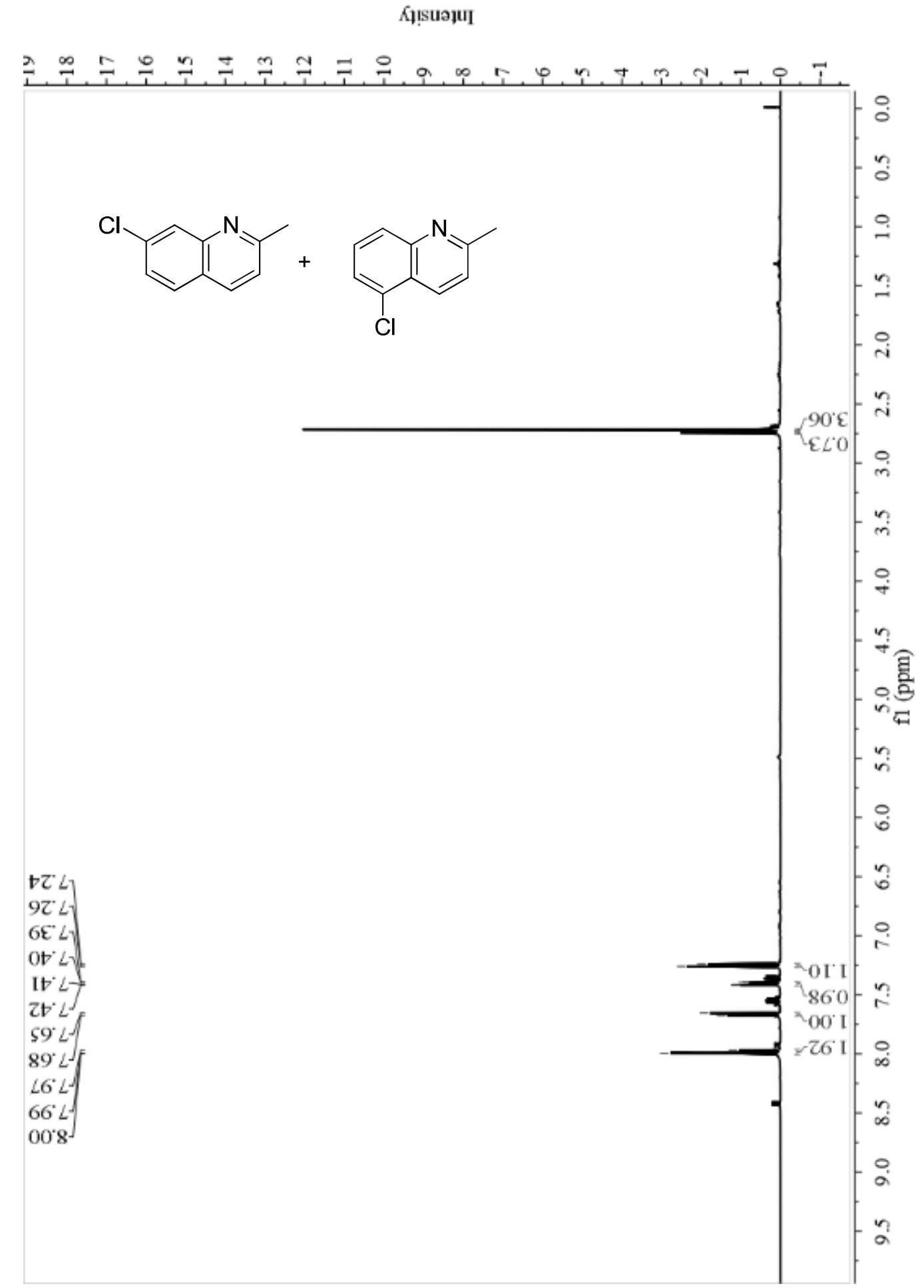

${ }^{1} \mathrm{H}$ NMR of Mixture of Compound $\mathbf{3 j b}$ and $\mathbf{3} \mathbf{j} \mathbf{j b}$ 


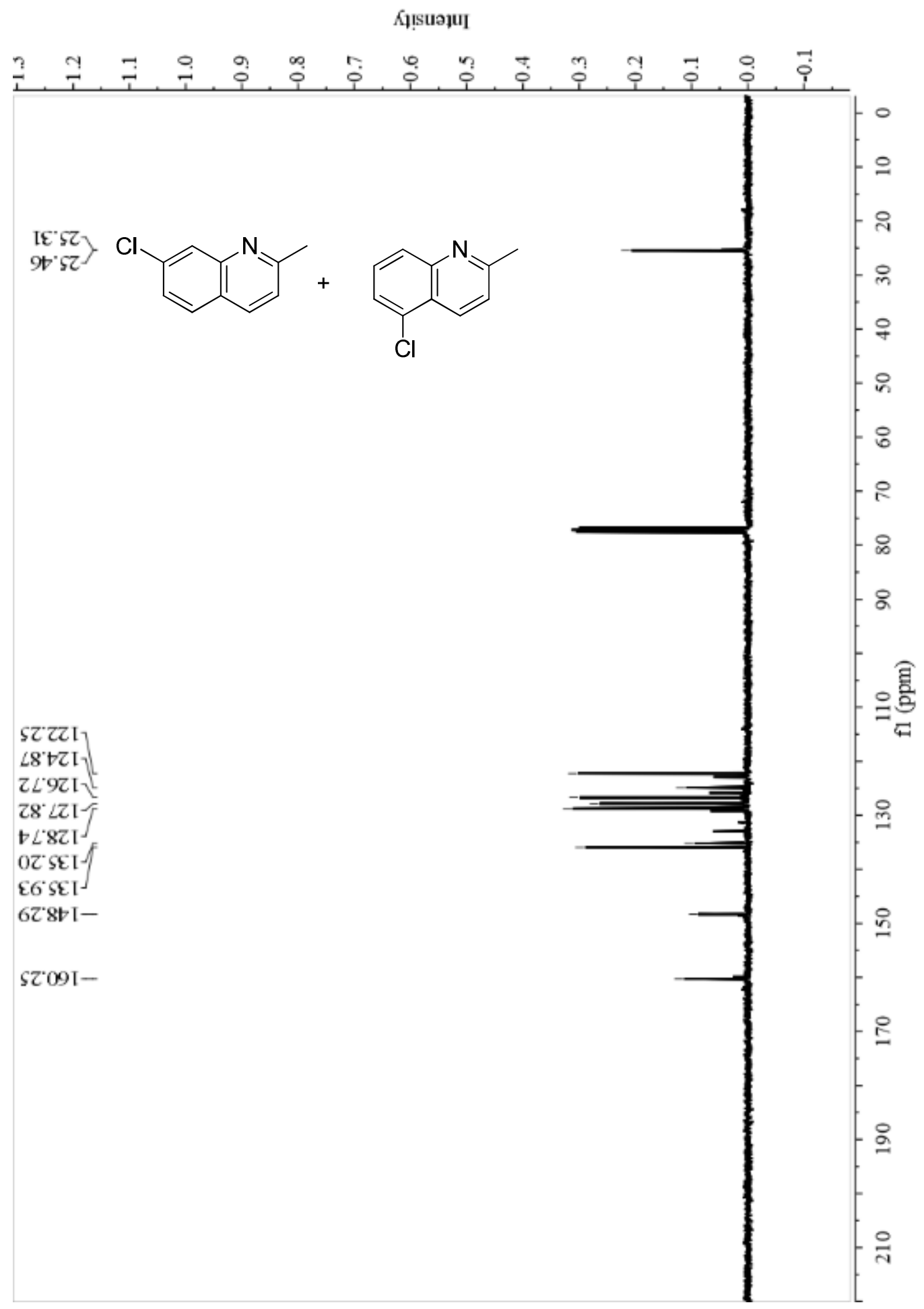

${ }^{13} \mathrm{C}$ NMR of Mixture of Compound $\mathbf{3 j b}$ and $\mathbf{3}$ 'jb 


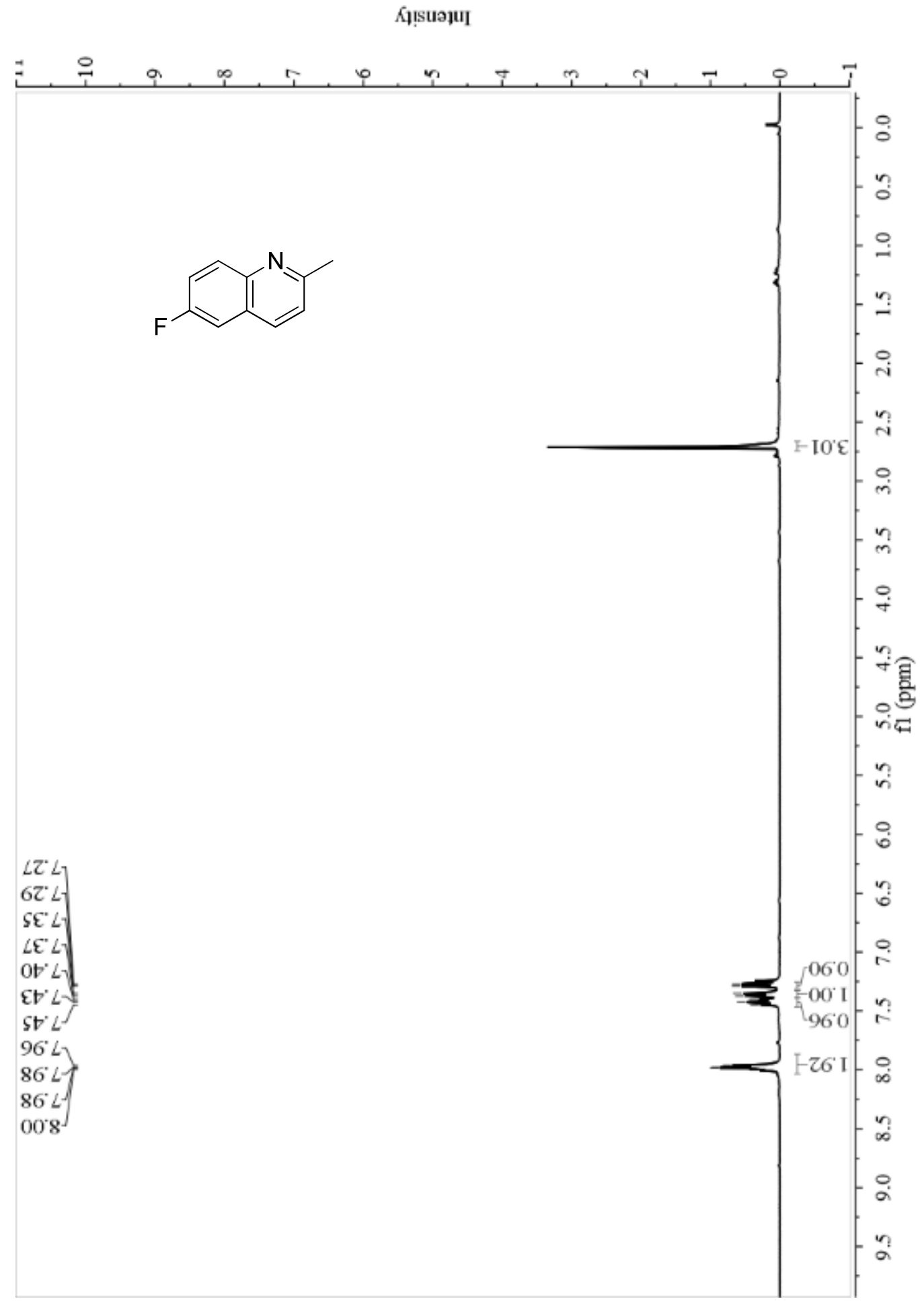

${ }^{1} \mathrm{H}$ NMR of Compound $\mathbf{3 k b}$ 


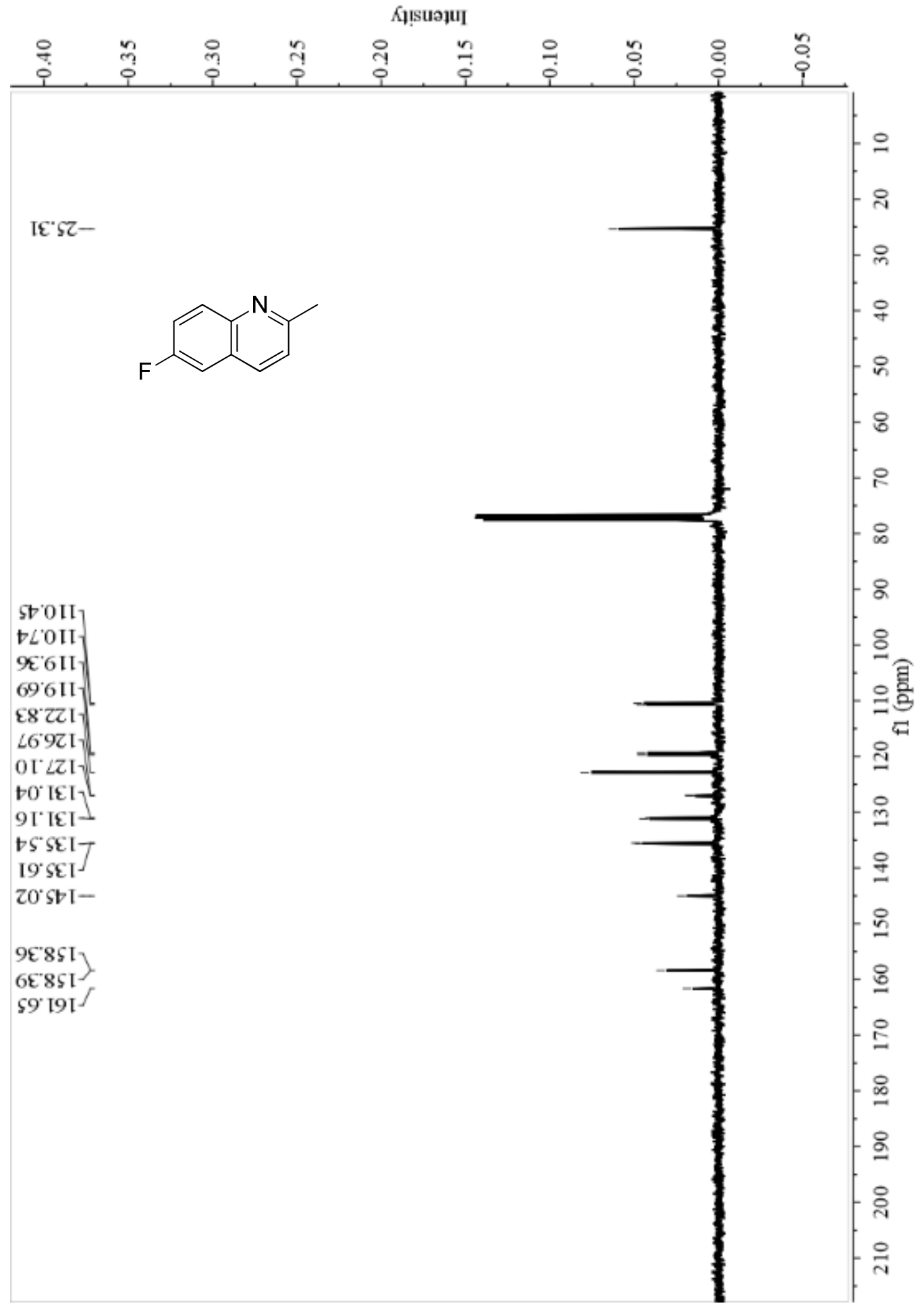

${ }^{13} \mathrm{C}$ NMR of Compound $\mathbf{3 k b}$ 


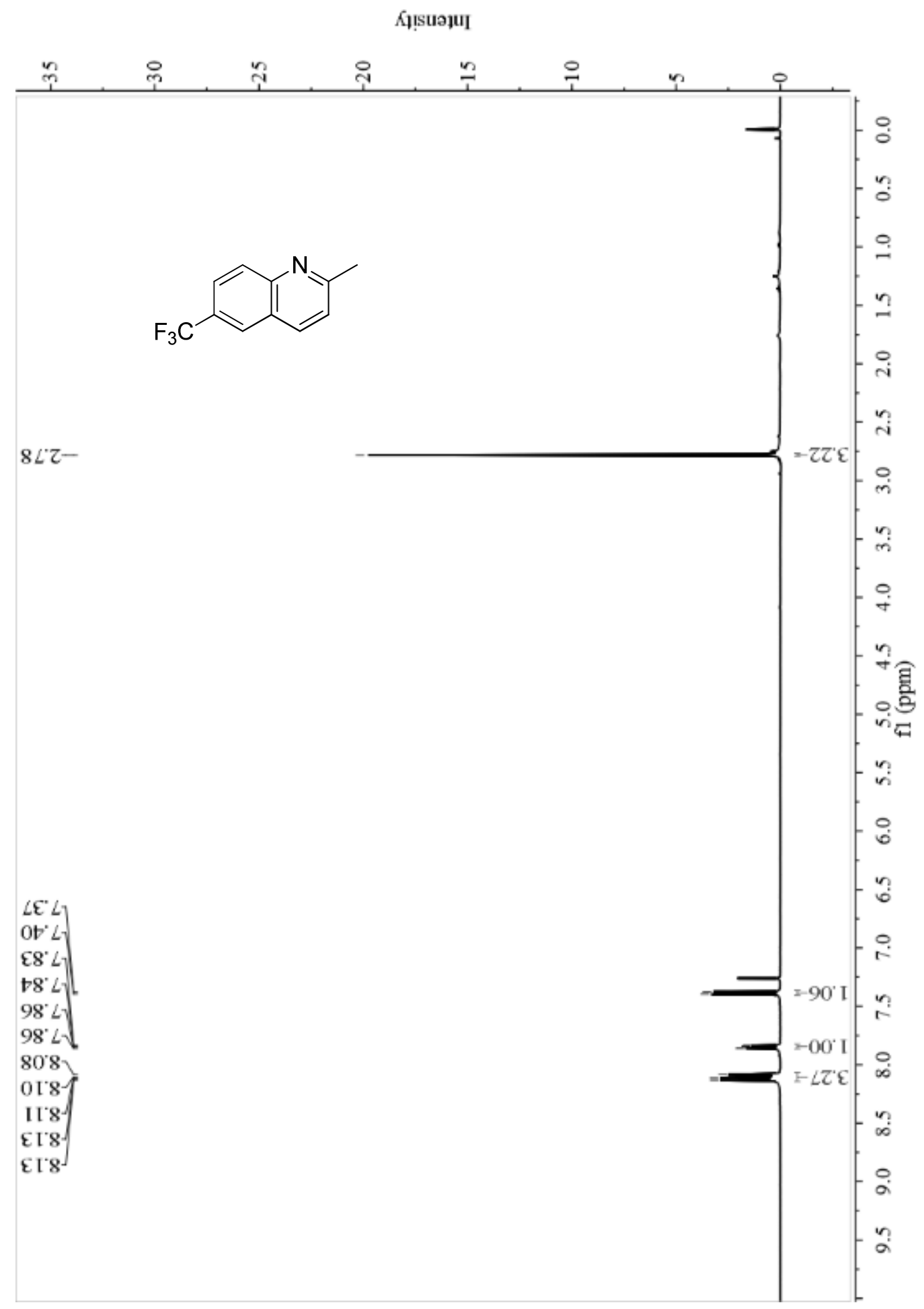

${ }^{1} \mathrm{H}$ NMR of Compound 3lb 


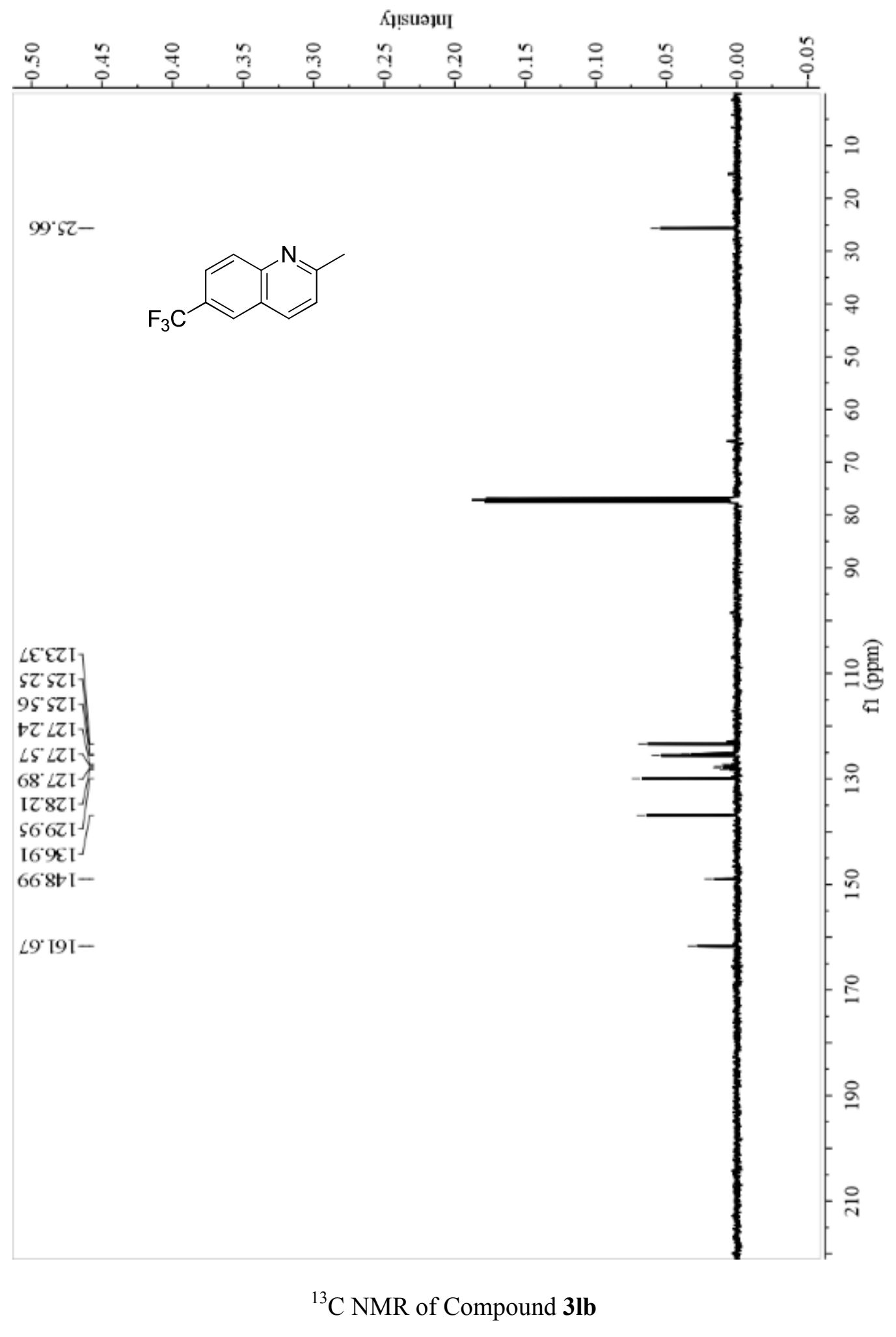




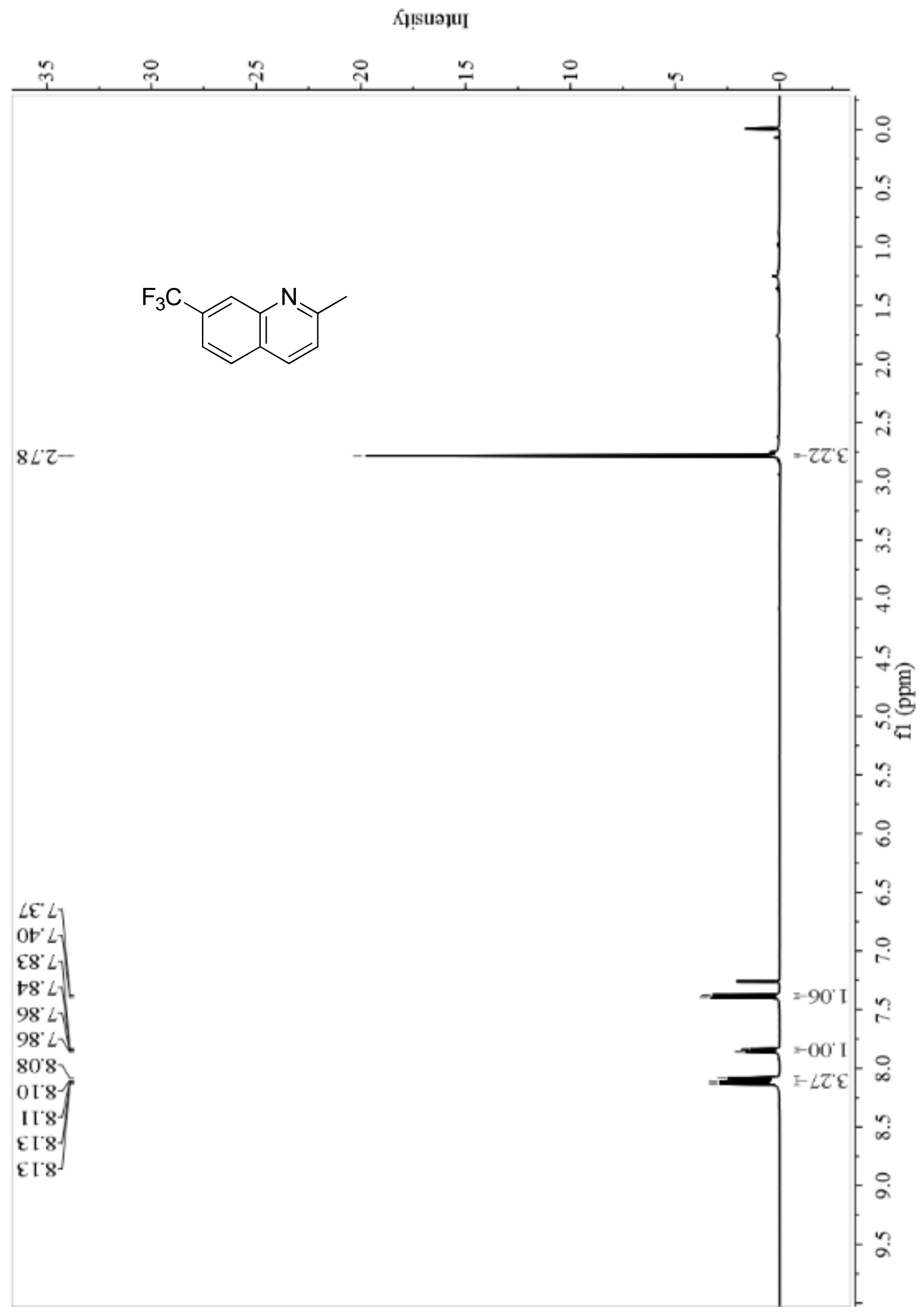

${ }^{1} \mathrm{H}$ NMR of Compound $\mathbf{3} \mathbf{m b}$ 


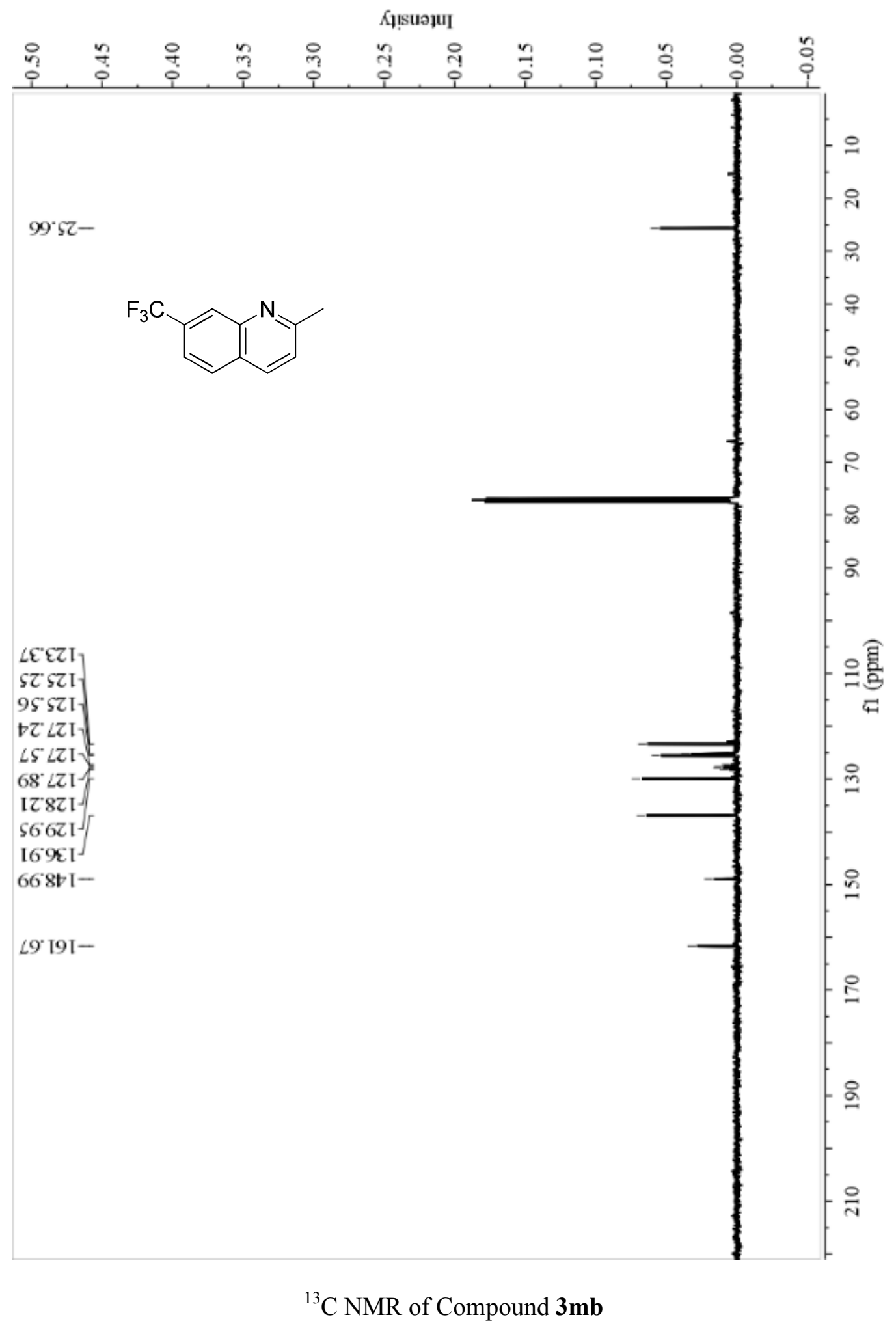




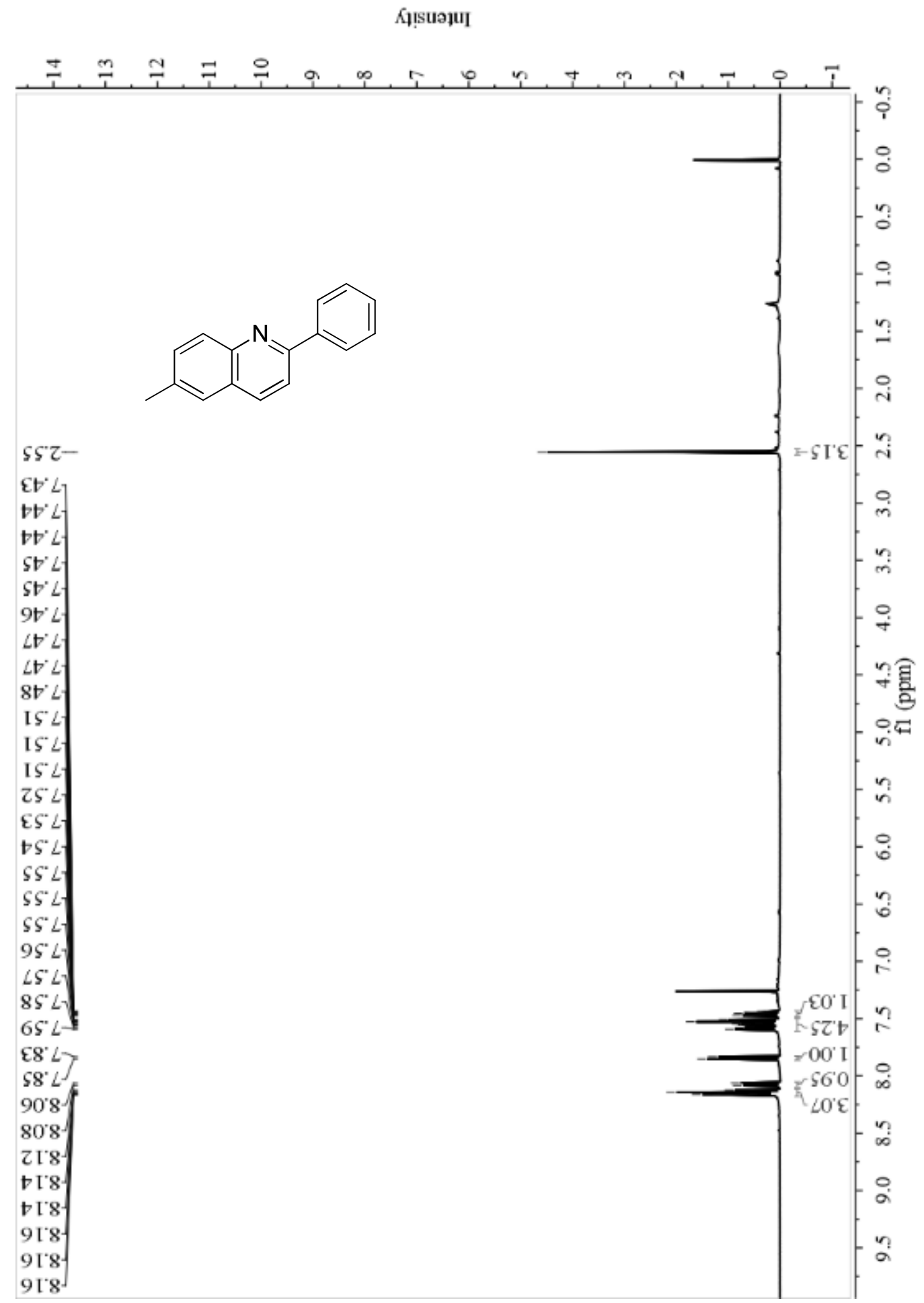

${ }^{1} \mathrm{H}$ NMR of Compound 3ac 


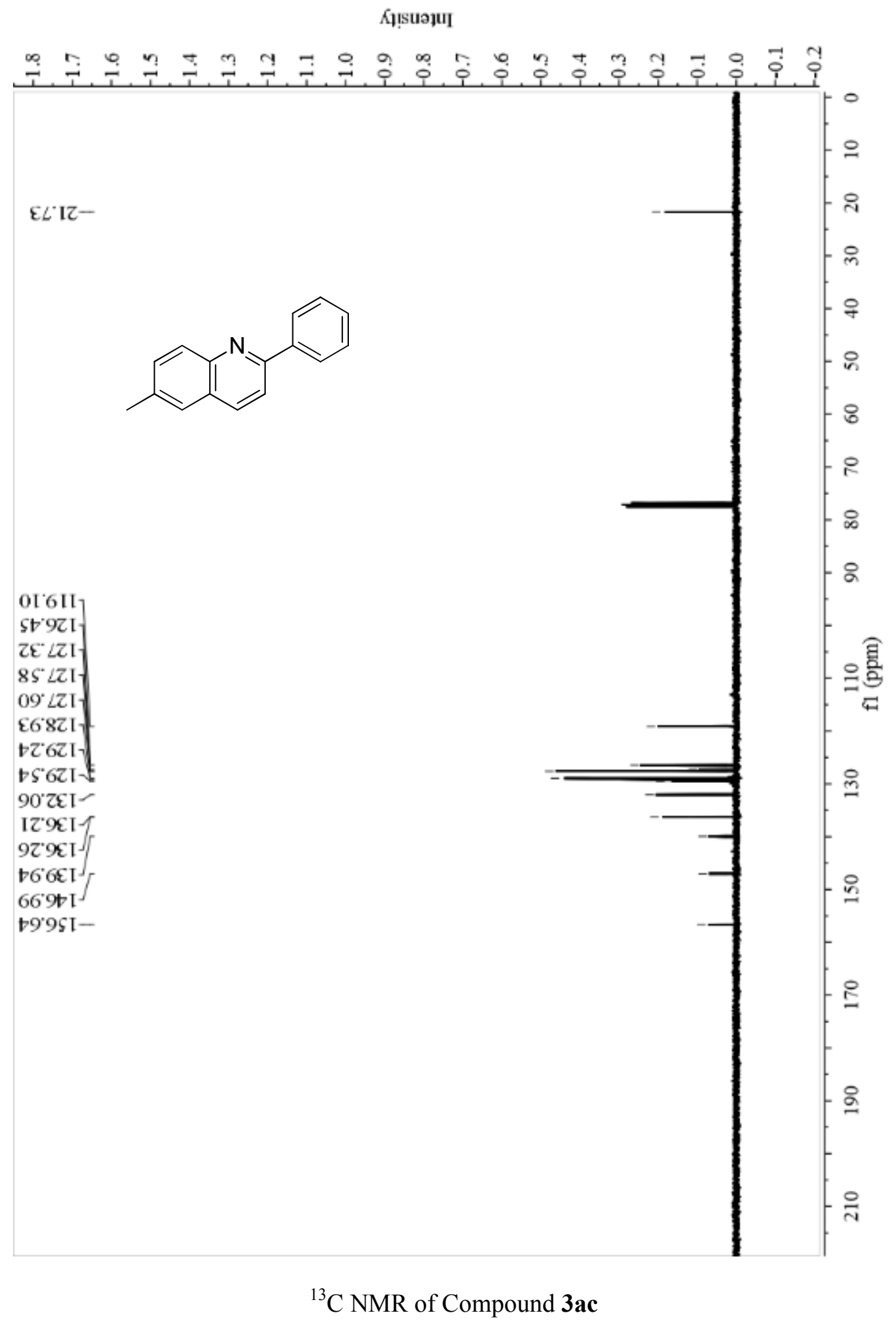




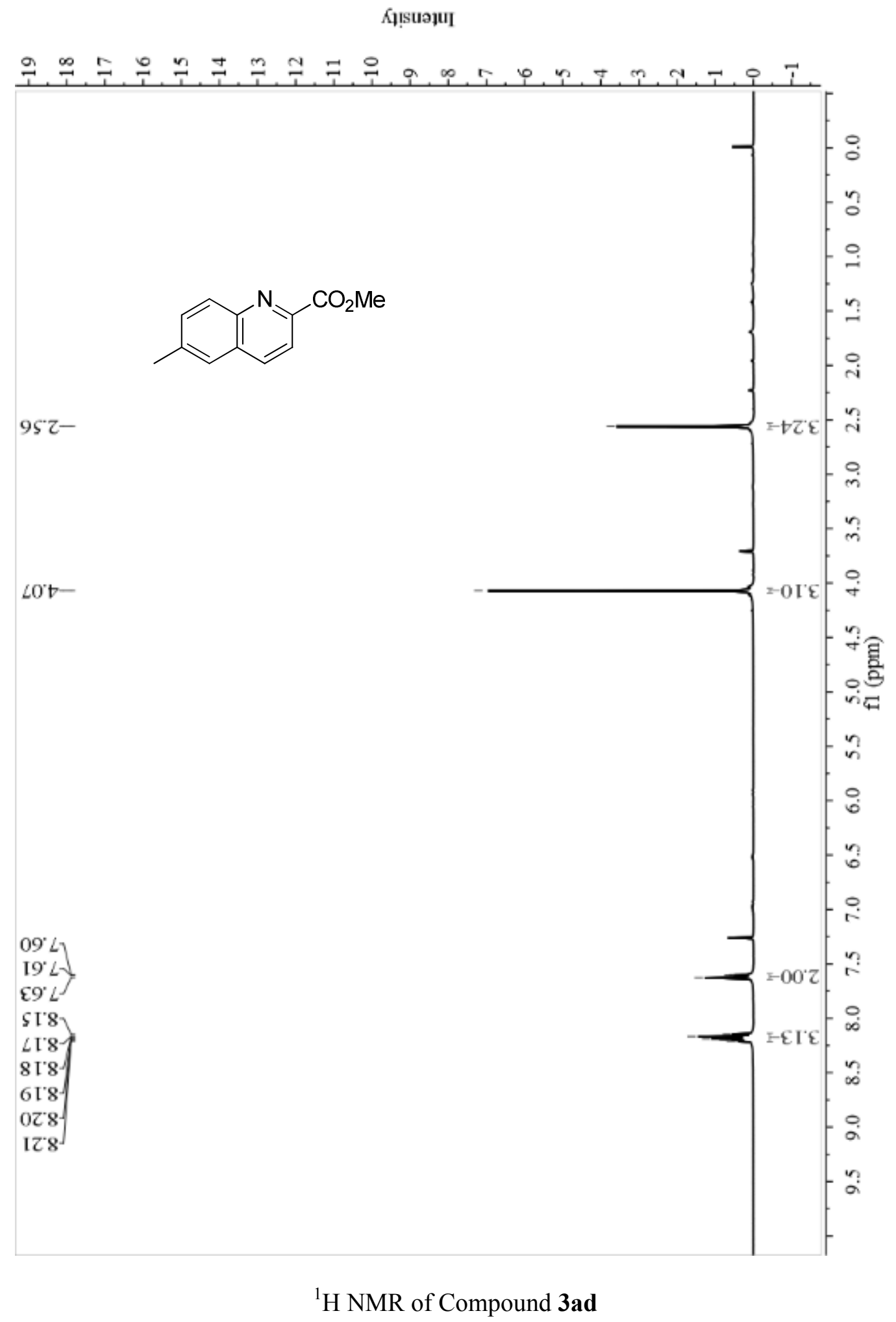




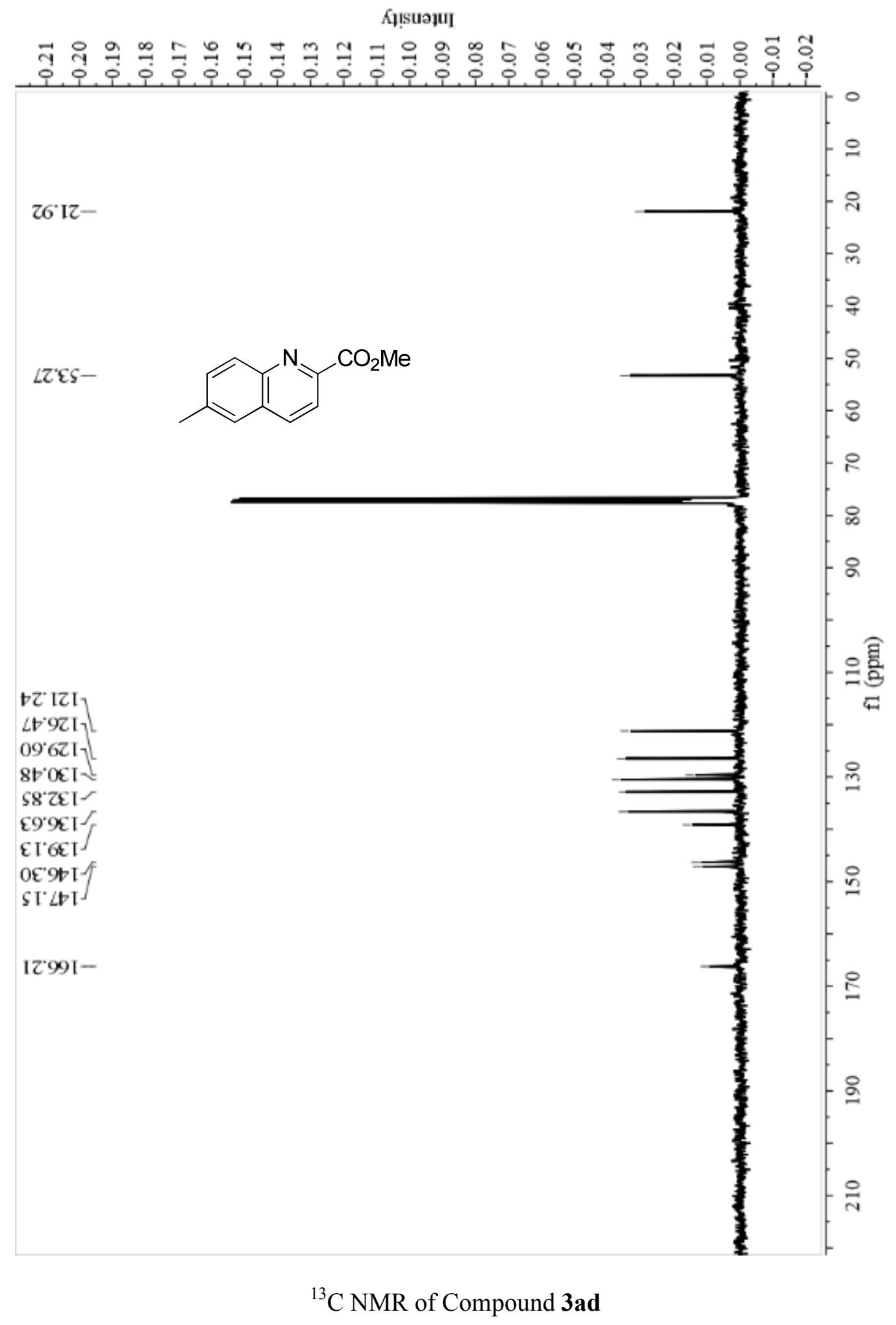




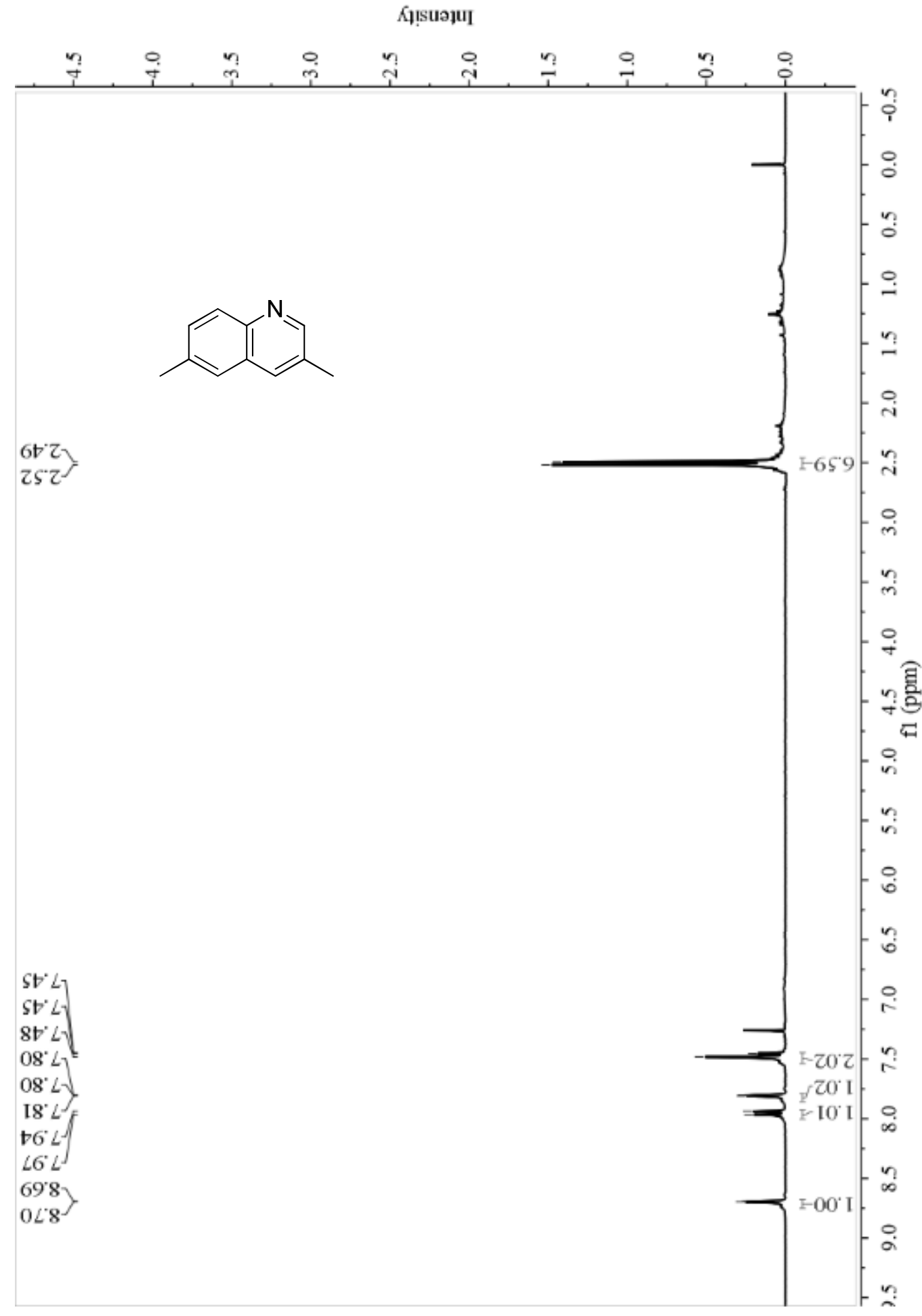

${ }^{1} \mathrm{H}$ NMR of Compound 3ae 


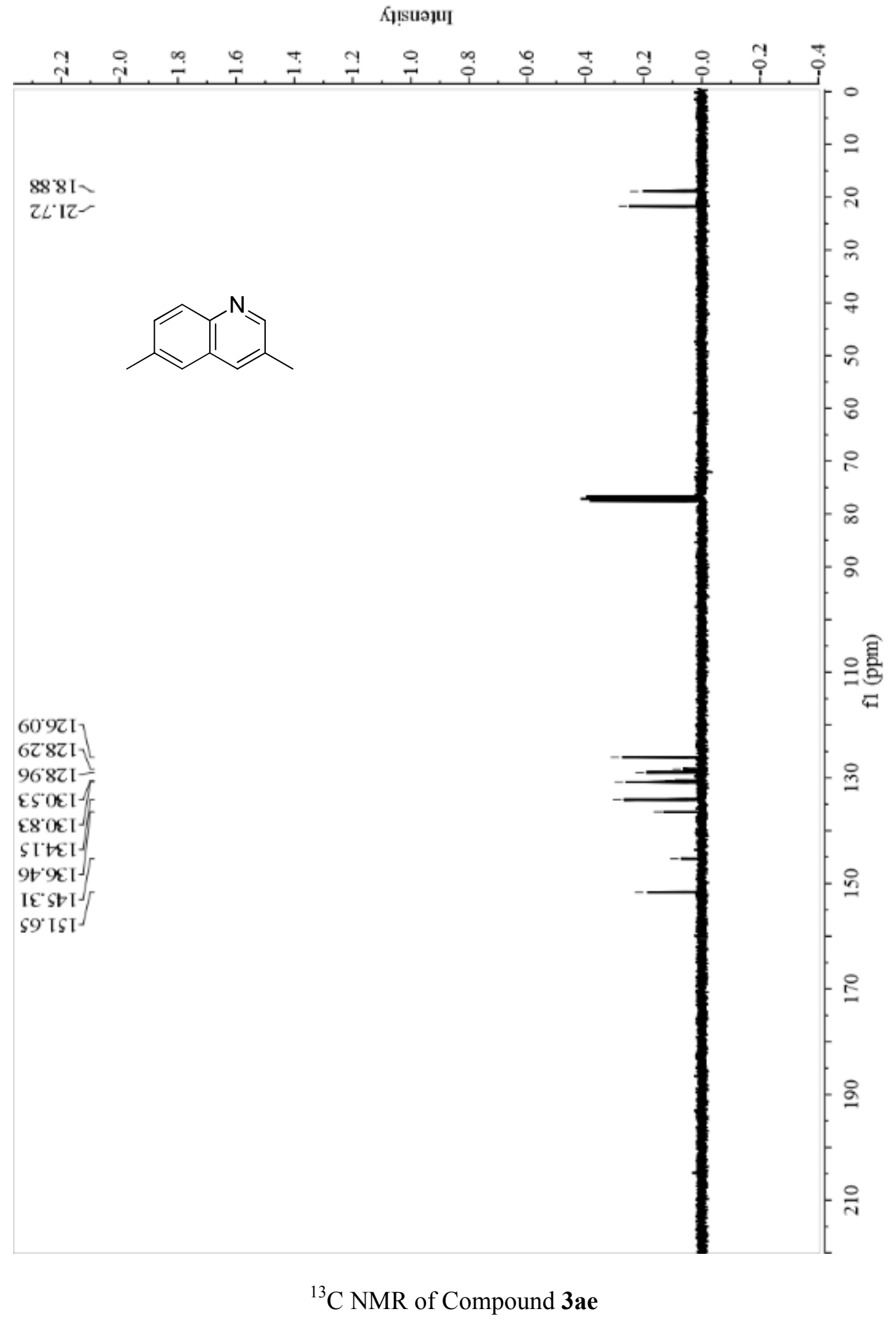




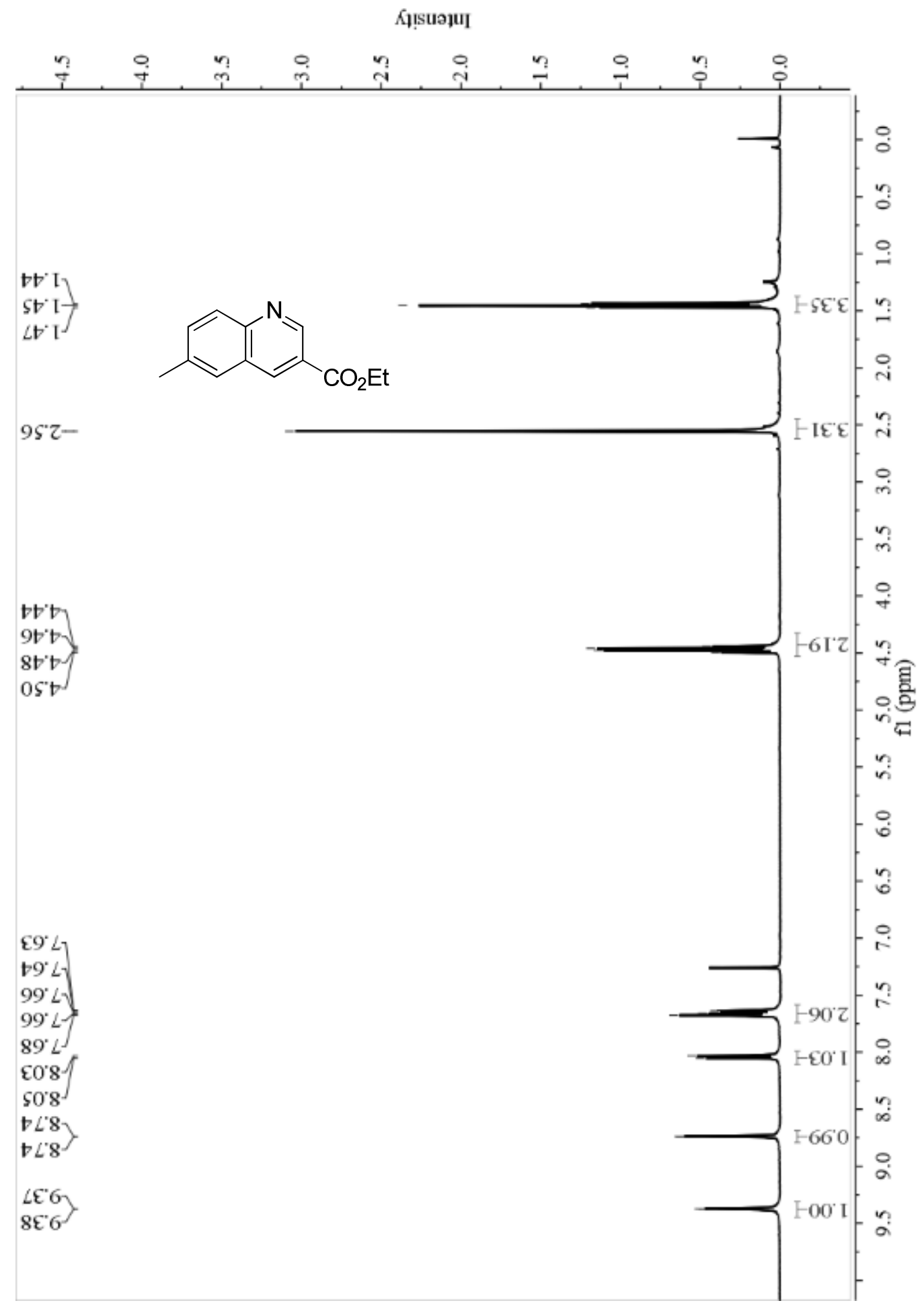

${ }^{1} \mathrm{H}$ NMR of Compound 3af 


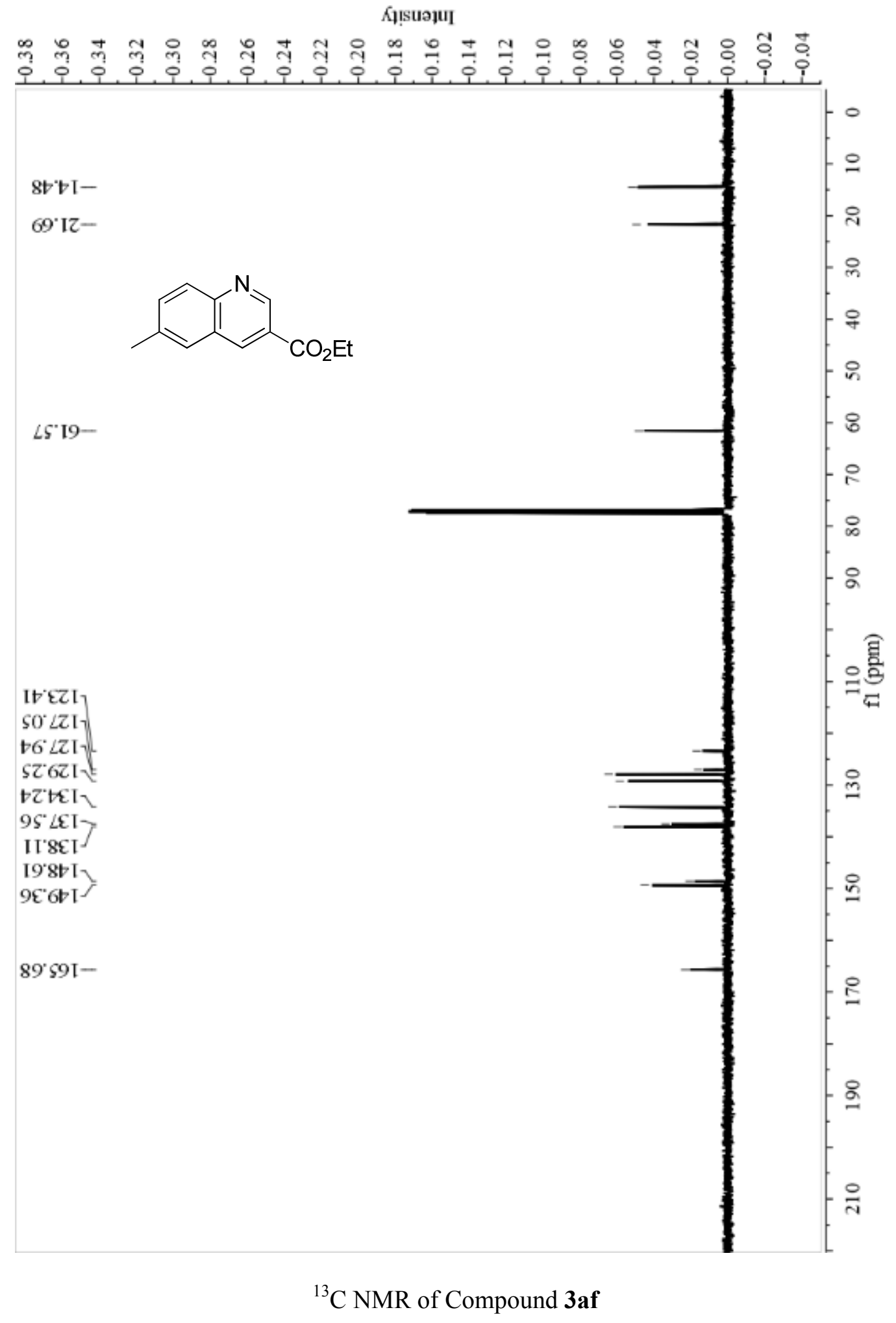




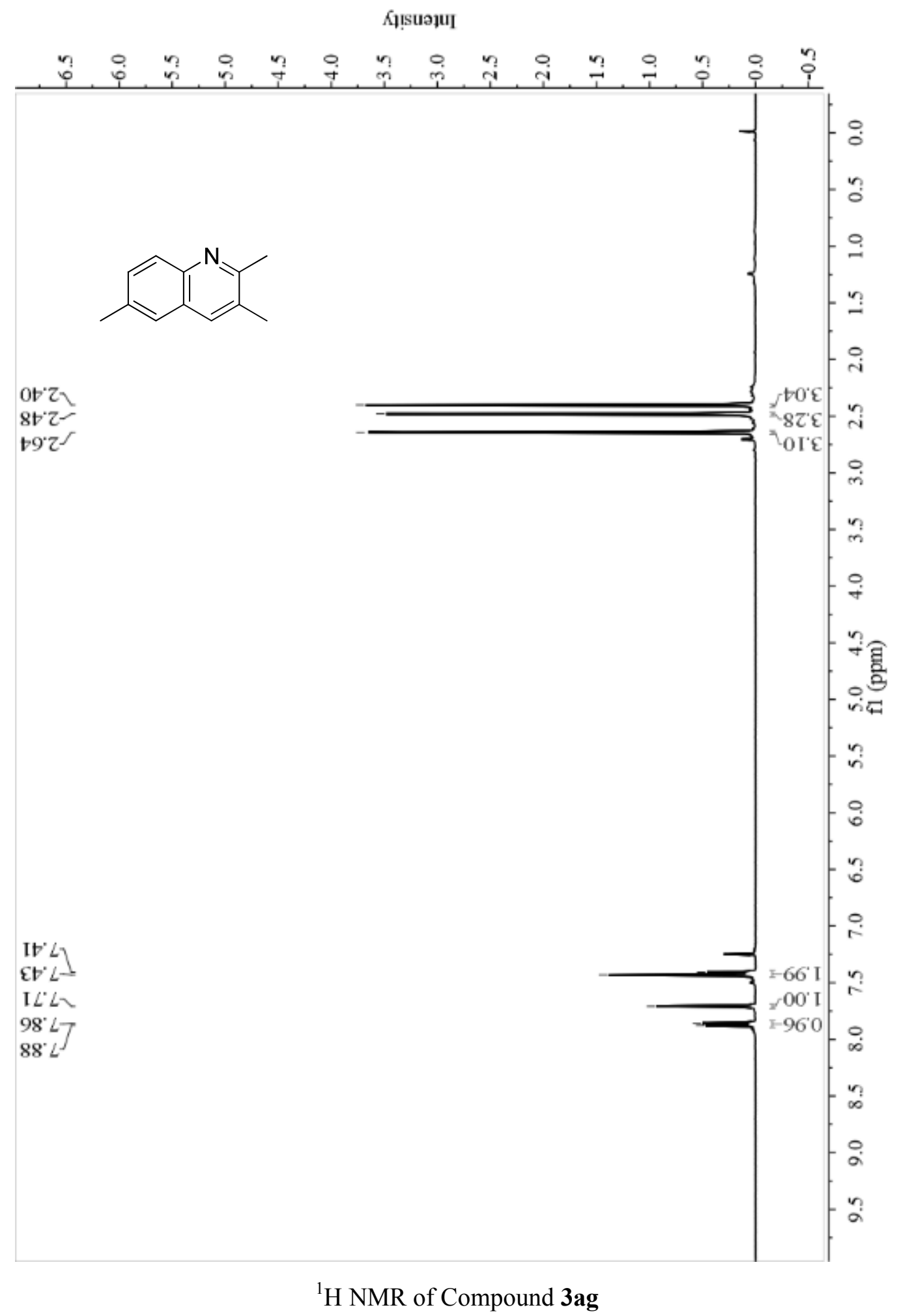




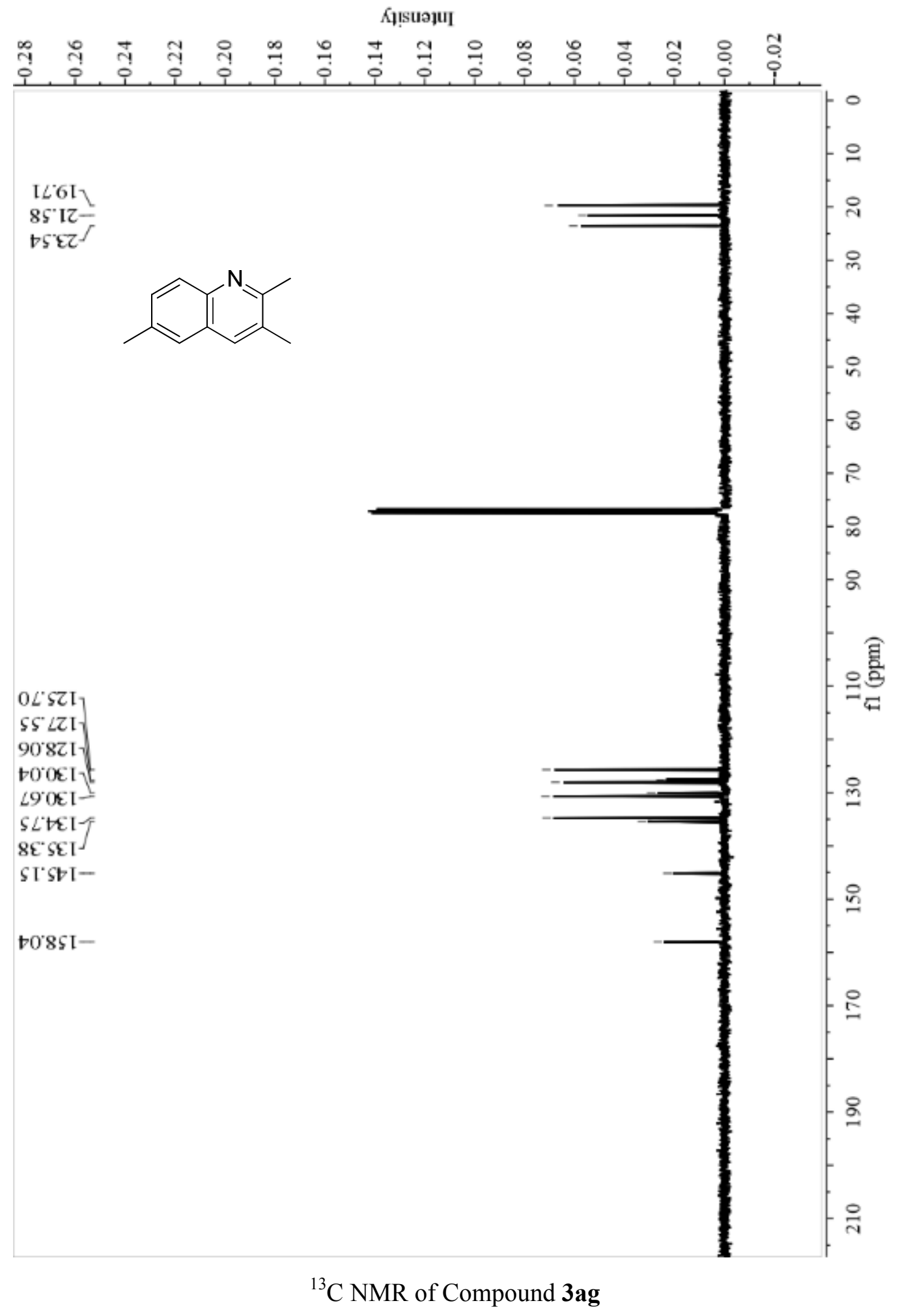




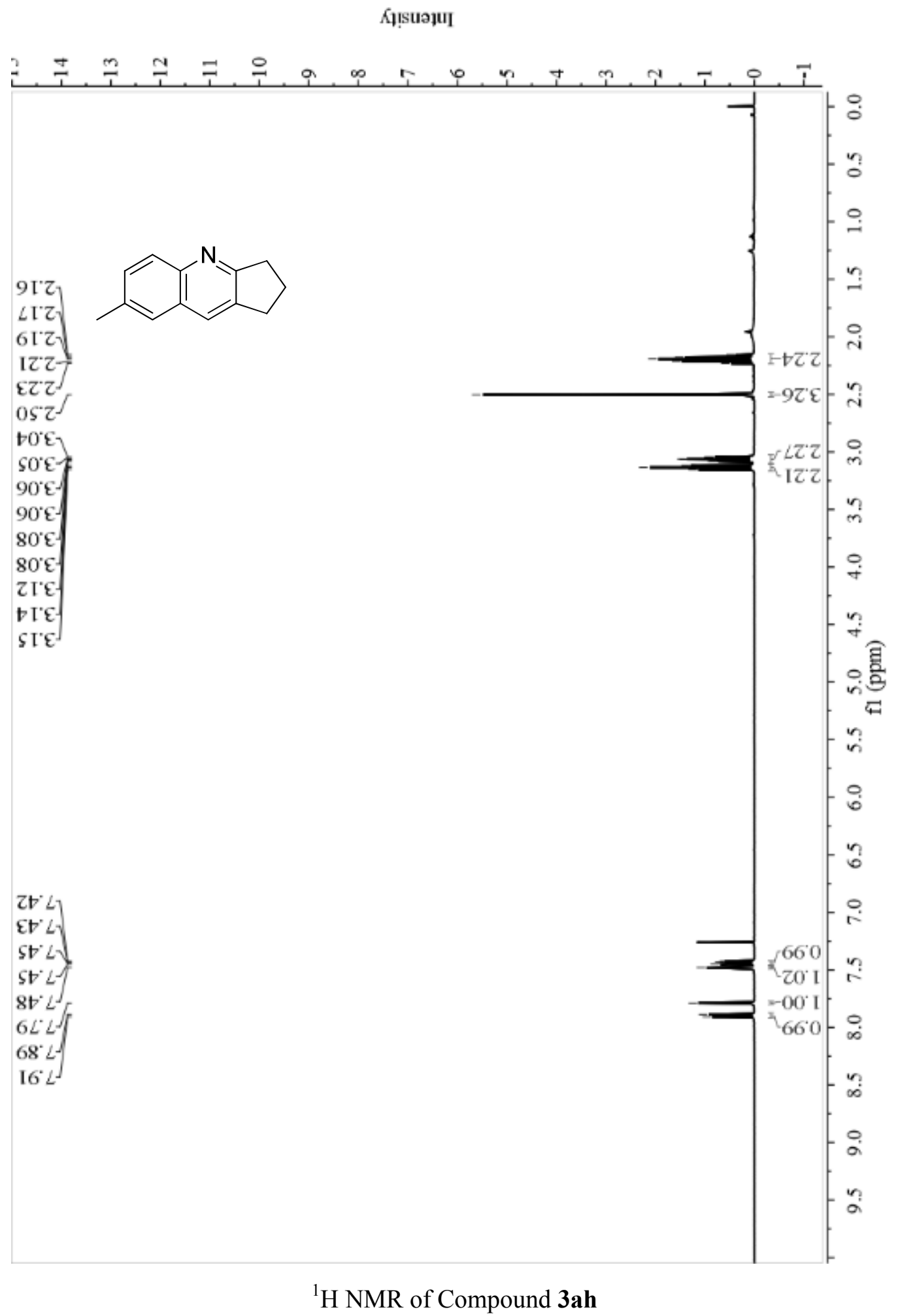




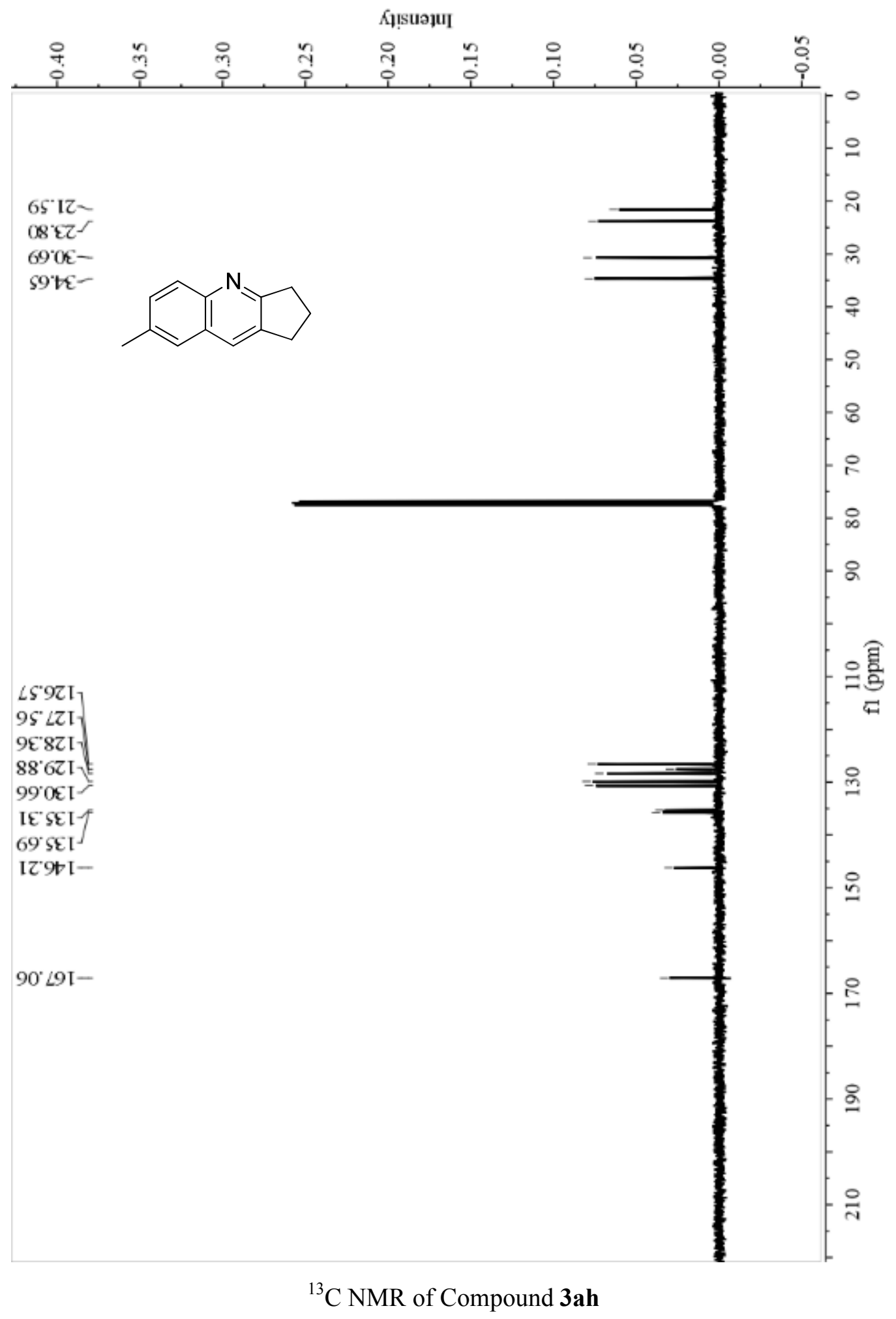




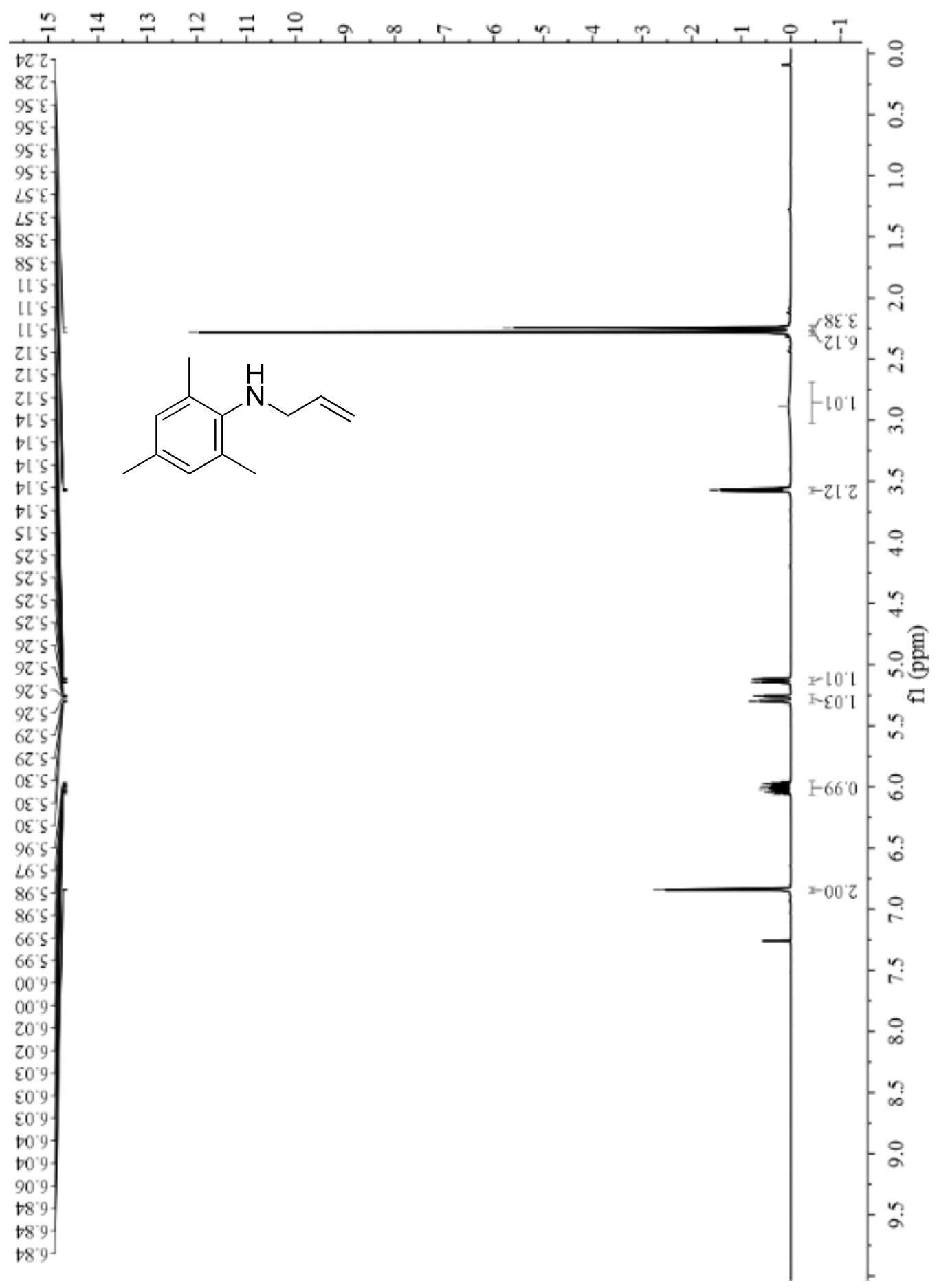

${ }^{1} \mathrm{H}$ NMR of Compound 5oa 


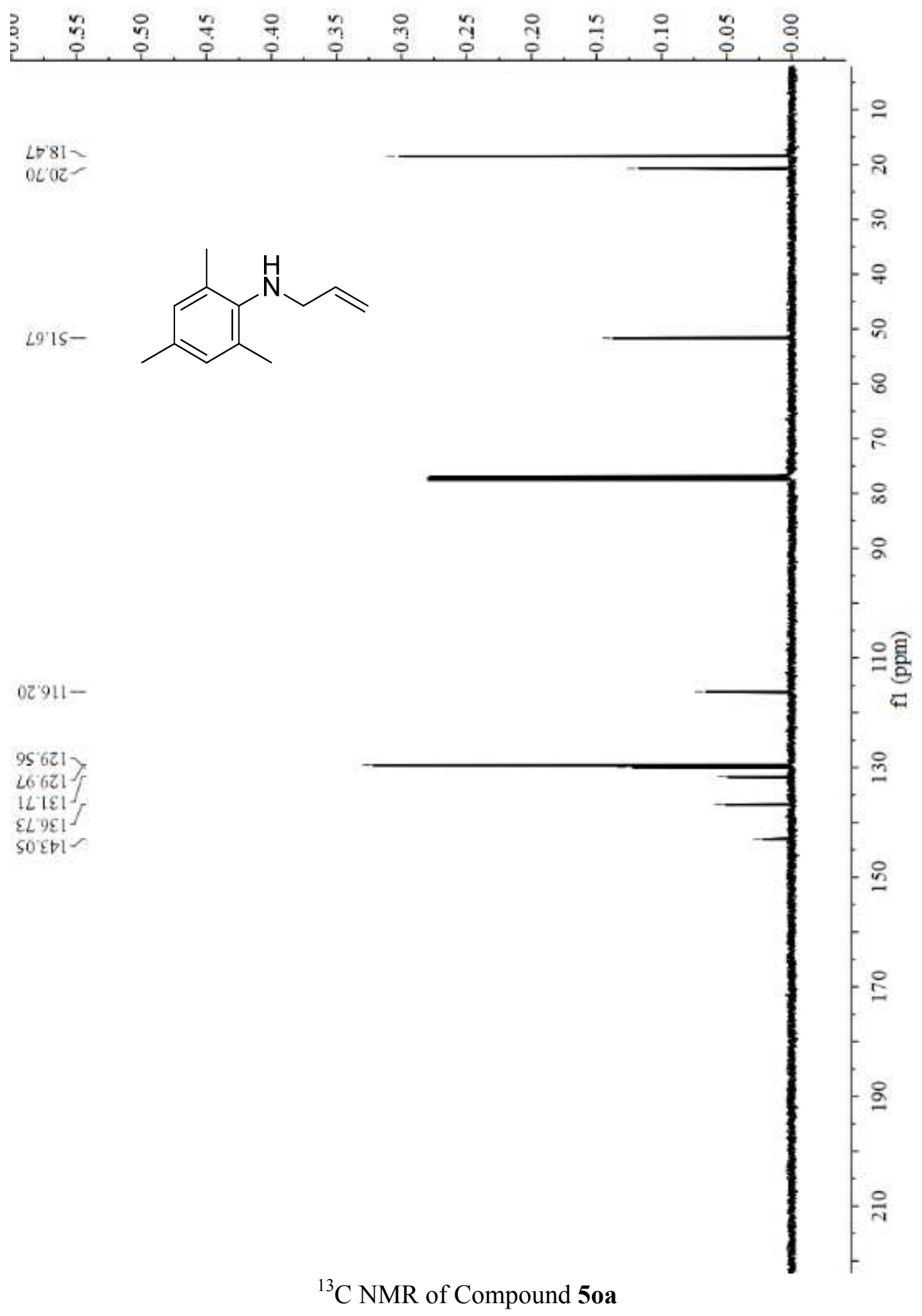

